\title{
Research for Electric Energy Systems - An Annual Report
}

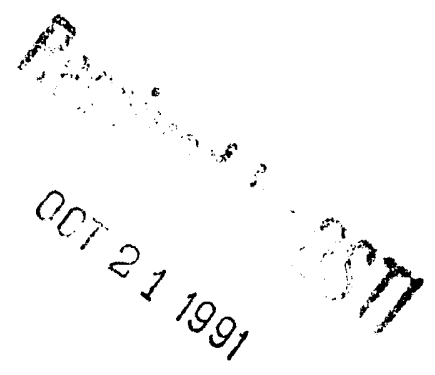

\section{William E. Anderson Editor}

U.S. DEPARTMENTI OF COMMERCE National Institute of Standards and Technology

Electronics and Electrical Engineering Laboratory

Electricity Division

Gaitheraburs, MD 20899

\section{Prepared for}

Department of Enersy

Division of Electric Energy Systems 1000 independence Avenue, SW Washington, DC 20585 


\title{
Research for Electric Energy Systems - An Annual Report
}

\section{William E. Anderson Editor}

\author{
U.S. DEPARTMENT OF COMMERCE \\ National Institute of Standards \\ and Technolody \\ Electronics and Electrical Engineering \\ Laboratory \\ Caitheraburg, MD 20899 \\ Prepared for \\ Department of Energy \\ Dlvision of Electric Energy Systems \\ 1000 Independence Avenue, SW \\ Washington, DC 20585
}

December 1990

lssued June 1991

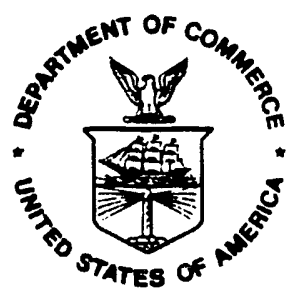

U.S. DEPARTMENT OF COMMERCE Robert A. Mosbacher, Secretary MATIONAL INSTITUTE OF STANDARDS AND TECHNOLOQY

lets w. Lyons, Rireste: 
This report summarizes the progress of four technical investigations conducted during CY 90. Although reasonable efforts have been made to ensure the reliability of the data presented, it must be emphasized that this is an interim progress report and that further experimentation and analysis may be performed before the conclusions from any of these investigations are formally published. It is therefore possible that some of the observations presented in this report will be modified, expanded, or clarified by our subsequent research ${ }^{1}$.

\footnotetext{
${ }^{1}$ The identification of commercial materials and their sources is made to describe the experiment adequately. In no case does this identification imply recommendation by the National Institute of Standards and Technology, nor does it imply that the product is the best available.
} 


\section{Table of Contents}

i ELECTRIC FIELD MEASUREMENTS 1

1.1 Introduction . . . . . . . . . . . . . . . . 1

1.2 Examination of Experimental Designs for In Vitro Studies Using ELF

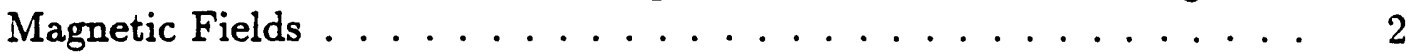

1.3 Experimental Geometry and Exposure Parameters . . . . . . . . . 2

1.3.1 Rectangular Geometry .................. 4

1.3.2 Cylindrical Geometry: Magnetic Field Perpendicular to Axis . 6

1.3.3 Cylindrical Geometry: Magnetic Field Parallel to Axis . . . . 8

1.4 Magnetic Fields from Parallel Plates . . . . . . . . . . . . . 11

2 GASEOUS DIELECTRICS RESEARCH 16

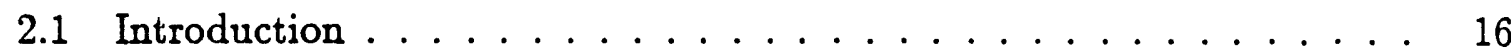

2.2 Detection of Trace $\mathrm{S}_{2} \mathrm{~F}_{10}$ in $\mathrm{SF}_{6} \ldots \ldots \ldots \ldots 17$

2.2 .1 Measurement System . . . . . . . . . . . . . . . 18

2.2.2 Data Acquisition and Analysis ............. . . 19

2.2.3 GC-MS Results and Calibration . . . . . . . . . . . . 20

2.2.4 Mass-Spectrometric Investigation of $\mathrm{S}_{2} \mathrm{~F}_{10}$ Pyrolysis . . . . . . 24

2.2.5 Gas-phase Impurities . . . . . . . . . . . . . . . . 30

2.2.6 Reference Sample Stability . . . . . . . . . . . . . 31

2.3 Stochastic Properties of Pulsating Partial Discharges . . . . . . . 33

2.3.1 Basic Considerations ................ 33

2.3.2 Measurement System . . . . . . . . . . . . . . 37

2.3.3 Examples of Results from Investigation of Trichel Pulses . . . 39

2.3.4 Influence of a Dielectric Barrier on the Stochastic Behavior of Trichel-Pulse Corona . . . . . . . . . . . . . 46

2.3.5 Limitations and Extensions of the SAPP Technique . . . . . 58

3 LIQUID DIELECTRICS RESEARCH 60

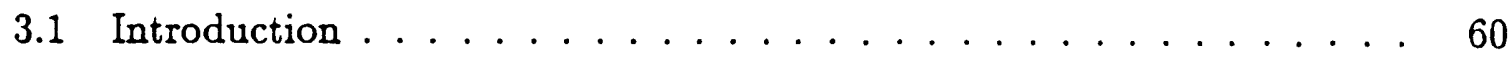

3.2 Streamer Initiation in Hexanes . . . . . . . . . . . . . . 61

3.2.1 Apparatus and Procedure ............. 61

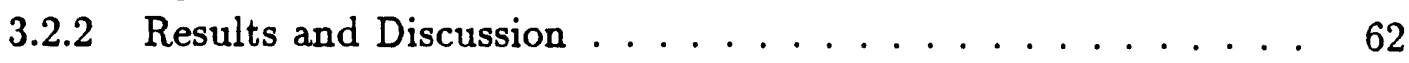

3.3 DC Voltage Conditions . . . . . . . . . . . . . . . 69

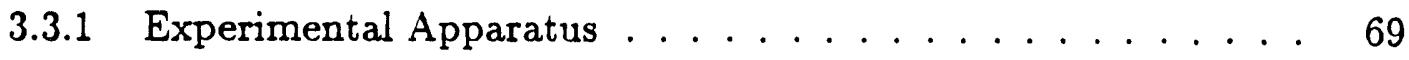

3.3.2 Partial Discharge Records . . . . . . . . . . . 69

3.4 Conclusions .......................... 72

4 FAST TRANSIENT MEASUREMENTS 73

4.1 Introduction . . . . . . . . . . . . . . 73 
4.2 Resistive Voltage Divider Design . . . . . . . . . . . . . it

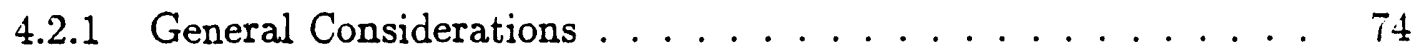

4.2.2 Configuration and Construction ............ 75

4.3 The Unit Step Response (USR) and Its Application . . . . . . . . . . 80

4.3.1 Step Response Measurement . . . . . . . . . . . . . 80

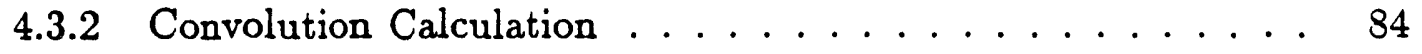

4.4 Conclusion . . . . . . . . . . . . . . . . 88 


\title{
Research for Electric Energy Systems - An Annual Report
}

\author{
William E. Anderson, Editor
}

\begin{abstract}
This report documents the technical progress in the four investigations which make up the project "Support of Research Projects for Electrical Energy Systems", Department of Energy Task Order Number 137, funded by the U.S. Department of Energy and performed by the Electricity Division of the National Institute of Standards and Technology (NIST). The first investigation is concerned with the measurement of magnetic fields in support of epidemiogical and in vitro studies of biological field effects. NIST cohosted a workshop on exposure and biological parameters that should be considered during in vitro studies with extremely low frequency (ELF) magnetic and electric fields. Additionally, equations were developed to predict the magnetic field in a parallel plate magnetic field exposure system. An IEEE standard prepared at NIST on measuring dc electric fields and ion related parameters was approved and published. Various site visits were made to characterize the electric and magnetic fields in biological exposure systems. The second investigation is concerned with two different activities: the detection of trace levels of $S_{2} F_{10}$ in $\mathrm{SF}_{6}$ and the development of an improved stochastic analyzer for pulsating phenomena (SAPP). The detection of $\mathrm{S}_{2} \mathrm{~F}_{10}$ in the presence of $\mathrm{SF}_{6}$ using massspectrometric detection coupled to a gas chromatograph is difficult because of the similar mass spectra. Enrichment techniques, capable of sub parts per billion ( $\mathrm{ppb}$ ) detection in the case where the background gas is not $\mathrm{SF}_{6}$, are unsuitable for this application. A technique is described that enables the detection of $\mathrm{S}_{2} \mathrm{~F}_{10}$ in gaseous $\mathrm{SF}_{6}$ down to the $\mathrm{ppb}$ level using a modified gas chromatograph-mass spectrometer. The SAPP has been improved to allow direct real-time measurements of conditional pulse-amplitude distributions of higher order than was previously possible. The new system was applied to an investigation of the stochastic behavior of negative corona (Trichel pulses) and the effect of a dielectric barrier on these discharges. The third investigation is concerned with breakdown and prebreakdown phenomena in liquid dielectrics. The activity reported here was a study of negative streamers preceding electric breakdown in hexanes. Using the image preserving optical delay, the growth of the streamers associated with partial discharges at a point cathode are photographed at high magnification. Simultaneous discharge current measurements enables a detailed description of the temporal and spatial
\end{abstract}


development of streamers and provide a basis for the evaluation of nodels for the initiation of negative streamers. The last investigation is concerned with the evaluation and improvement of methods for measuring fast transients in electrical power systems such as might be associated with an electromagnetic impulse. The new draft of IEC-60 recommends the use of an independent reference measurement system to verify the performance of the impulse voltage measuring system under test. A compact resistive divider, NIST4, was designed for this purpose. It is anticipated that this divider together with some Kerr electro-optical devices will be used as the reference system at NIST. The design details of NIST4 and its measurement capabilities are presented. 


\title{
1 ELECTRIC FIELD MEASUREMENTS
}

\author{
Task 01 \\ Martin Misakian \\ Electricity Division \\ National Institute of Standards and Technology
}

\subsection{Introduction}

The objectives of this project are to develop methods to evaluate and calibrate instruments which are used, or are being developed, to characterize the electrical parameters in the vicinity of power lines and in laboratory apparatus designed to simulate the power line environment. Electrical measurement support is also provided for the Department of Energy funded efforts to determine if there are biological effects due to ac fields and dc fields with ions.

During 1990, NIST organized and cohosted with Battelle Pacific Northwest Laboratories (BPNL) a workshop on exposure and biological parameters that should be considered during in vitro studies with extremely low frequency (ELF) magnetic and electric fields. The workshop was an outgrowth of discussions with BPNL following an NIST conference presentation late in 1989. It was evident at that time that all of the relevant exposure parameters during many in vitro bioeffect studies with ELF magnetic fields were not being adequately characterized. The NIST contribution to the workshop considered the electric field and current density distributions induced in culture medium. A portion of the workshop presentation is reproduced below. Also a related NIST paper describing an optimum experimental configuration for in vitro studies with ELF magnetic fields was published [1].

In addition efforts were also made to develop equations that would predict the magnetic field in a parallel plate magnetic field exposure system similar in principle to one developed at BPNL [2]. The results of the calculations are described below.

Other events and activities related to the NIST Fields Project during 1990 included (a) the approval and publication of an IEEE standard which provides guidance for measuring dc electric fields and ion related parameters (IEEE Std 1227-1990, IEEE Guide for the Measurement of DC Electric-Field Strength and Ion Related Quantities; draft standard prepared at NIST in collaboration with members of IEEE DC Fields and Ions Working Group), and (b) consultations with the Food and Drug Administration (electric and magnetic field measurements near electric blankets), the National Toxicology Program (experimental design and exposure parameters for rodent study with magnetic fields), and the Environmental Protection Agency (reviews of draft reports). NIST also coauthored two working group papers in the IEEE Power Engineering Society related to characterizing power line magnetic fields. 
Both papers are scheduled for publication in the IEEE Transactions on Power Delivery in early 1991. Site visits were made to Southwest Research Institute on behalf of DOE and to the University of Rochester and BPNL on behalf of EPRI, during which electric and magnetic fields were characterized in biological exposure systems and calibration apparatus. Preliminary measurements of power frequency magnetic fields in and near the NIST-Boulder daycare facility were performed. The measurements were coupled with model calculations to better characterize the sources of magnetic fields.

NIST serves on advisory panels for the California Department of Health Services and the National Cancer Institute (NCI), and review panels for the National Institutes of Health (NIH). During 1990, NIST participated in meetings of the NCI and NIH panels.

\subsection{Examination of Experimental Designs for In Vitro Studies Using ELF Magnetic Fields}

There have been many laboratory studies since the mid 1970's examining the possibility of biological effects to cells in vitro due to power frequency magnetic fields. These studies have typically used Helmholtz coils to produce an approximately uniform magnetic field for exposure purposes. Significantly, the enclosures containing the cells and liquid growth medium have varied in shape and size. Because the geometry of the liquid volume, which is determined by the enclosure, has a major impact on the exposure parameters, the enclosure must be considered as an important part of the exposure system. This portion of the report examines the exposure parameters for several experimental configurations that have been used during in vitro studies and notes some of their advantages and limitations. The numerous biological constraints that must be considered for the different configurations will be the subject of a future report. Extensive use is made of the equations developed by McLeod et al. [3] for determining the current density and electric field induced in the liquid growth medium by the magnetic field.

\subsection{Experimental Geometry and Exposure Parameters}

In vitro studies with power frequency magnetic fields have often been conducted using circular plastic or glass petri dishes and rectangularly shaped vessels as the enclosures. When the enclosure containing the liquid and biological cells is introduced into the magnetic field, an electric field and an associated current are induced in the liquid by the time-varying magnetic field. Thus, the cells are exposed to three candidate parameters that may individually, or in combination, cause a biological effect, i.e., the magnetic field or flux density, $B$, the induced electric field strength, $E$, and the induced current density, $J$. While all the cells will experience essentially the same 


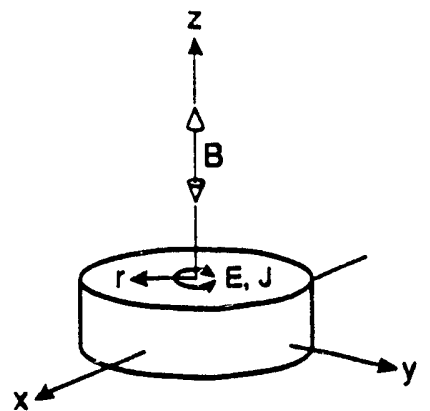

(a)

$$
\begin{aligned}
J & =\sigma \pi f \mathrm{Br} \\
E & =\pi f \mathrm{Br}
\end{aligned}
$$

B.R. McLeod ot al.

Bioelectromagnotics 4:357-370 (1983)
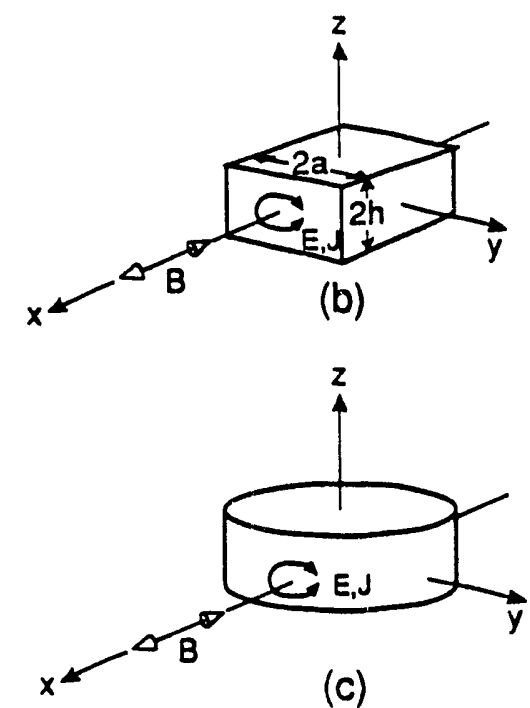

$$
\begin{aligned}
& J y \equiv i \frac{\omega \sigma B 2 a}{\pi^{2}}{ }_{n} \Sigma_{\text {odd }} \frac{4}{n^{2}}\left(\cos \left(\frac{n \pi}{2 a}\right) y\right)\left(\frac{\sinh \frac{n \pi}{2 a} z}{\cosh \frac{n \pi}{2 a} h}\right) \\
& J z \cong-i \frac{\omega \sigma B 2 h}{\pi^{2}} \Sigma_{\text {odd }} \frac{4}{m^{2}}\left({ }^{ \pm} \cos \left(\frac{m \pi}{2 h}\right) z\right)\left(\frac{\sinh \frac{m \pi}{2 h} y}{\cosh \frac{m \pi}{2 h} a}\right)
\end{aligned}
$$

B.R. McLeod ot al. Bioelectromagnotićs 4:357-370 (1983)

Figure 1. Experimental configurations for in vitro studies with ELF magnetic fields.

magnetic field, the exposure to $E$ and $J$ will depend on the size and shape of the liquid volume, the direction of the magnetic field with respect to the liquid volume, and the location of the cell in the liquid volume.

We consider the three experimental configurations shown in figure 1a: a cylindrical liquid volume with the power frequency magnetic field parallel and perpendicular to the cylinder axis (figure $1 \mathrm{a}$ and $1 c$ ) and a rectangular liquid volume with the magnetic field perpendicular to one of the faces (figure 1b). It should be noted that the direction and magnitude of any dc magnetic field may be of significance if tests of a resonance model are being conducted. However such experiments are not considered here. As qualitatively indicated in figure 1, the induced electric field lines and current paths are circular when the magnetic field is parallel to the cylinder axis. In general, the electric field lines and current paths form noncircular loops for the remaining two cases. The equations for the induced current density and electric field are simplest when the magnetic field is parallel to the cylinder axis. Then the current density is given by [3] 


$$
J=\sigma \pi f B r
$$

where $\sigma$ is the conductivity, $f$ is the frequency and $r$ is the radial distance from the cylinder axis. Because the current density is equal to the product $\sigma E$, the electric field strength is just

$$
E=J / \sigma=\pi f B r
$$

The expressions for the induced current density in the rectangular volume are more complicated and are given by [1]

$$
J_{y} \simeq i \frac{\omega \sigma B 2 a}{\pi^{2}} \sum_{\substack{n \\ \text { odd }}} \frac{4}{n^{2}}\left( \pm \cos \frac{n \pi y}{2 a}\right)\left(\frac{\sinh \frac{n \pi}{2 a} z}{\cosh \frac{n \pi}{2 a} h}\right)
$$

and

$$
J_{z} \simeq-i \frac{\omega \sigma B 2 h}{\pi^{2}} \sum_{\substack{m \\ \text { odd }}} \frac{4}{m^{2}}\left( \pm \cos \frac{m \pi z}{2 h}\right)\left(\frac{\sinh \frac{m \pi}{2 h} y}{\cosh \frac{m \pi}{2 h} a}\right)
$$

where $2 a$ is the width of the liquid volume, $2 h$ is the depth, and $\omega$ is $2 \pi$ times the frequency, $f$. The \pm sign indicates that the terms in the summation are alternatively added and subtracted. As above, the corresponding expressions for the electric field are found by dividing equations (3) and (4) by the conductivity, $\sigma$. As shown by McLeod et al. [3], the case of the cylindrical volume with the magnetic field perpendicular to the cylinder axis can be treated by cutting the cylinder into approximately rectangular volumes as shown in figure $1 \mathrm{c}$ and using equations (3) and (4) for each rectangular volume. The rectangular geometry is examined first.

\subsubsection{Rectangular Geometry}

With the rectangular configuraticn shown in figure $1 \mathrm{~b}$, it can be shown, using equations (3) and (4), that the induced current and electric field vanish along the $\mathrm{x}$-axis as well as along the four edges parallel to the $\mathrm{x}$-axis. The maximum values of the induced electric field and current density occur at the liquid boundary but the locations depend on the cross sectional shape of the volume. The cells will all experience the same magnetic field because the magnetic field is uniform throughout the liquid volume. However, the electric field and current density experienced by the cells will vary from zero to some maximum value depending on the location of the 


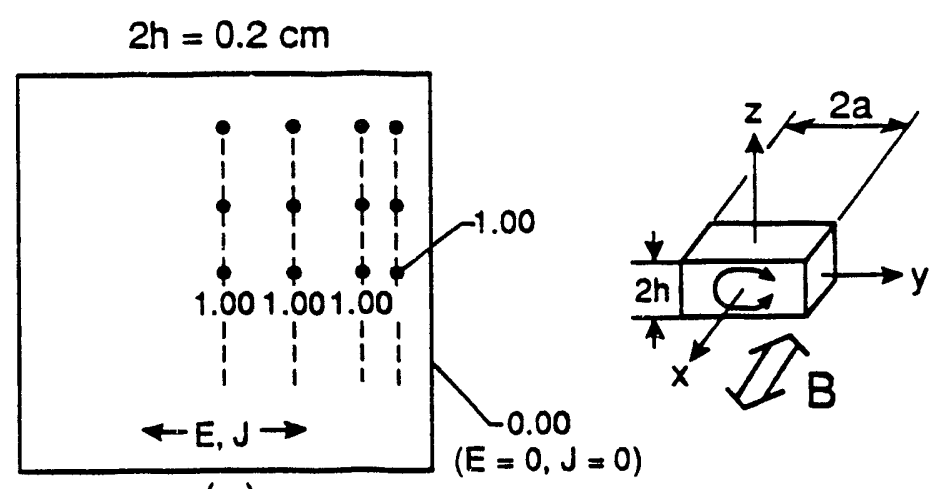

(a)

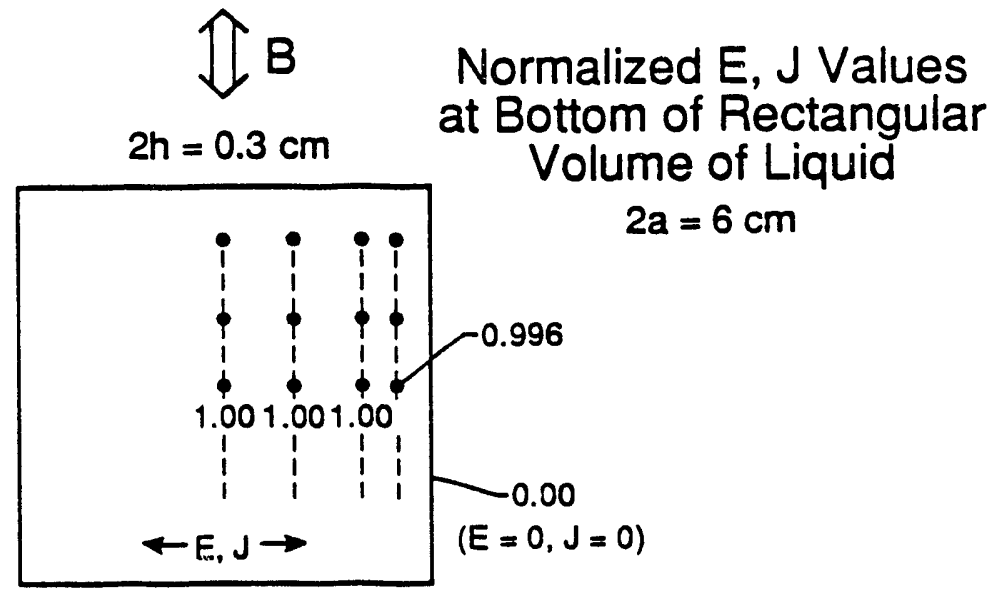

(b)

Figure 2. Normalized values and directions of $E$ or $J$ along bottom surface of culture medium when $B$ is parallel to $\mathrm{x}$-axis (a) culture medium is $2 \mathrm{~mm}$ deep (b) culture medium is $3 \mathrm{~mm}$ deep.

cell. If the approximate region the cells occupied during exposure to the magnetic field were known, their exposure to a more limited range of $E$ and $J$ values could be determined using equations (3) and (4). Such information is, in general, not known when experiments are conducted with cells in suspension.

Our ability to describe the exposure conditions for the cells improves if the cells can be cultured on the bottom surface of the enclosure. Then the vertical components of $E$ and $J$ vanish and approximately unifo:m values of $E$ and $J$ in the horizontal direction can be produced along the bottom surface of the liquid if the liquid depth is small compared to the width, i.e., $2 h \ll 2 a$. Figure $2 \mathrm{a}$ shows normalized values of $E$ or $J$ calculated at the bottom surface of the liquid volume for $2 a=6 \mathrm{~cm}$ and $2 h=0.2 \mathrm{~cm}$. It can be seen for this example that the region of approximately uniform $E$ or $J$ extends almost to the side edges where $E$ and $J$ rapidly decrease to zero. Increasing the depth of the licuid by $1 \mathrm{~mm}$ decreases the uniformity, but because the liquid depth, $2 h$, is still much smaller than the side dimension, $2 a$, the 
uniformity is still very good as shown in figure $2 \mathrm{~b}$. While the $1 \mathrm{~mm}$ difference in depth has had a small effect on the uniformity of $E$ and $J$ in this example, the increase in magnitude of $E$ and $J$ is not insignificant, amounting to $50 \%$ over most of the area. This sensitivity of $E$ and $J$ to liquid depth for the rectangular configuration may be an important consideration when attempts are made to replicate the results of an earlier in vitro study.

It should also be noted that cells cultured on the bottom surface of the enclosure will experience an "uneven" exposure to the approximately uniform $E$ and $J$ values described above because a portion of the underside of the cell, which is in contact with plastic or glass, will not be exposed to the electric field or current density in the liquid. This problem can be reduced if the cells can be cultured in agar that has the same conductivity as the liquid. However, the use of agar can introduce undesirable biological effects [4] and cause significant changes in the exposure conditions. For example, if liquid $2 \mathrm{~mm}$ deep covers a layer of agar $2 \mathrm{~mm}$ thick on the bottom of a rer,tangular enclosure $3 \mathrm{~cm}$ wide, and if cells are cultured near the upper surface of the agar, they will experience little or no exposure to $E$ or $J$ except near the side edges.

Summarizing the above discussion, the rectangular configuration allows for the exposure of large numbers of cells to uniform values of $B, E$, and $J$ if they are cultured on the bottom surface of the enclosure. The values of $E$ and $J$ are sensitive to liquid depth and can be calculated using equations (3) and (4). The equations for determining $E$ and $J$ are not elementary, but can be readily evaluated with a computer. The use of agar can significantly influence the exposure parameters can and also have biological consequences.

\subsubsection{Cylindrical Geometry: Magnetic Field Perpendicular to Axis}

As noted earlier, the case of a cylindrical liquid volume with the magnetic field perpendicular to the cylindrical axis can be treated by imagining the cylinder being cut into rectangular sections and using the theory for rectangular geometry. Thus, the above remarks regarding the characterization of exposure conditions for cells in suspension and cells cultured on the bottom of the enclosure again apply to each section. Figure 3 shows normalized values of $E$ and $J$ at the bottom surface of a liquid volume $6 \mathrm{~cm}$ in diameter and $0.2 \mathrm{~cm}$ deep. The uniformity is again seen to be very good over most of the surface area and is a consequence of the fact that $2 h$ is still much smaller than the width of the imaginary sections at most locations. As for the examples considered in figure 2, the magnitude of $E$ or $J$ is sensitive to the liquid depth. 


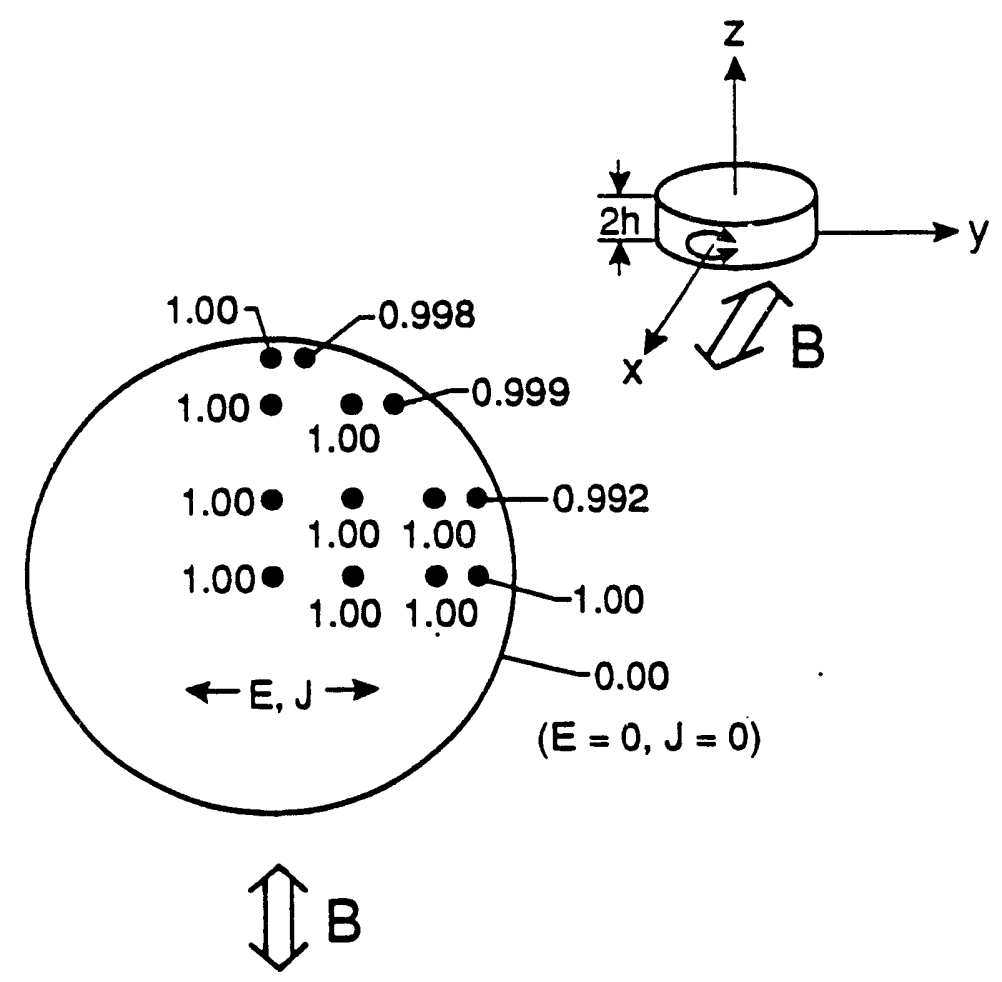

Normalized E, J Values at Bottom of Cylindrical Volume of Liquid

$$
\begin{gathered}
B \perp \text { to axis } \\
\text { diameter }=6 \mathrm{~cm} \\
2 \mathrm{~h}=0.2 \mathrm{~cm}
\end{gathered}
$$

Figure 3. Normalized values and directions of $E$ and $J$ along bottom surface of culture medium when $B$ is parallel to $\mathrm{x}$-axis. 

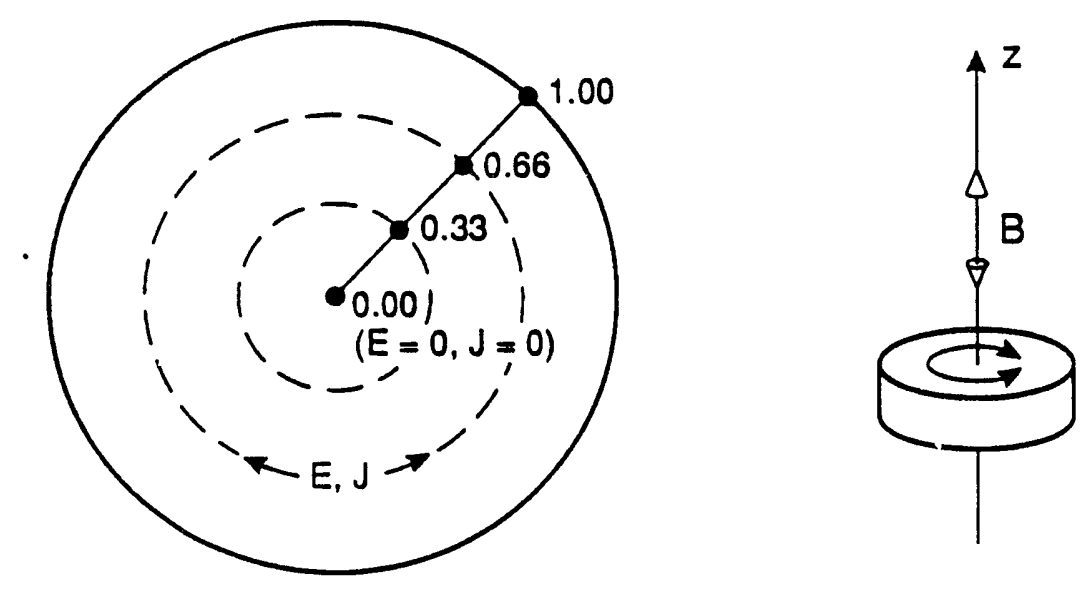

\title{
Normalized E, J Values in Cylindrical Volume of Liquid
}

\author{
diameter $=6 \mathrm{~cm}$ \\ B II to cylinder axis
}

Figure 4. Normalized values and directions of $E$ and $J$ in culture medium when $B$ is parallel to $z$-axis

\subsubsection{Cylindrical Geometry: Magnetic Field Parallel to Axis}

When the magnetic field is parallel to a cylindrical volume, the problem of describing the exposure conditions for the cells becomes more simple. The equations describing $E$ and $J$ (equations(1) and (2)) are elementary and indicate that both $E$ and $J$ increase linearly with radial distance from the cylinder axis. As noted earlier, the electric field lines and current density form concentric circular loops and are thus more easily visualized than for the rectangular geometry. Significantly, equations (1) and (2) indicate that $E$ and $J$ are independent of liquid or agar depth.

Figure 4 shows normalized $E$ and $J$ values for a cylindrical volume $6 \mathrm{~cm}$ in diameter. While the lack of uniformity of these exposure parameters may at first seem to be a disadvantage when characterizing cell exposure, it is possible to make use of the nonuniformity to obtain several ranges of exposure to $E$ and $J$ during the same experimental run [1]. For example, cells can be segregated into annular sections with circular walls and the range of $E$ and $J$ values experienced by the cells in each section can be determined from equations (1) and (2) and the radii that define the section. The exposure geometry and design of an enclosure that would be suitable for short term studies with cells in suspension are shown in figure 5.

While the cylindrical volume in figure 5 has been partitioned into th.ee sections, more or fewer sections could be used. For more extended periods of exposure, lasting as 

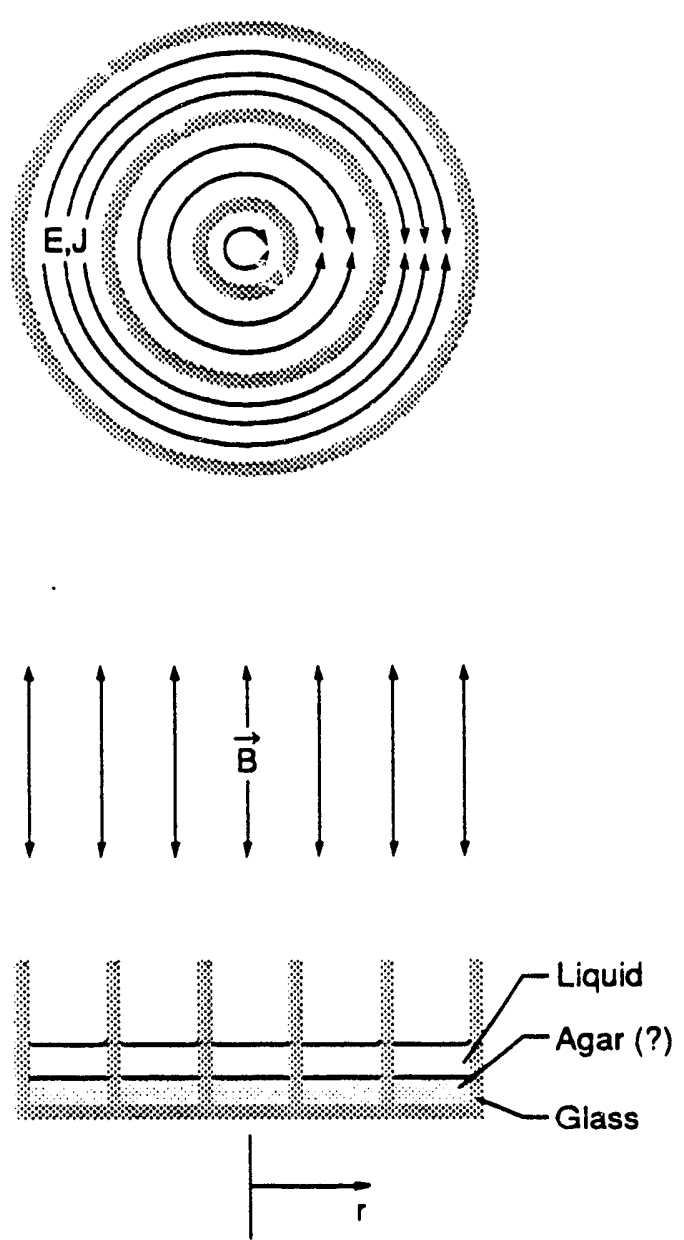

Figure 5. Experimental design for short term in vitro studies with cells in suspension. 


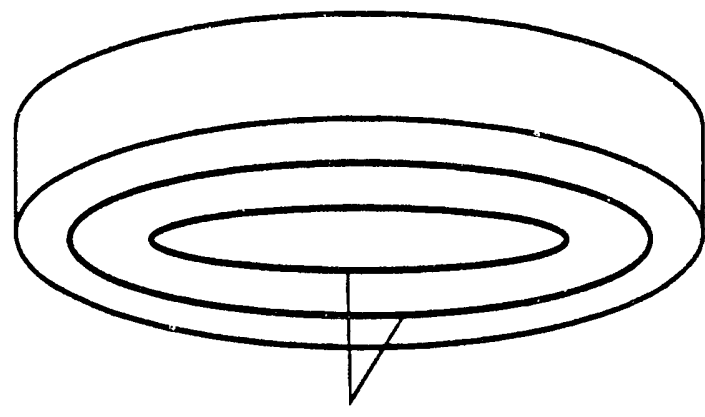

Annular regions indicated with circles painted on bottom of petri dish

Figure 6. Method for characterizing radial positions of cells.

long as six or seven days, the enclosure can be fitted with a cap and "sealed" with commercially available laboratory film which prevents significant evaporation but allows adequate air exchange [5]. Vertical stacking of the enclosures is one technique for increasing the number of cells that are exposed during a single experimental run. Introducing a layer of agar at the bottom of the enclosure as shown in figure 5 provides for a more uniform exposure of the cells (top and underside) to $E$ and $J$. Unlike the rectangular geometry case, introduction of the agar will not affect the values of $E$ and $J$ because $E$ and $J$ are independent of liquid or agar depth. However, use $c^{f}$ agar can have siguificant biological effects as noted previously.

If the circular walls shown in figure 5 introduce an impediment for cells plated directly on the enclosure surface, it may be possible to replace the walls with painted concentric circles on the underside of the enclosure as shown in figure 6 .

Other techniques for characterizing the radial positions of the cells in the cylindrical enclosure include growing the cells on a flass cover slip placed in the enclosure (the cover slip can be removed later for measurements with a rule and compass) or indicating the annular regions on a flat surface, as shown in figure 7 , upon which the enclosure is placed.

Because of the spatial uniformity of $B$ and the readily determined variation of $E$ and $J$ with position in the enclosure, the experimental configuration described in this section can be used to investigate directly questions regarding the relative significance of interaction mechanisms involving the magnetic field and/or induced electric field and current density. For example, the observation of a biological effect at the same level in all sections of the enclosure would suggest a mechanism involving direct magnetic interaction, whereas an effect that differed between sections could be indicative of a mechanism involving the induced electric field and magnetic field combination. For the latter case, a single experiment could provide sata for a range of $E$ or $J$ exposure levels. 


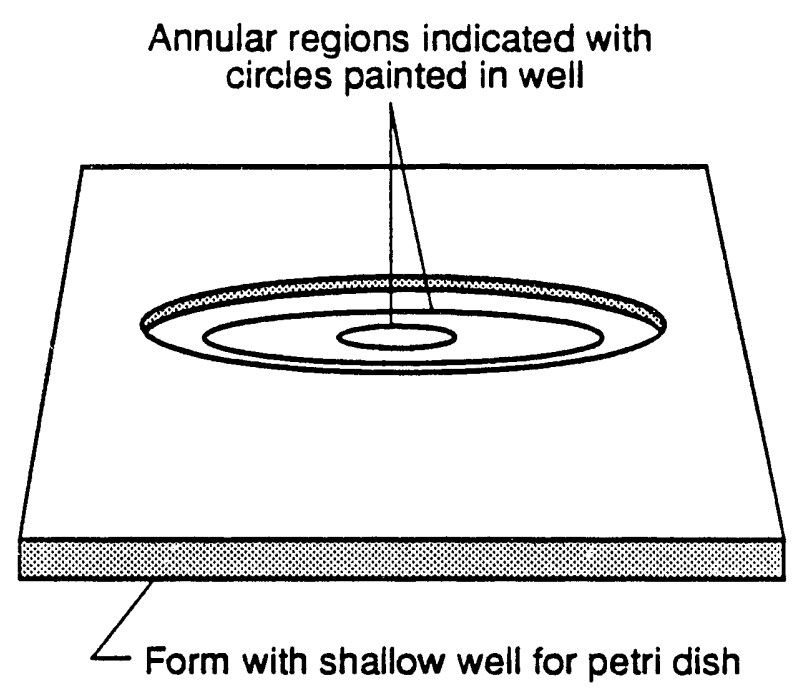

Figure 7. Method for characterizing radial positions of cells.

Summarizing this section, the cylindrical configuration with the magnetic field parallel to the cylinder axis results in induced electric fields and current densities that are easily visualized, and elementary equations for calculating $E$ and $J$ that are independent of liquid depth. In addition, the amount of information pertaining to dose-response during a single experimental run can be maximized, and it may be possible to distinguish between a purely magnetic field bioeffect and a bioeffect that involves the electric field or current density-magnetic field combination. The cunfiguration in this section does not permit exposure of large numbers of cells to the same $E$ and $J$ values. The use of agar does not have a significant effect on $E$ and $J$ values, but can have biological effects as noted earlier.

\subsection{Magnetic Fields from Parallel Plates}

Magnetic fields for ELF bioeffect studies have typically been produced with solenoids, Helmholtz coils, or rectangular coils with a common axis. Because stray fields from such coil systems can extend to considerable distances and because of the difficulty in shielding sham exposed animals or cells from these fields, it is of interest to consider an experimental apparatus recently reported by Miller et al. [2], that has low stray fields. Figure 8 shows a schematic view of the apparatus which consists, in part, of three stacked parallel plates. The ac current in the central plate divides equally between the upper and lower plates and produces a magnetic field parallel to the plates in each half of the apparatus as shown schematically in figure 8 at some instant. Because the fields in the two sections are in opposite directions, stray fields from the apparatus decrease more rapidly than the field from either section alone. This section describes attempts to theoretically describe the magnetic field for a similar parallel plate apparatus. 


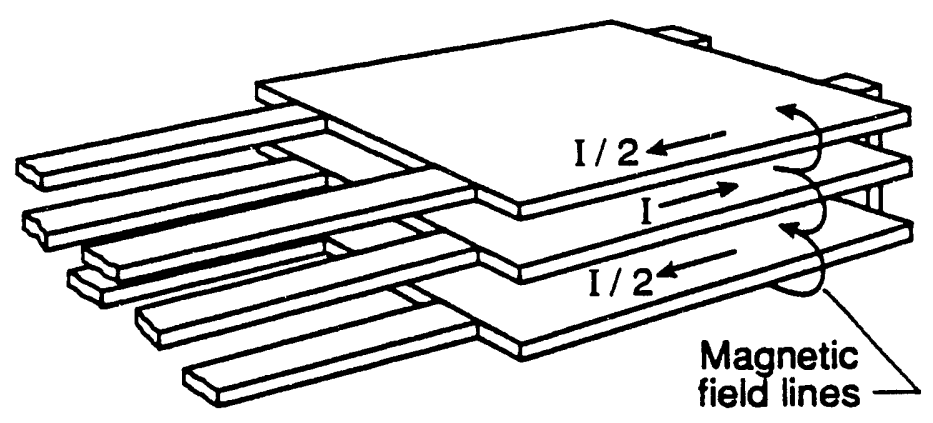

Figure 8. Schematic view of parallel plate apparatus (after Ref. 2) showing direction of ac current at some instant and direction of magnetic field between parallel plates.

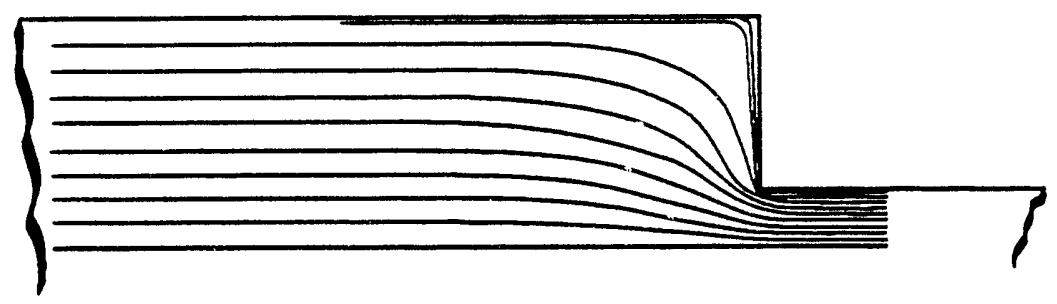

Figure 9. Half-section view of current distribution in flat conductor that changes in width by a factor of four.

Efforts to calculate the field for the apparatus shown in figure 8 are complicated by the nonuniformity of the current density at the points of connection between the plates and the electrodes that provide current. Figure 9 shows how the current distribution departs from uniformity when a single connecting electrode is one-fourth as wide as the plate [6].

The nonuniformity of the current density due to connections of metal of uneven widths can be overcome by the modification shown in figure 10 . The electrodes providing current to the plates are expanded in width to match the parallel plates and the shape has been changed to resemble two rectangular solenoids. Attempts were made to determine the magnetic field theoretically by applying the law of Biot-Savart to just the upper half of the arrangement as shown in figure 11. Because of the quasistatic nature of the field, it was assumed that the time dependence of the magnetic field

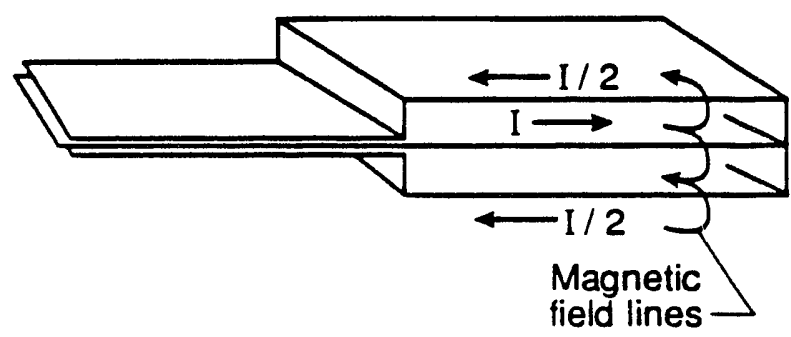

Figure 10. Modified parallel plate apparatus. 

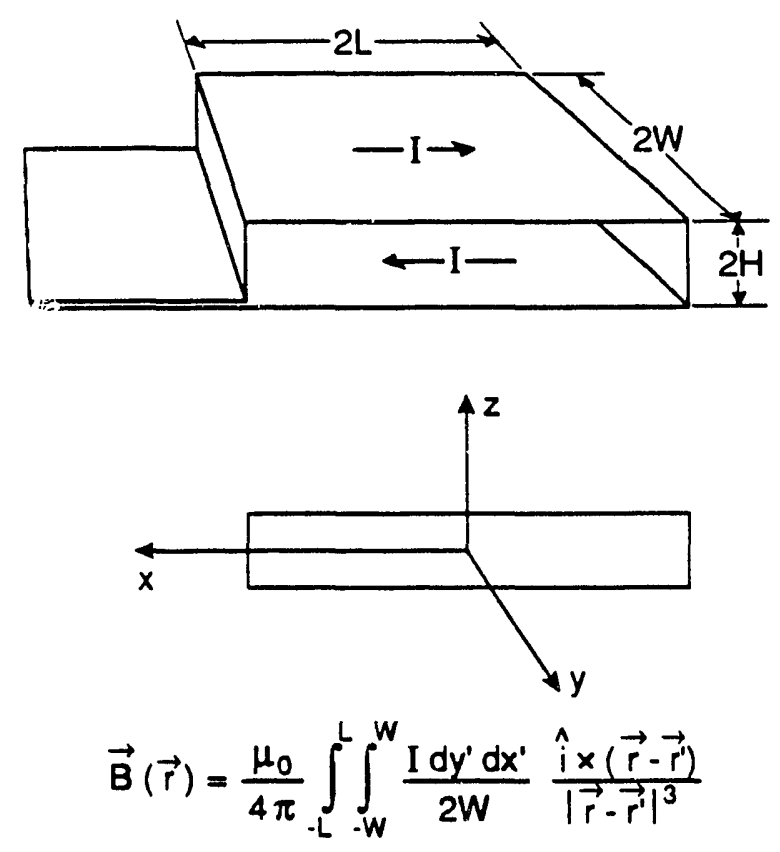

Figure 11. Geometry and coordinates for upper half of parallel plate exposure apparatus. The law of Biot-Savart takes the form of a double integral (example shown above) and is applied to all surfaces of the apparatus.

could be predicted by multiplying the dc magnetic field (obtained from the law of Biot-Savart) by a sin $\omega t$ term.

Figure 12 shows comparisons of measured horizontal magnetic field profiles at frequencies of $25 \mathrm{~Hz}$ and $1 \mathrm{kHz}$ in a rectangular apparatus as shown in figure 11 with dimensions of $50 \mathrm{~cm} \times 50 \mathrm{~cm} \times 10 \mathrm{~cm}$ side dimension. The measurements were performed with a miniature coil probe $5.7 \mathrm{~mm}$ long and $4.4 \mathrm{~mm}$ in diameter. Agreement is fair at $25 \mathrm{~Hz}$ and poor at $1 \mathrm{kHz}$. At $60 \mathrm{~Hz}$, the magnetic field is about $3.5 \%$ less than the dc value at the center of the apparatus. The departure from the dc theory is due to a skin effect [7] which causes the current to concentrate along the edges of the plate as shown schematically in figure 13.

The concentration results because of the larger inductance (and correspondingly increased impedance) along a central path through the plates. It is noted that even with nonuniform current distributions, regions of approximately uniform magnetic field can be obtained between the parallel plates with an apparatus similar to that shown in figure $8[2]$. The field magnitude and uniformity must be determined by measurement, however.

The problems with current nonuniformity (including the skin effect) can be overcome by replacing the metal plates with two adjacent rectangular solenoids as shown in figure 14 . 


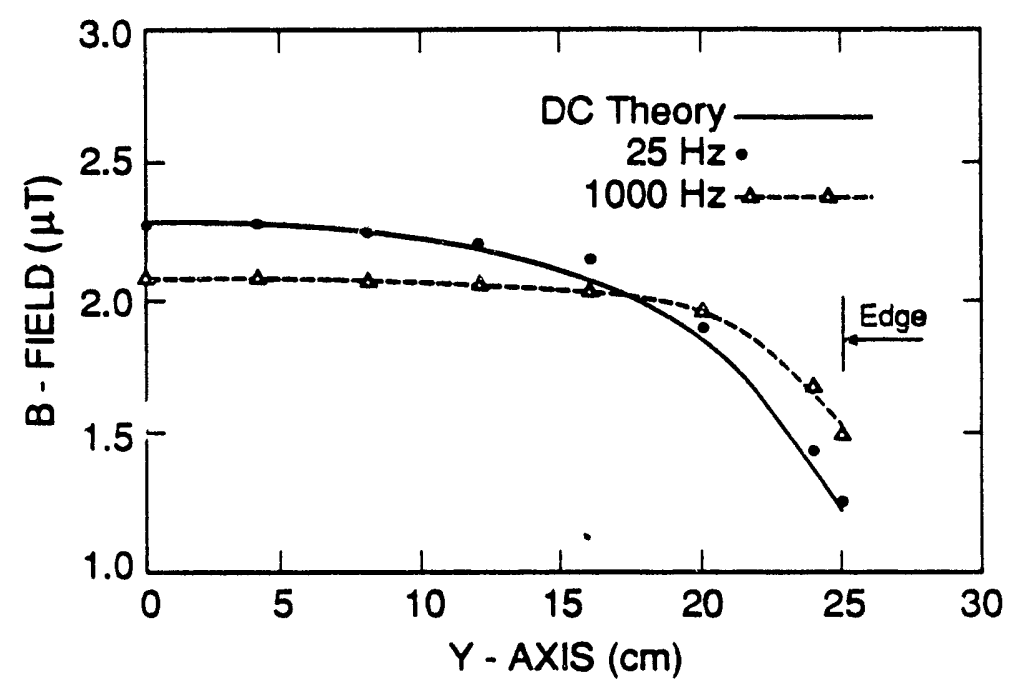

Figure 12. Horizontal magnetic field profiles midway between top and bottom plates of parallel plate apparatus $50 \mathrm{~cm} \times 50 \mathrm{~cm} \times 10 \mathrm{~cm}$ spacing. Magnetic field values for $\mathrm{dc}, 25 \mathrm{~Hz}$, and $1 \mathrm{kHz}$ frequencies are indicated.

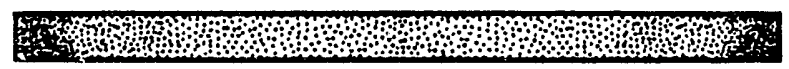

Figure 13. Edge-on view of plate showing increased current density concentration at edges of plate.

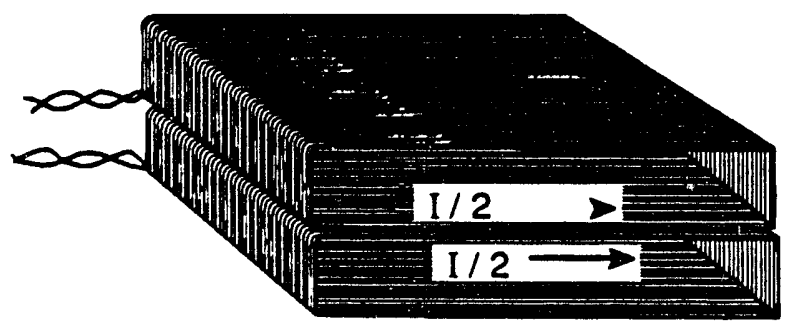

Figure 14. Arrangement of "coupled" rectangular solenoids to reduce stray magnetic fields. 
The fields in each section as well as the stray fields from the "coupled" arrangement can be calculated using equations for rectangular loops of wire [8] and the principle of superposition. The design shown in figure 14 eliminates significant skin effects and requires less current than the plates to produce the same field because of the many turns of wire. As for the parallel plate arrangement, the stray field from the "coupled" rectangular solenoids will decrease faster than either solenoid alone. 


\title{
2 GASEOUS DIELECTRICS RESEARCH
}

Task 02

\author{
Richard J. Van Brunt and James K. Olthoff \\ Electricity Division \\ John T. Herron \\ Chemical Kinetics Division \\ National Institute of Standards and Technology \\ Sanjay V. Kulkarni \\ Guest Scientist \\ Plasma Research Institute, India \\ Vishnu K. Lakdawala \\ Guest Scientist \\ Old Dominion University, Norfolk, VA
}

\subsection{Introduction}

The objectives of this project are the development of measurement capabilities and the providing of fundamental data as part of the Department of Energy's basic research concerned with the development and evaluation of advanced compressedgas-insulation technology.

To reduce space requirements and improve the efficiency of high-voltage transmission systems, the electric power industry has turned toward more extensive use of compressed-gas insulation. To design meaningful tests of system performance and establish specifications for the quality of materials used in such systems, more information is needed about the fundamental physical and chemical processes which lead to insulation deterioration and electrical breakdown. The research includes applications of gas chromatography-mass spectrometry to characterize corona discharge by-products; and the acquisition of fundamental data, such as, reaction-rate coefficients, corona-inception voltages, production rates of corona byproducts, the effects of contaminants on discharge initiation, and the rates of discharge-induced decomposition of the gas.

This section of the report highlights the progress of research in two areas. The first is concerned with the development of a sensitive method for detecting trace levels of disulfur decafluoride $\left(\mathrm{S}_{2} \mathrm{~F}_{10}\right)$ in sulfur hexafluoride $\left(\mathrm{SF}_{6}\right)$ by gas chromatography mass spectrometry. The second area of research has to do with development of an improved stochastic analyzer for pulsating phenomena (SAPP) and its application to the investigation of negative-corona discharge pulses in gases. 
The work on $\mathrm{S}_{2} \mathrm{~F}_{10}$ detection was carried out in collaboration with scientists at the Oak Ridge National Laboratory and results have already been reported in one archival [9] and two conference papers [10,11]. The work on stochastic behavior of corona pulses involved participation by guest scientists from the Plasma Research Institute of India and Old Dominion University. Results from the latter investigation have appeared in one archival [12] and two conference papers [13,14]. Three other archival papers covering different aspects of this work have also recently been submitted for publication [15-17].

\subsection{Detection of Trace $\mathrm{S}_{2} \mathrm{~F}_{10}$ in $\mathrm{SF}_{6}$}

The ability to detect gaseous disulfur decafluoride $\left(\mathrm{S}_{2} \mathrm{~F}_{10}\right)$ at trace levels in sulfur hexafluoride $\left(\mathrm{SF}_{6}\right)$ has become of interest because of its known high level of toxicity [18-22] and because of recent measurements [23,24] and calculations [25, 26] which show that $\mathrm{S}_{2} \mathrm{~F}_{10}$ can be produced during electrical-discharge activity in $\mathrm{SF}_{6}$. The concern about $S_{2} F_{10}$ production in $\mathrm{SF}_{6}$ stems in part from the increasingly wide spread use of $\mathrm{SF}_{6}$ as a gaseous dielectric in high-voltage electric-power transmission systems. The Occupational Safety and Health Administration (OSHA) has recently set peak exposure limits (PEL) for $\mathrm{S}_{2} \mathrm{~F}_{10}$ at $10 \mathrm{ppb}$ [27]. However, due to the difficulty of detecting low concentrations of $\mathrm{S}_{2} \mathrm{~F}_{10}$ in $\mathrm{SF}_{6}$, enforcement of the PEL has been stayed until the development of suitable detection techniques.

There are previous preliminary reports by Janssen $[28,29]$ of having achieved a detection sensitivity for $\mathrm{S}_{2} \mathrm{~F}_{10}$ in helium gas of $0.04 \mathrm{ppb}$ by using a gas chromatograph equipped with an electron capture detector. In order to achieve this level of sensitivity, however, it was necessary to employ a complex $\mathrm{S}_{2} \mathrm{~F}_{10}$ enrichment procedure requiring long analysis times ( $\sim 45$ minutes). When $\mathrm{SF}_{6}$ is a major constituent of the gas in which $S_{2} F_{10}$ is to be detected, the sensitivity for $S_{2} F_{10}$ detection drops dramatically (to $>10 \mathrm{ppb}$ ) despite the enrichment process. This drop in sensitivity is a consequence of increased background signal in the detector output due to the favorable electron attaching properties of the residual $S_{6}$ which interferes with the $S_{2} F_{10}$ signal. Reduced sensitivity for $\mathrm{S}_{2} \mathrm{~F}_{10}$ detection in the presence of $\mathrm{SF}_{6}$ also occurs when flame photometric detectors [28] or thermal conductivity detectors [11] are used.

Mass-spectrometric detection, when coupled to a gas chromatograph, suffers from the specific limitation that $S_{2} F_{10}$ has a positive-ion mass spectrum which is very similar to that of $\mathrm{SF}_{6}$, especially at the standard electron-impact energy of $70 \mathrm{eV}[29,30,31]$. For electron-impact energies greater than $20 \mathrm{eV}$, all of the significant ions that appear in the $S_{2} F_{10}$ mass spectrum also appear in the $S_{6}$ spectrum. Thus, even when chromatographic separation of $\mathrm{S}_{2} \mathrm{~F}_{10}$ from $\mathrm{SF}_{6}$ is used, ions from $\mathrm{SF}_{6}$ can sufficiently increase the background level to interfere with $S_{2} F_{10}$ detection. Recent claims [32] of using a mass spectrometer operated at low electron-impact energies to detect trace levels of $S_{2} F_{10}$ in $S F_{6}$ have been disputed [31]. 


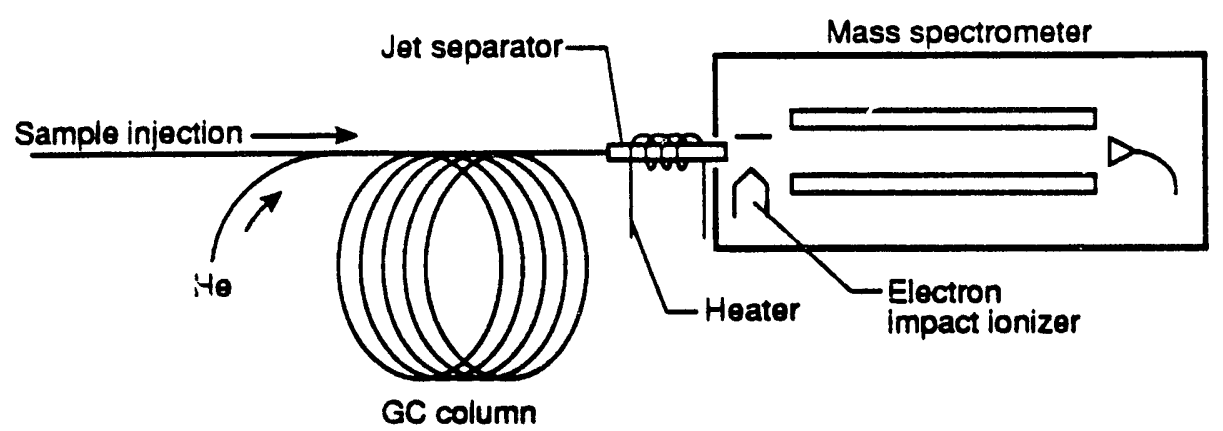

Figure 15. Schematic diagram of GC-MS system with a heated jet separator between the GC column and the ion source of the mass spectrometer. Heating of the interface between the GC and MS is essential for the sensitive detection of $S_{2} F_{10}$ in the presence of $S F_{6}$.

A new method is desiribed here in which a gas chromatograph-mass spectrometer (GC-MS) system is easily modified to allow detection of $S_{2} F_{10}$ in gaseous $S_{6}$ down to the ppb level without the necessity of a complex and time-consuming pre-enrichment procedure. With this method, a fraction of the $S_{2} F_{10}$ is converted to thionyl fluoride $\left(\mathrm{SOF}_{2}\right)$ in a heated jet-separator at the interface between the GC and MS. Because the major ions that appear in the $70-\mathrm{eV}$ mass spectrum of $\mathrm{SOF}_{2}$ differ from those in the $\mathrm{SF}_{6}$ spectrum, interference of $\mathrm{SF}_{6}$ with the $\mathrm{S}_{2} \mathrm{~F}_{10}$ peaks in single-ion chromatograms can be avoided. Elimination of the enrichment step significantly reduces the gas analysis time and improves the reliability of the detection method.

\subsection{1 ireasurement System}

The analytical method proposed for sensitive detection of $S_{2} F_{10}$ in $S F_{6}$ utilizes a GC-MS with a heated metal tube on the lower-pressure side of the interface between the GC and MS. As shown in figure 15, a jet separator serves as the heated metal tube in the GC-MS system used to obtain the results presented here. The jet separator is a standard design consisting of a stainless steel tube of $8 \mathrm{~cm}$ length and $5 \mathrm{~mm}$ diameter which is heated to a temperature of about $180^{\circ} \mathrm{C}$. As discussed later, a fraction of the $\mathrm{S}_{2} \mathrm{~F}_{10}$ undergoes thermal decomposition in the heated tube and converts to $\mathrm{SOF}_{2}$ via surface-catalyzed wall reactions with $\mathrm{H}_{2} \mathrm{O}$. The $\mathrm{SOF}_{2}$ can then be detected by the mass spectrometer without interference by the ion signals from $\mathrm{SF}_{6}$. The efficiency of the conversion is not known but appears, from experiments discussed below, to depend on the length, shape, and temperature of the heated surface upon which conversion takes place. The conversion efficiency is most sensitive to the tube temperature and relatively insensitive to details of the tube geometry and material composition. A high sensitivity for $\mathrm{S}_{2} F_{10}$ detection is obtainable with a wide range of heated inlet tube configurations. 
The GC-MS system used for the experiments presented here is a Hewlett Packard ${ }^{1}$ 5992A with a Hewlett Packard jet separation inlet. In this particular instrument the jet separator is heated by the diffusion pump heater. For other svstems the conversional region may be heated externally. The GC column is similar to that described by Hanrahan and Patterson [33], namely a $24^{\prime} \times 1 / 8^{\prime \prime}$ Teflon tube containing $30 \%$ SP-2100 (Supelco) on 80/100 chromosorb WAW. The column was operated using helium as a carrier gas $(20-30 \mathrm{ml} / \mathrm{min}$. How rate) which had not been predried. The $\mathrm{GC}$ oven temperature was maintained between 25 and $50^{\circ} \mathrm{C}$ and the sample injections were made using a gas-tight syringe. Detection sensitivities for $\mathrm{S}_{2} \mathrm{~F}_{10}$ in $\mathrm{SF} \mathrm{F}_{6}$ of nearly $1 \mathrm{ppb}$ were obtainable using the instrument described above.

Pure $S_{2} F_{10}$ samples, synthesized at Clemson University, were provided to us as liquid under its own vapor pressure ( $\sim 600$ torr at room temperature) in $30 \mathrm{ml}$ stainless steel cylinders. Identity and purity of the sample were established by IR spectroscopy and GC-MS analysis. Gas-phase reference mixtures were prepared immediately prior to use, either by injection or needle valve, in argon $(99.999 \%)$ or $\mathrm{SF}_{6}(99.99 \%)$ in a stainless steel vacuum manifold housed in a vented hood. The argon and $\mathrm{SF}_{6}$ were not dried before use.

\subsubsection{Data Acquisition and Analysis}

Data acquisition was performed using the manufacturer-supplied software and analysis was performed using software developed in-house. For $S_{2} F_{10}$ samples in buffer gases other than $\mathrm{SF}_{6}$, trace amounts of $\mathrm{S}_{2} \mathrm{~F}_{10}$ could be detected by single ion monitoring of $\mathrm{SF}_{5}^{+}, \mathrm{SF}_{4}^{+}, \mathrm{SF}_{3}^{+}, \mathrm{SF}_{2}^{+}$and $\mathrm{F}^{+}(\mathrm{m} / z=127,108,89,70$, and 19) produced by direct ionization of $S_{2} F_{10}[31]$. When mixed with $S_{6}$, sensitive $S_{2} F_{10}$ detection required the monitoring of ion signals characteristic of the $\mathrm{SOF}_{2}$ produced by surface conversion in the heated inlet tube mentioned above. These ions include $\mathrm{SOF}_{2}^{+}$, $\mathrm{SOF}^{+}$, or $\mathrm{SO}^{+}(m / z=86,67$, and 48$)$. For the data presented here, as many as 8 ions could be monitored at a given time with dwell times ranging from 10 to $200 \mathrm{~ms}$.

The response of the GC-MS to $S_{2} F_{10}$ is defined here to be the area under the peak identified with this species in a chromatogram. This area was computed from the GC-MS data by numerical integration after performing a background subtraction. No attempts were made to "smooth" or average data and unless specified, the data presented here corresponds to chromatograms from single sample injections. Retention time was measured from the time of sample injection.

\footnotetext{
${ }^{1}$ The identification of commercial materials and their sources is made to describe the experiment adequately. In no case does this identification imply recommendation by the National Institute of Standards and Technology, nor does it imply that the product is the best available.
} 


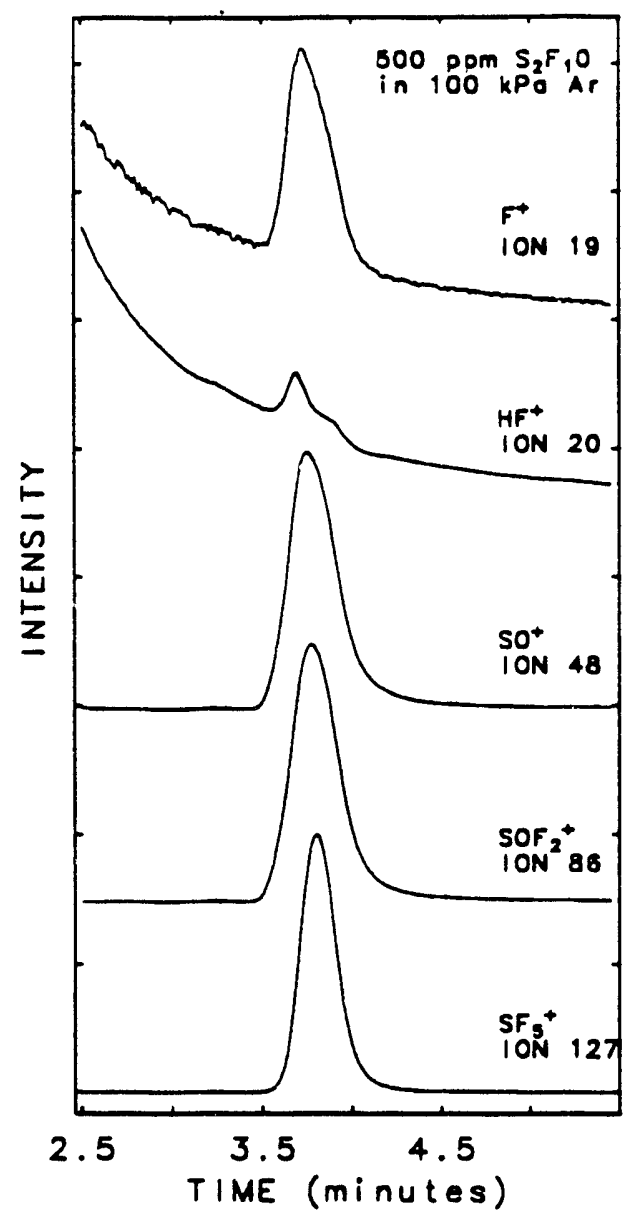

Figure 16. Chromatograms of representative ion signals due to ions from direct ionization of $S_{2} F_{10}$ and from ionization of surface conversion products for a $500 \mathrm{ppm}$ sample of $S_{2} F_{10}$ in $100 \mathrm{kPa}$ argon buffer gas.

\subsubsection{GC-MS Results and Calibration}

When a gas sample containing $\mathrm{S}_{2} \mathrm{~F}_{10}$ is injected into the column of the GC-MS in which the interface is heated, the conversion of $S_{2} F_{10}$ into SOF 2 discussed in the next section becomes evident from single-ion chromatograms at $m / z=48,67$, and 86 $\left(\mathrm{SO}^{+}, \mathrm{SOF}^{+}\right.$, and $\left.\mathrm{SOF}_{2}^{+}\right)$. Examples of such chromatograms are shown in figure 16 for a $500 \mathrm{ppm}$ sample of $\mathrm{S}_{2} \mathrm{~F}_{10}$ in argon. Ions produced by direct ionization of $\mathrm{S}_{2} \mathrm{~F}_{10}$ are observed $\left(\mathrm{F}^{+}\right.$and $\mathrm{SF}_{5}^{+}$) as are ions from $\mathrm{SOF}_{2}$ produced by surface conversion $\left(\mathrm{SOF}_{2}^{+}\right.$and $\left.\mathrm{SO}^{+}\right)$.

The $m / z=20$ chromatogram in figure 16 also exhibits a peak at the retention time characteristic for $S_{2} F_{10}$, thus indicating that $H F$ is also a by-product of $S_{2} F_{10}$ decomposition in the heated tube. This peak, however, exhibits structure which can be accounted for by interference from HF originally present as a contaminant in the sample mixture. Analysis of dilute HF mixtures showed that HF has nearly the same 


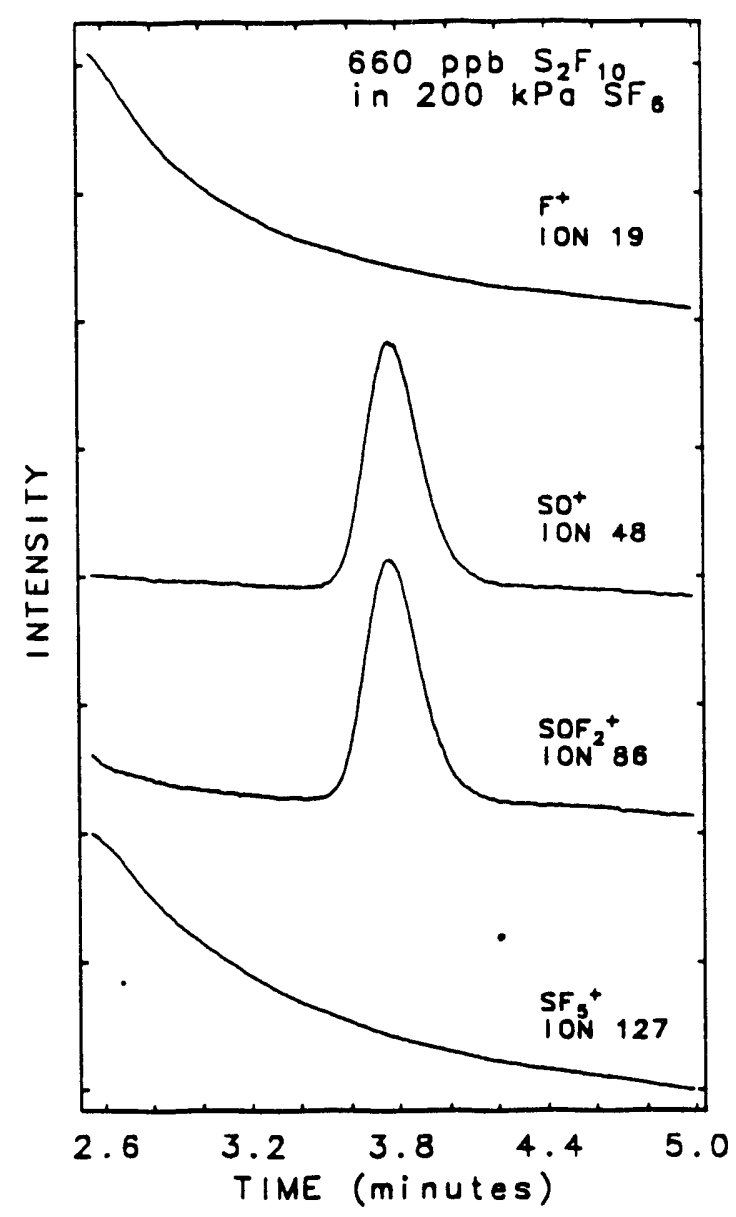

Figure 17. Single ion chromatograms of a $660 \mathrm{ppb} \mathrm{S}_{2} F_{10}$ sample in $200 \mathrm{kPa}$ argon buffer gas.

retention time as $\mathrm{S}_{2} \mathrm{~F}_{10}$ for the column conditions used here. Thus, although $\mathrm{HF}$ may be formed by thermal decomposition of $\mathrm{S}_{2} F_{10}$ in the heated gas inlet tube, it cannot, by itself, be relied upon to give an unambiguous indication of $S_{2} F_{10}$.

For the results shown in figure 16 , ions due to direct ionization of $\mathrm{S}_{2} \mathrm{~F}_{10}$ are observable because no $\mathrm{SF}_{6}$ was present in the sample. However, in the presence of $\mathrm{SF}_{6}, \mathrm{~S}_{2} \mathrm{~F}_{10}$ signals in the single-ion chromatograms at $\mathrm{m} / z=19$ and 127 are obscured by the $\mathrm{SF}_{6}$ contribution to the signal at these masses. This is demonstrated by data presented in figure 17 from a gas sample containing $660 \mathrm{ppb} \mathrm{S}_{2} \mathrm{~F}_{10}$ in $\mathrm{SF}_{6}$. These results show the effect of $\mathrm{SF}_{6}$ in limiting the sensitivity for $\mathrm{S}_{2} \mathrm{~F}_{10}$ detection when conventional mass-spectrometric monitoring is used, and clearly show the advantages of using the $\mathrm{S}_{2} \mathrm{~F}_{10}$ conversion technique discussed here.

Figure 18 shows a chromatogram for $\mathrm{m} / \mathrm{z}=86$ for an "unknown" $\mathrm{SF} 6$ sample from a container in which the gas had been subjected to a weak electrical corona discharge. The discharge produced trace amounts of the oxyfluorides $\mathrm{SOF}_{2}, \mathrm{SOF}_{4}$, and $\mathrm{SO}_{2} \mathrm{~F}_{2}$ in 


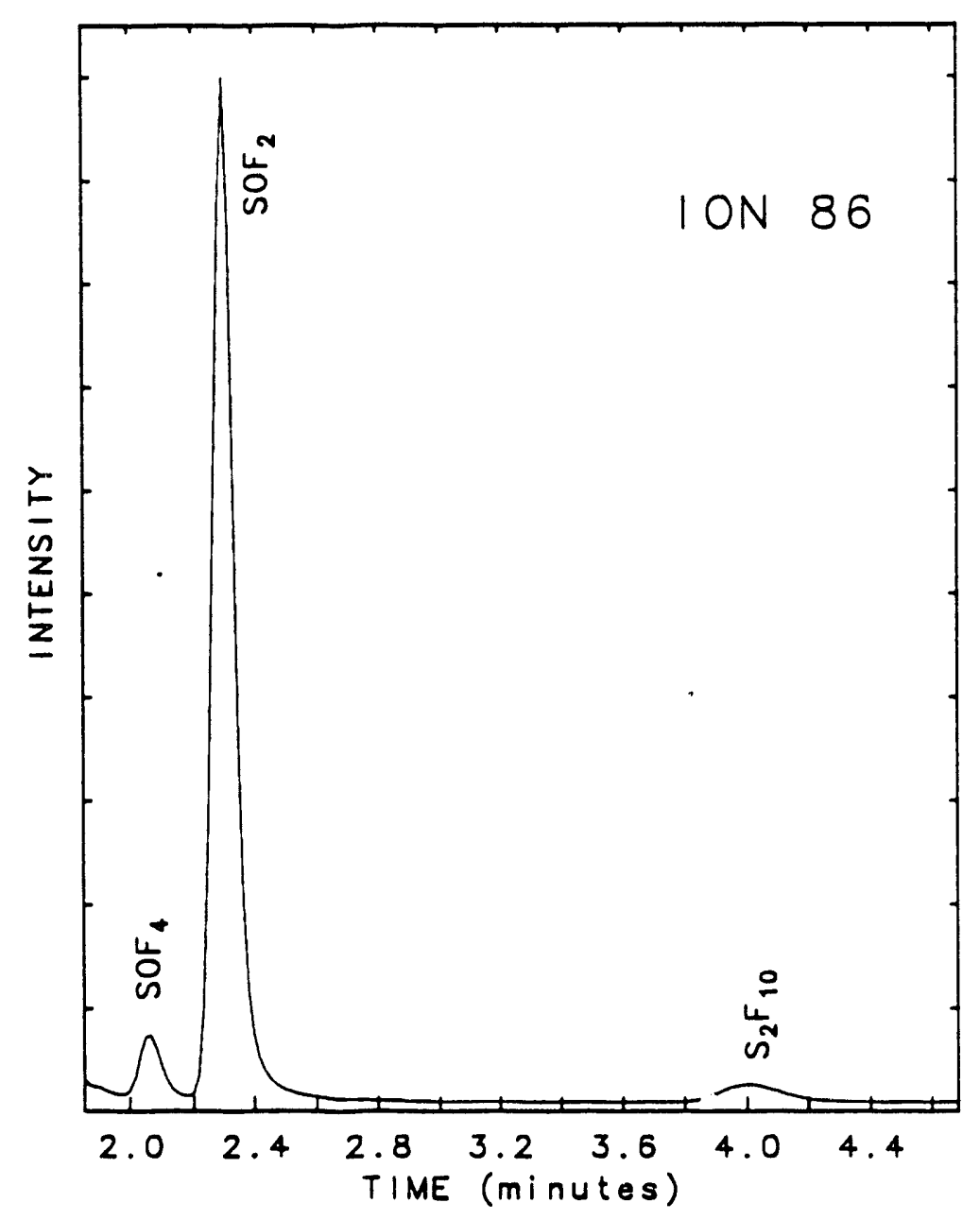

Figure 18. Single ion chromatogram of an "unknown" sample of $S_{6}$ that was partially decomposed in a dc corona discharge. 


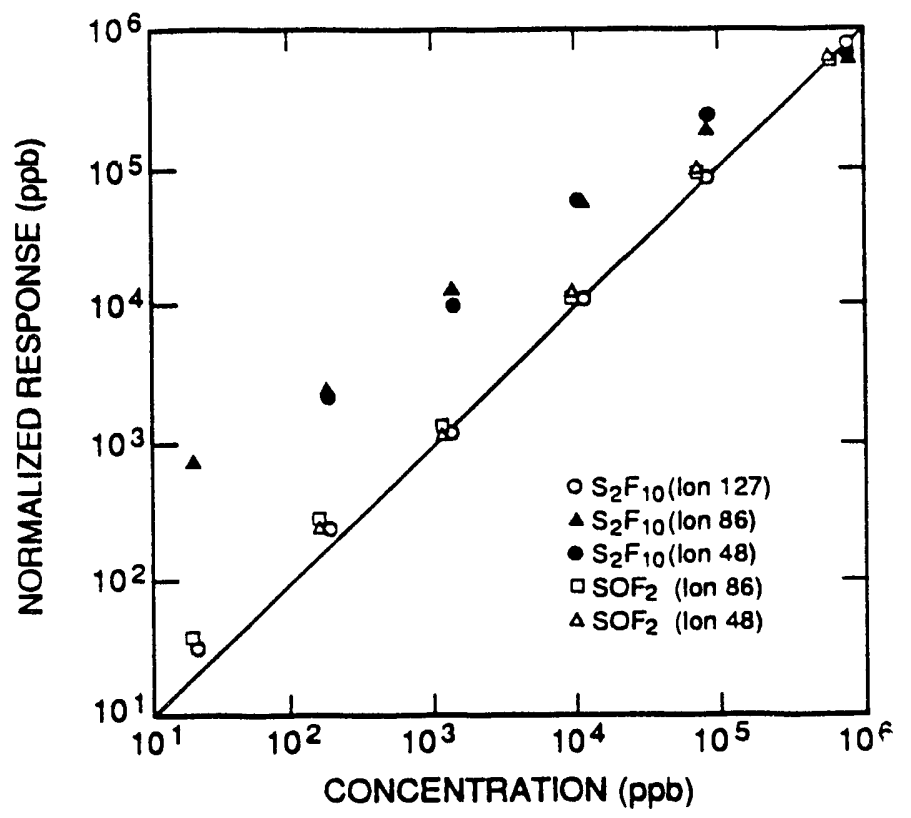

Figure 19. Detection response curves for various concentrations of $\mathrm{S}_{2} \mathrm{~F}_{10}$ and $\mathrm{SOF}_{2}$ in argon using a chromosorb GC column discussed in the text. Open symbols represent detection of $\mathrm{S}_{2} \mathrm{~F}_{10}$ and $\mathrm{SOF}_{2}$ by direct ionization, while closed symbols represent detection of $S_{2} F_{10}$ by ionization of $S O F_{2}$ produced by surface catalysis. The measured responses were normalized to the observed responses of a reference sample containing $750 \mathrm{ppm} \mathrm{S}_{2} \mathrm{~F}_{10}$ and $750 \mathrm{ppm} \mathrm{SOF}_{2}$ in argon. Similar response curves are obtained for samples diluted in $\mathrm{SF}_{6}$.

addition to $\mathrm{S}_{2} \mathrm{~F}_{10}$. It is seen that the retention times of the $\mathrm{SOF}_{2}$ and $\mathrm{SOF}_{4}$ produced by the discharge are much shorter than for $S_{2} F_{10}$ (2.1 and 2.3 minutes compared to 4.0 minutes). The oxyfluorides are known from previous work [34-36] to be the major gaseous by-products from decomposition of $\mathrm{SF}_{6}$ in corona discharges when at least trace levels of oxygen and water vapor are present. The production of $\mathrm{S}_{2} \mathrm{~F}_{10}$ in $\mathrm{SF}_{6}$ electrical discharges has only recently been verified $[23,24]$.

Under the conditions used to obtain the data in figures 16 and 17 , the response of the GC-MS was measured as a function of the $S_{2} F_{10}$ concentration in reference gas samples. Typical response curves obtained using known concentrations of $\mathrm{S}_{2} \mathrm{~F}_{10}$ mixed with argon are shown in figure 19 for the indicated $m / z$ values. The $S_{2} F_{10}$ response is compared with that for $\mathrm{SOF}_{2}$ which was also added to the mixture in known concentrations. The $S_{2} F_{10}$ response curves for $m / z=48$ and 86 , corresponding to the chemical conversion of $\mathrm{S}_{2} \mathrm{~F}_{10}$ into $\mathrm{SOF}_{2}$, are seen to be distinctly nonlinear. The deviation from linearity is greatest at the highest $\mathrm{S}_{2} \mathrm{~F}_{10}$ concentrations and indicates a saturation effect possibly associated with depletion of $\mathrm{H}_{2} \mathrm{O}$ from the heated jet separator tube surface during the conversion of $\mathrm{S}_{2} \mathrm{~F}_{10}$ into $\mathrm{SOF}_{2}$. By contrast, the $\mathrm{S}_{2} \mathrm{~F}_{10}$ response curve for $m / z=127$, corresponding to unreacted $\mathrm{S}_{2} \mathrm{~F}_{10}$, is seen to be nearly linear over 5 orders of magnitude. Likewise the responses to $\mathrm{SOF}_{2}$ originally present in the sample at $m / z=48$ and 86 are also nearly linear. The small deviations 
from linearity observed at low concentrations $(<200 \mathrm{ppb})$ may be attributable to uncertainties in the preparation of samples by successive dilutions.

The shape and slope of the response curves for the $S_{2} F_{10}$ conversion process are quite reproducible for a given set of instrument operating conditions. Nevertheless, when attempting accurate measurements of $S_{2} F_{10}$ concentrations in $S F_{6}$, frequent checks on the GC-MS calibration may be necessary and reference sample concentrations should ideally be as close to the unknown sample concentration as possible. The response of the present GC-MS system to $S_{2} F_{10}$ was periodically checked and found to be stable for long periods of time (typically more than a day). When performing a quantitative analysis of $\mathrm{S}_{2} \mathrm{~F}_{10}$ content in $\mathrm{SF}_{6}$, it is desirable to bracket the unknown sample injection with reference injections of comparable concentration.

It should be noted that although the response at high $S_{2} F_{10}$ concentrations shows evidence of $\mathrm{H}_{2} \mathrm{O}$ removal, the surface apparently recovers to its former condition in a relatively short time $(\sim 5-10 \mathrm{~min}$.) as indicated by responses from successive injections of samples at high concentrations $(\sim 100 \mathrm{ppm})$. In general, the larger the quantity of $\mathrm{S}_{2} \mathrm{~F}_{10}$ injected, the longer the surface recovery time of the inlet tube. For samples containing less than $10 \mathrm{ppm} \mathrm{S}_{2} \mathrm{~F}_{10}$, the recovery time of the system used here was found to be less than the typical $S_{2} F_{10}$ retention time of $4 \mathrm{~min}$.

The limit of $\mathrm{S}_{2} \mathrm{~F}_{10}$ detection in $\mathrm{SF}_{6}$ depends, of course, on the operating conditions of the GC-MS equipped with the heated inlet line. For the instrument used here, under conditions identical to those used to obtain the results shown in figures 16,17 and 18, the limit of detection (signal-to-noise ratio $=2$ ) was estimated to be about $2 \mathrm{ppb}$ for a $2 \mathrm{ml}$ injection at $200 \mathrm{kPa}$. Figure 20 shows an example of a result obtained for a $4 \mathrm{ppb}$ mixture of $\mathrm{S}_{2} \mathrm{~F}_{10}$ in $\mathrm{SF}_{6}$ which gives an indication of the limit of detection sensitivity for this instrument. The single-ion chromatogram at $m / z=86$ is an average of data from four injections each with a volume of $2 \mathrm{ml}$ at a total gas pressure of $200 \mathrm{kPa}$. The $\mathrm{S}_{2} \mathrm{~F}_{10}$ peak is statistically significant and is indicated by the vertical arrow. The presence of $\mathrm{S}_{2} F_{10}$ in $\mathrm{SF}_{6}$ at the $10 \mathrm{ppb}$ level (the PEL) gives a much larger peak than that shown in figure 20 and is easily detected using a single injection. If necessary, additional improvements in signal-to-noise ratios needed for higher sensitivity applications can be achieved through more signal processing, (i.e., averaging of results from more injections coupled with appropriate background subtraction) or using heated MS inlet-tube conditions that optimize the efficiency of $\mathrm{S}_{2} F_{10}$ conversion into $\mathrm{SOF}_{2}$.

\subsubsection{Mass-Spectrometric Investigation of $S_{2} F_{10}$ Pyrolysis}

In order to determine the optimum operating temperature range for the heated gas inlet tube and to better understand the thermal $S_{2} F_{10}$ conversion mechanism discussed in the previous section, it was necessary to investigate the pyrolysis of $\mathrm{S}_{2} \mathrm{~F}_{10}$ on heated metal surfaces at low pressure and at different temperatures. The 


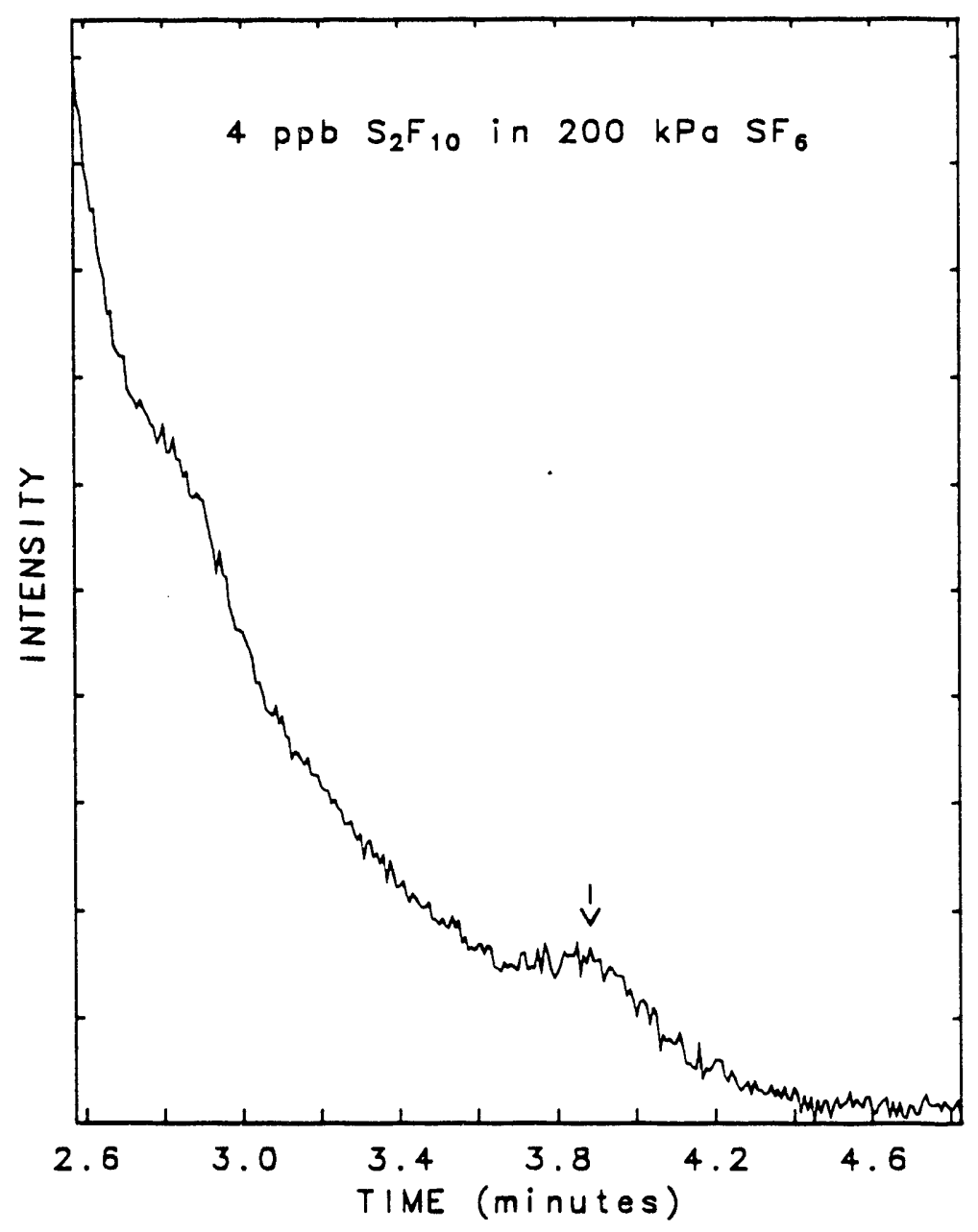

Figure 20. Single ion chromatogram (ion 86 ) of $4 \mathrm{ppb} \mathrm{S}_{2} F_{10}$ in $200 \mathrm{kPa} \mathrm{SF} 6$. 


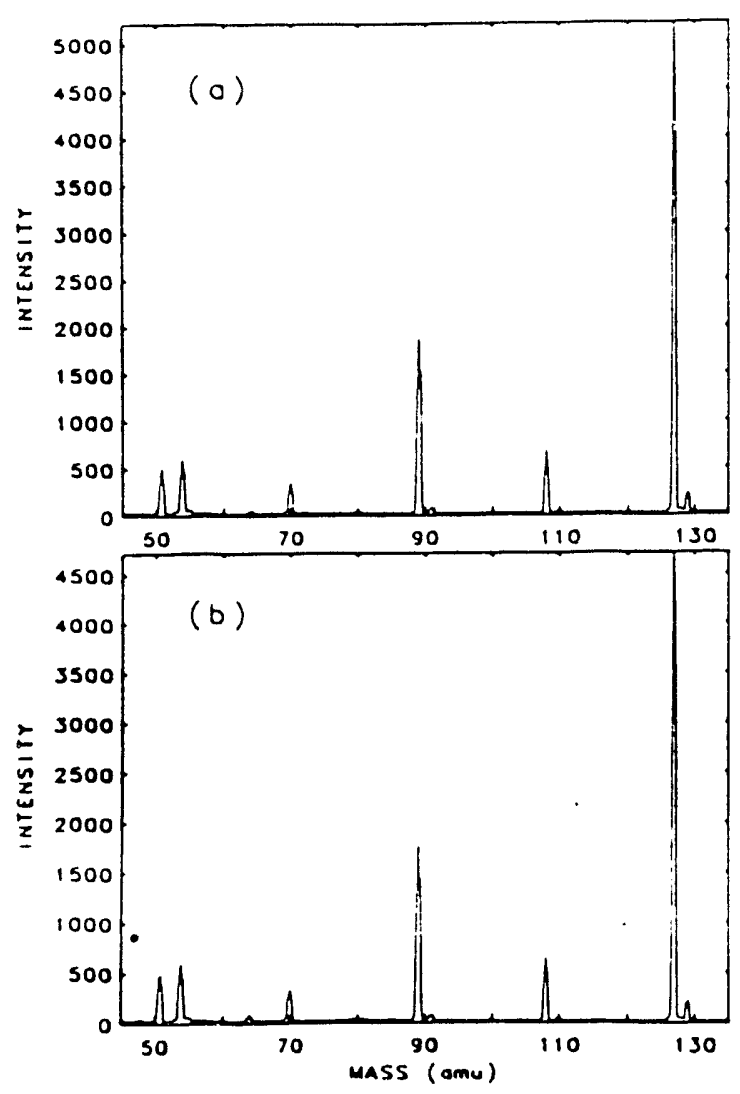

Figure 21. Electron-impact mass spectra of $1500 \mathrm{ppm} \mathrm{SF}_{6}$ in argon buffer gas at (a) $30^{\circ} \mathrm{C}$ and (b) $220^{\circ} \mathrm{C}$.

specific purposes of this investigation were to: 1) show evidence that it is surface chemistry that is involved in the $\mathrm{S}_{2} \mathrm{~F}_{10}$ conversion; 2) indicate the temperature range over which this conversion is effective; 3 ) demonstrate that $\mathrm{SF}_{6}$ does not chemically decompose in this temperature range; and 4) provide an independent verification that $\mathrm{SOF}_{2}$ is indeed one of the products of the $\mathrm{S}_{2} \mathrm{~F}_{10}$ conversion process.

An Extrel quadrupole mass spectrometer was used for these measurements. and the sample gas was admitted to the MS via a heated stainless steel bellows connected to a variable leak valve. The temperature of the bellows could be varied from 20 to $300^{\circ} \mathrm{C}$ and the mass spectra could be observed as a function of the temperature of the inlet system. The gas pressure in the bellows was estimated to be lower than 1 millitorr $(\sim 0.13 \mathrm{~Pa})$ and the pressure in the MS ion-source was below $5 \times 10^{-5}$ torr.

Shown respectively in figures 21 and 22 are mass spectra obtained from mixtures of

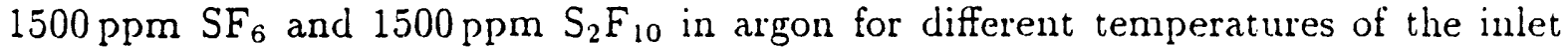
bellows. At $30^{\circ} \mathrm{C}$, the mass spectra of $S_{2} F_{10}$ and $S F_{6}$ are seen to be nearly identical in agreement with previously published data $[30,31,37,38]$. Other than minor differences in the rclative intensities of the $S_{x}^{+}$ions $(x=1-4)$, the only significant difference between the mass spectra of $S_{2} F_{10}$ and $S F_{6}$ is the appearance of a peak at 

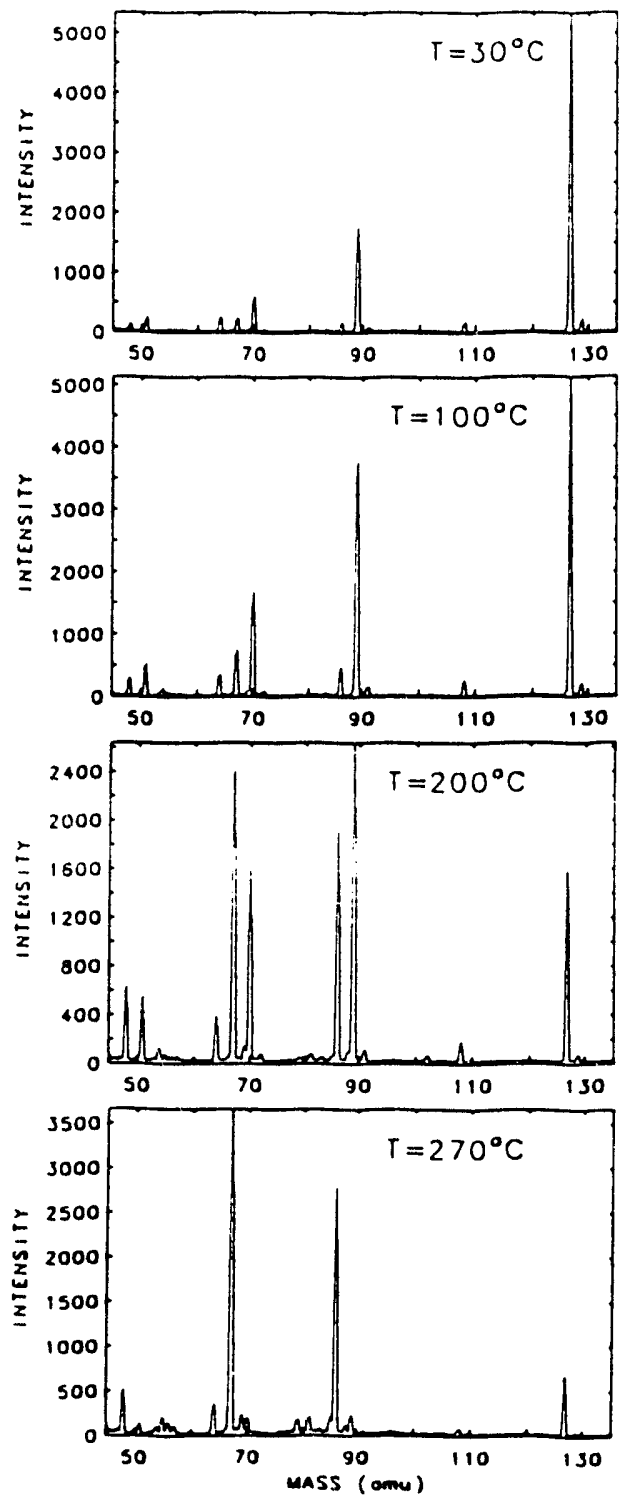

Figure 22. Electron-impact mass spectra of $1500 \mathrm{ppm} \mathrm{S}_{2} \mathrm{~F}_{10}$ in argon buffer gas at various temperatures. 
$m / z=54\left(\mathrm{SF}_{4}^{++}\right)$in the $\mathrm{SF}_{6}$ spectrum which is not present in $\mathrm{S}_{2} \mathrm{~F}_{10}$ spectrum. Peaks at masses $48,64,67$, and 86 in the $S_{2} F_{10}$ spectrum at $30^{\circ} \mathrm{C}$ are due to small impurities of $\mathrm{SO}_{2}$ and $\mathrm{SOF}_{2}$ present in the $\mathrm{S}_{2} \mathrm{~F}_{10}$ sample. At $220^{\circ} \mathrm{C}$, the $\mathrm{SF}_{6}$ mass spectrum remains essentially unchanged, indicating that $\mathrm{SF}_{6}$ does not undergo a measurable chemical transformation in the heated inlet tube. The $S_{2} F_{10}$ spectrum, on the other hand, exhibits a significant change for temperatures above about $100^{\circ} \mathrm{C}$. In going from $30^{\circ} \mathrm{C}$ to $200^{\circ} \mathrm{C}$, the ratio of the $\mathrm{SF}_{3}^{+}(\mathrm{m} / z=89)$ to $\mathrm{SF}_{5}^{+}(\mathrm{m} / z=127)$ peaks in the $\mathrm{S}_{2} \mathrm{~F}_{10}$ mass spectrum increases indicating conversion of $\mathrm{S}_{2} \mathrm{~F}_{10}$ into $\mathrm{SF}_{4}$ or $\mathrm{SF}_{5}$. Peaks associated with $\mathrm{SOF}_{2}$ at $m / z=48,67$, and 86 become more prominent above $100^{\circ} \mathrm{C}$ and dominate the spectrum at $270^{\circ} \mathrm{C}$, indicating that $\mathrm{S}_{2} \mathrm{~F}_{10}$ is nearly completely destroyed at this temperature with $\mathrm{SOF}_{2}$ as the predominant stable by-product.

Tests performed using different inlet tubes appear to indicate that, at any given temperature, the conversion of $\mathrm{S}_{2} \mathrm{~F}_{10}$ into $\mathrm{SOF}_{2}$ becomes more efficient as the effective surface area encountered by the incoming gas is increased, e.g., a bellows gives a more complete conversion than a straight tube of the same length. This trend is consistent with chemical conversion via a surface catalyzed reaction. There are indications that some surface materials may be more effective than others in converting $S_{2} F_{10}$ to $\mathrm{SOF}_{2}$, however, an extensive investigation appeared to be unwarranted in view of the relatively high efficiency achieved with commonly used stainless steel. Temperature and presence of adsorbed water appear to be more important than either surface configuration or composition.

The detailed mechanisms of $\mathrm{S}_{2} \mathrm{~F}_{10}$ conversion to $\mathrm{SOF}_{2}$ on heated surfaces are not presently known. It is speculated that the process involves thermally induced dissociation of $\mathrm{S}_{2} \mathrm{~F}_{10}$ along the weak sulfur-sulfur bond followed by a fast reaction of $\mathrm{SF}_{5}$ with $\mathrm{H}_{2} \mathrm{O}$ adsorbed on the surface, i.e.,

$$
\begin{gathered}
\mathrm{S}_{2} \mathrm{~F}_{10} \rightarrow \mathrm{SF}_{5}+\mathrm{SF}_{5} \\
\mathrm{SF}_{5}+\mathrm{H}_{2} \mathrm{O} \rightarrow \mathrm{SOF}_{2}+2 \mathrm{HF}+\mathrm{F} .
\end{gathered}
$$

Previous work [39-41] has shown that $\mathrm{S}_{2} \mathrm{~F}_{10}$ is thermally unstable in the gas phase at temperatures above $100^{\circ} \mathrm{C}$. The thermal unimolecular gas-phase decomposition rate at high pressures [39] has a temperature $(T)$ dependence given by:

$$
k=3 \times 10^{19} \exp (-46400 / R T),
$$

which is based on reaction data [39] obtained in the temperature range 435-454.5 K ( $R T$ is given in units of calories and $k$ in $\mathrm{s}^{-1}$ ). Assuming this expression applies at room temperature, the half-life of $S_{2} F_{10}$ in the gas phase is predicted to be about $10^{7}$ years. The half-life drops dramatically to $10^{2}$ seconds at $475 \mathrm{~K}$. However, the $\mathrm{S}_{2} \mathrm{~F}_{10}$ decomposition rate implied by the present mass-spectrometric results is significantly higher than predicted by the above gas-phase rate and unlikely to apply at the low pressures typically encountered here in the heated inlet tube, further supporting the likelihood of a surface catalyzed reaction mechanism. 


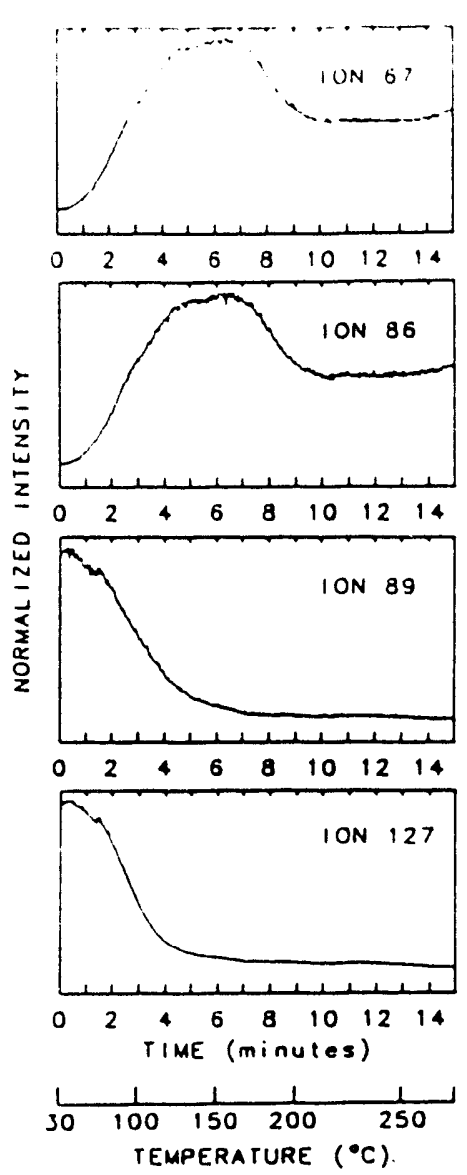

Figure 23. Signal intensity of several selected ions from a $1500 \mathrm{ppm} \mathrm{S}_{2} \mathrm{~F}_{10}$ sample in argon as a function of time as the inlet temperature is rapidly increased from room temperature to $270^{\circ} \mathrm{C}$.

An indirect indication of the role of adsorbed water was obtained from measurements made with the quadrupole mass spectrometer under conditions where the inlet line temperature was allowed to rise relatively rapidly after the $A r / S_{2} F_{10}$ mixture was introduced. The mass spectra obtained in this way differ from those shown in figure 22 which correspond to equilibrated conditions achieved after keeping the inlet line at the indicated temperatures for many minutes. The results obtained from monitoring selected ions as a function of time under the "transient" conditions of continuous rising inlet-line temperature are shown in figure 23. Presented in this figure are timedependent peak intensities for ions at $\mathrm{m} / z=S 6$ and 67 which are indicators of $\mathrm{SOF}_{2}$; $m / z=127$, indicator of $S_{2} F_{10}$ and $S_{6}$; and $m / z=89$, indicator of $S_{2} F_{10}, S_{6}$, and $\mathrm{SF}_{4}$. Unlike the results given in figure 22 , the yield of $\mathrm{SOF}_{2}$ is seen to go through a maximum at a time of 6 minutes, which corresponds to a temperature of $160^{\circ} \mathrm{C}$. The data for $m / z=127$ indicate that $S_{2} F_{10}$ is nearly completely decomposed at this temperature, i.e., the intensity for this ion ceases to decrease significantly beyond this temperature. The $m / z=12 T$ signal that appears at higher temperatures is 
undoubtedly associated with $\mathrm{SF}_{6}$ which can be a product of $\mathrm{S}_{2} \mathrm{~F}_{10}$ decomposition. As noted by Herron [41] (see also Ref. [42]), $\mathrm{SF}_{4}$ and $\mathrm{SF}_{6}$ can be formed in the gas phase by the disproportionation reaction

$$
\mathrm{SF}_{5}+\mathrm{SF}_{5} \rightarrow \mathrm{SF}_{4}+\mathrm{SF}_{6}
$$

As the temperature increases, the $m / z=89$ signal does not fall off quite as rapidly as the $m / z=127$ signal, thus suggesting formation of $\mathrm{SF}_{4}$ whose primary electron impact ion is $\mathrm{SF}_{3}^{+}$. The increase in $\mathrm{SOF}_{2}$ production with time can be attributed to an increase in the decomposition rate of $\mathrm{S}_{2} \mathrm{~F}_{10}$ and an increase in the surface hydrolysis rate of the decomposition products as the surface temperature is increased. However, as the amount of water adsorbed on the surface decreases due to heating, the rate of $\mathrm{S}_{2} \mathrm{~F}_{10}$ conversion into $\mathrm{SOF}_{2}$ drops. It should be noted that at the highest temperatures the production of $\mathrm{SOF}_{2}$ has decreased with no corresponding increase in any of the other compounds which are being monitored. This suggests that at high temperatures the $S_{2} F_{10}$ or its by-products are being converted into compounds which are presently unidentified.

Although there is evidence [43] that $\mathrm{SOF}_{2}$ hydrolyzes slowly in the gas phase at $300 \mathrm{~K}$ and may react with $\mathrm{H}_{2} \mathrm{O}$ on surfaces, little is known about its decomposition at higher temperatures. These results indicate that there is an optimum temperature range at which conversion of $S_{2} F_{10}$ into $\mathrm{SOF}_{2}$ is most efficient. If the temperature is too low, the conversion rate is low, and if the temperature is too high, $\mathrm{SOF}_{2}$ itself may be destroyed.

\subsubsection{Gas-phase Impurities}

It should be realized that if gaseous species are present in the unknown sample that could react with $S_{2} F_{10}$ or its decomposition products at temperatures above $150^{\circ} \mathrm{C}$, uncertainties can be introduced in the quantitative analysis for $\mathrm{S}_{2} \mathrm{~F}_{10}$ by the method proposed here if the reference sample does not also contain these species in nearly the same amounts. It is known [44-47] that $S_{2} F_{10}$ can react at high temperatures with numerous gases such as $\mathrm{NO}, \mathrm{NO}_{2}, \mathrm{NO}_{2} \mathrm{Cl},\left(\mathrm{CF}_{3}\right)_{2} \mathrm{NONO}, \mathrm{CH}_{3} \mathrm{ONO}, \mathrm{Fe}(\mathrm{CO})_{5}$, ICl, $\mathrm{C}_{6} \mathrm{H}_{6}, \mathrm{C}_{2} \mathrm{H}_{4},\left(\mathrm{CH}_{3}\right)_{2} \mathrm{NH}, \mathrm{BCl}_{3}$, and $\mathrm{NH}_{3}$. If it is suspected that gases may be present in the unknown sample that can react with $\mathrm{S}_{2} \mathrm{~F}_{10}$ at temperatures above $150^{\circ} \mathrm{C}$, then care must be taken to assess the influence of these gases on the GC-MS response to $S_{2} F_{10}$. Ideally the reference sample should have the same, or nearly the same composition as the unknown sample. It was found here that the response of the GC-MS to $\mathrm{S}_{2} \mathrm{~F}_{10}$ was not significantly influenced by the presence of the oxyfluorides $\mathrm{SOF}_{2}, \mathrm{SO}_{2} \mathrm{~F}_{2}$, and $\mathrm{SOF}_{4}$ which are commonly formed together with $\mathrm{S}_{2} \mathrm{~F}_{10}$ during decomposition or oxidation of $\mathrm{SF}_{6}$.

Of particular importance is the presence of water vapor as a contaminant in a sample. The gas-phase water vapor present in the sample can affect the amount of adsorbed 
water on the inlet tube surface at any given time and thus the $S_{2} F_{10}$ to $S_{0} F_{2}$ conversion efficiency. Low water-vapor concentrations in gas samples did not show significant effects. However, it was observed that the addition of excessive amounts of water vapor to an $\mathrm{S}_{2} \mathrm{~F}_{10}$ sample can noticeably decrease the amount of $\mathrm{SOF}_{2}$ detected from $S_{2} F_{10}$ conversion at surface temperatures above about $120^{\circ} \mathrm{C}$. This is not understood but may be due to mechanisms which either inhibit the conversion if gas-phase water is present, or increase the rate of $\mathrm{SOF}_{2}$ decomposition (for example by hydrolysis [35]) at elevated temperatures. The reactivities of gas-phase $\mathrm{S}_{2} \mathrm{~F}_{10}$ and $\mathrm{SOF}_{2}$ toward water vapor at temperatures above $100^{\circ} \mathrm{C}$ are presently unknown and require more investigation.

\subsubsection{Reference Sample Stability}

Surface conversion of $\mathrm{S}_{2} F_{10}$ into $\mathrm{SOF}_{2}$ also occurs at room temperature, although at a much slower rate than observed at elevated temperatures. This decomposition raises questions about the long-term reliability of reference gas samples containing known amounts of $S_{2} F_{10}$ that are needed for calibration of the GC-MS. In order to assess the stability of $S_{2} F_{10}$ in reference samples, a series of tests were performed to monitor the content of $\mathrm{S}_{2} \mathrm{~F}_{10}$-containing cylinders over relatively long periods of time. Preliminary reports of these tests have already been given $[10,24]$.

It was found that the rate of $S_{2} F_{10}$ decomposition depended on such factors as temperature, cylinder material and surface-to-volume ratio, and moisture content. Data indicating the temperature and moisture content dependencies of $\mathrm{S}_{2} \mathrm{~F}_{10}$ decomposition in $150 \mathrm{ml}$ stainless-steel cylinders are shown respectively in figures 24 and 25 . Shown in these figures are measured $S_{2} F_{10}$ concentrations in argon as a function of time after mixture preparation. The measurements were performed using the GC-MS method described above, and the "aged" samples were compared with reference samples prepared immediately prior to the measurements. The "wet" sample $\left(\mathrm{H}_{2} \mathrm{O}\right.$ added $)$ results in figure 25 were obtained from a sample prepared by injecting $1 \mu \mathrm{l}$ of liquid water into the cylinder under vacuum prior to introducing the $\mathrm{Ar} / \mathrm{S}_{2} \mathrm{~F}_{10}$ gas mixture. The data presented in figures 24 and 25 clearly demonstrate that the $\mathrm{S}_{2} \mathrm{~F}_{10}$ decomposition rate increases with increasing temperature and moisture content.

The products of the $\mathrm{S}_{2} \mathrm{~F}_{10}$ decomposition were found to include $S O F_{2}$ and $\mathrm{SF}_{6}$, and thus it is speculated that the decomposition process involves a surface catalyzed reaction with adsorbed $\mathrm{H}_{2} \mathrm{O}$ that may be similar to that observed at higher temperatures in the heated $\mathrm{MS}$ gas inlet tube. The $\mathrm{SOF}_{2}$ produced from $\mathrm{S}_{2} \mathrm{~F}_{10}$ also appears to react slowly on the stainless-steel surface and convert to other products that have yet to be identified. Once $\mathrm{S}_{2} \mathrm{~F}_{10}$ disappears from the gas, $\mathrm{SOF}_{2}$ will also eventually disappear [38]. From analyses of the decomposed gas using MS and GCMS, it has not been possible to account for all of the sulfur originally present in the 


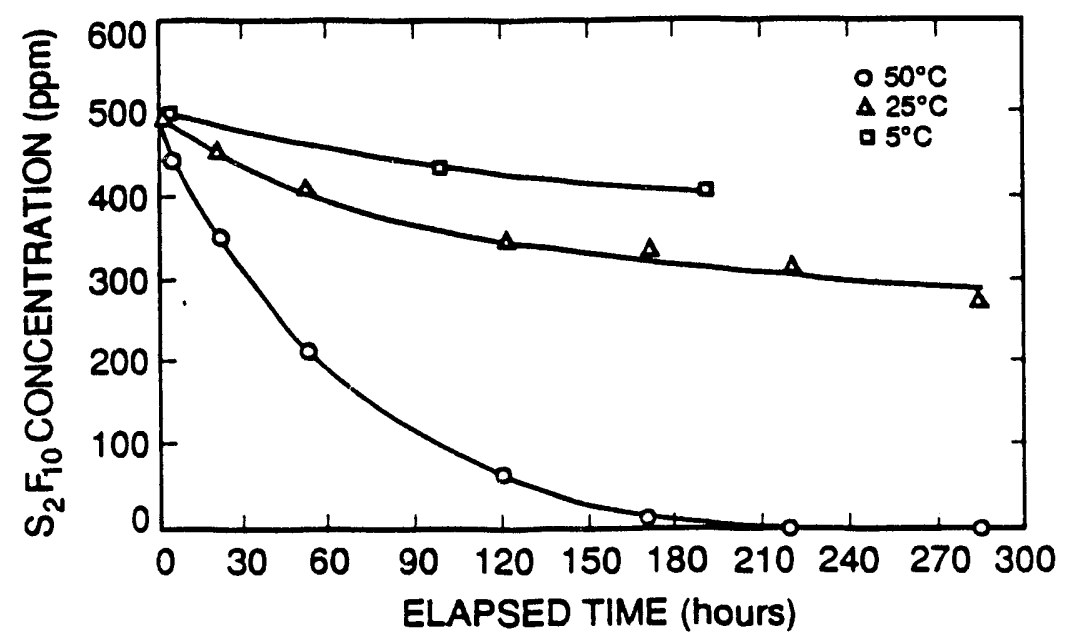

Figure 24. Low temperature dependence for decomposition of $500 \mathrm{ppm} \mathrm{S}_{2} F_{10}$ samples in . argon stored in $150 \mathrm{ml}$ stainless steel cylinders.

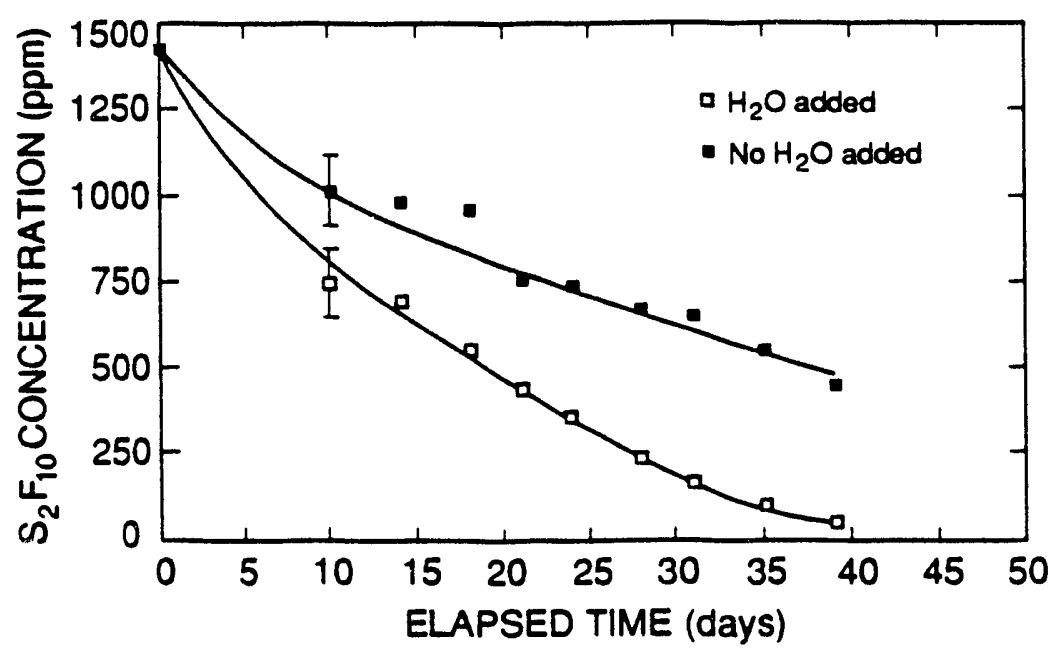

Figure 25. Decomposition of $S_{2} F_{10}$ as a function of time for two $1400 \mathrm{ppm}, \mathrm{S}_{2} \mathrm{~F}_{10}$ samples in $1 \mathrm{~atm} \mathrm{Ar}$ in $150 \mathrm{ml}$ stainless steel cylinders. For one sample ( $\square), 1 \mu \mathrm{l}$ of liquid water was injected into the cylinder prior to filling with gas. 
$\mathrm{S}_{2} \mathrm{~F}_{10}$. The only identified gaseous compound resulting from $\mathrm{S}_{2} \mathrm{~F}_{10}$ decomposition that contains sulfur and exhibits long-term stability is $\mathrm{SF}_{6}$. Generally, less than $15 \%$ of the sulfur can be accounted for by this product.

On the basis of the tests that were conducted, long-term storage of $S_{2} F_{10}$ reference samples in the gas phase cannot be recommended. It was found that samples stored under even the most favorable conditions exhibited significant decomposition over sufficiently long periods of time. It is recommended that reference samples be prepared immediately prior to gas analysis, under conditions that are as dry as possible, and in cylinders that are as large as possible.

Preliminary tests of commercial grade $S F_{6}\left(99.8 \%\right.$ purity) have indicated that $S_{2} F_{10}$ does not readily decompose in $\mathrm{SF}_{6}$-filled cylinders when compressed into the liquid phase. Analysis showed that $S_{2} F_{10}$ was present as an impurity at a level of about $40 \mathrm{ppb}$ in a 12-year old sample. It should be noted that $S_{2} F_{10}$ is a possible by-product from the commercial production of $\mathrm{SF}_{6}$ and can be present as a trace liquid impurity in the liquified $\mathrm{SF}_{6}$ which exists in pressurized cylinders. The results of analyzing $\mathrm{SF}_{6}$ stored as a liquid suggest that significant improvements might be achieved in maintaining sample stability by compressing reference samples into the liquid phase.

\subsection{Stochastic Properties of Pulsating Partial Discharges}

\subsubsection{Basic Considerations}

Partial-discharge (P-D) measurements have long been recognized as an important part of quality control for high-voltage apparatus. There is also increasing interest in the use of P-D measurement as a diagnostic tool for assessing insulation performance and integrity when subjected to electrical and mechanical stress. The measurement of P-D's has been applied to a wide range of electrical apparatus including rotating machines, power transformers, and both low- and high-voltage cables [48].

The P-D phenomenon under consideration here is a self-quenching discharge that is localized in regions of high electrical stress within insulating systems. The high-stress regions might, for example, be associated with voids or "trees" in solid dielectrics, metal particles or sharp points on either solid conductors or insulators, or the presence of interfaces between different types of insulating or conducting media. Partial discharges are self-quenching because the surface or space charge produced during the discharge activity generally accumulates and acts to reduce the local electric-field magnitude to a level below which the discharge can be sustained.

Because of its self-quenching nature, the P-D phenomena is inherently pulsating and will manifest itself as current pulses in the external circuit. Partial-discharge activity occurs in regions that are at least partially in the gas phase such as occlusions in solids or "bubbles" formed by vaporization of a liquid. The partial-discharge phenomena 
that iccur around conductors in air or other gases is often referred to as corona. It shou'd be noted that corona discharges can, under some conditions, exhibit a non-pulsating characteristic for dc voltages which is associated with the formation of a steady glow region $[49,50]$. Because this type of corona discharge is steady and therefore not self-quenching, it does not fit into the category of P-D phenomena under consideration here.

Partial discharges can be observed by electrical, optical, or acoustical measurements that detect and record pulses. The determination of P-D pulse characteristics has been the subject of numerous previous investigations [51]. Techniques have been developed to measure iich parameters as mean P-D pulse amplitude and repetition rate [52], pulse-height distribution [53-55], pulse shapes [56], correlation of pulse amplitude with phase of an ac votage [57,59], and correlation between optical, electrical, or acoustical pulses. Because of the complex, stochastic nature of P$D$ phenomena, the results of such measurements have often been difficult, if not impossible, to interpret in terms of meaningful physical models. The measurement scheme discussed here is an exiension of a technique previously used to provide more refined information about the statistical characteristics of P-D pulses. From the additional information acquired by this method, it is possible to gain more insight into the physical bases of the phenomena which might prove useful in the design of P-D measurement systems of the future.

Partial discharge phenomena fit into a category of stochastic processes for which memory effects play an important role. The existence of memory is an inherent property of P-D phenomena because the occurrence of a discharge pulse is associated with the formation of residual ion space-charge, surface charges, and excited metastable species which can affect the initiation and growth of subsequent discharge pulses. As mentioned above, the pulsating characteristic of P-D's in various types of insulating media can be attributed to effects of space or surface charges. When the accumulation of discharge-generated space or surface charge causes the field to fall below a level sufficient to sustain the discharge, it ceases. Another P-D pulse will not occur at this site until the local field is restored such as can result from charge r.igration or changes in applied voltage.

Because of memory effects, correlations may exist among successive discharge pulses and measurement of these correlations can provide useful information on the nature of P-D phenomena. Recognition of this fact motivated the development of a system to extract information about memory effects. This system is described in a recent publication [60] and has been applied to an investigation of the stochastic behavior of Trichel-pulse, negative-corona discharges in electronegative gases $[52,61,62]$ and dielectric-barrier-type discharges in air which will be discussed in the next three sections [13].

It is convenient to represent the P-D phenomenon as a random point process [63] in which each pulse is identifed by a time $t_{n}$ and is "marked" with a particular property, 


\section{REPRESENTATION OF A MARKED RANDOM POINT PROCESS}

\begin{tabular}{|l|l|l|l|l|l|}
${ }^{a_{n-2}}$ & $\left.\right|^{a_{n-1}}$ & $a_{n}$ & $a_{n+1}$ & $a_{n+2}$ & \\
$\Delta t_{n-2}$ & $\Delta t_{n-1}$ & $\Delta t_{n}$ & $\Delta t_{n+1}$ & $\Delta t_{n+2}$ & $\Delta t_{n+3}$
\end{tabular}

Figure 26. Diagrammatic representation of the marked random point process corresponding to the Trichel-pulse discharge phenomenon. The $\Delta t_{n}$ are pulse time separations and the $q_{n}$ are pulse amplitudes.

in this case the pulse amplitude $q_{n}$, as indicated in figure 26 The stochastic process is thus defined by the set of ordered pairs $\left\{q_{n}, t_{n}\right\}, n=1,2,3, \ldots$ Because of memory effects, $q_{n}$ and $t_{n}$ can depend on the set $\left\{q_{n}, t_{n}\right\}_{j}$, for all $j<n$. The process can also be defined by the set $\left\{q_{1}, q_{n}, \Delta t_{n-1}\right\}_{j}, \quad n=2,3, \ldots, j$, where $\Delta t_{n-1}=t_{n}-t_{n-1}$, which is more appropriate to this discussion since it is the time separations between pulses that are either measured or specified by the measurement process. The pulse amplitude was chosen as the relevant mark because it is a measure of discharge intensity. In defining the point process, one could also consider other marks such as the pulse-shape parameters.

If memory is important in the P-D process, the variables $q_{n}, \Delta t_{n}, q_{n-1}$ and $\Delta t_{n-1}$ associated with adjacent events may not be independent. For example, the amplitude, $q_{n}$, may depend on the time separation, $\Delta t_{n-1}$. Indeed, this is found to be the case for Trichel-pulse discharges $[12,13,60-62,64]$. The dependence of $\Delta t_{n}$ on $q_{n}$ or $\Delta t_{n-1}$ is an indication of the influence of residuals from the previous pulse in either enhancing or retarding the initiation of the subsequent pulse. Likewise, the dependence $q_{n}$ on $\Delta t_{n-1}$ or $q_{n-1}$ is an indication of the influence of residuals from the previous pulse on the growth of the subsequent pulse.

Because of these dependencies, the unconditional pulse-amplitude and pulse timeseparation distributions $\left(p_{0}\left(q_{n}\right)\right.$ and $\left.p_{0}\left(\Delta t_{n}\right)\right)$ are not independent. If, for example, an external, radiation source is used to help initiate P-D's, $p_{0}\left(\Delta t_{n}\right)$ will change with the intensity of this source since the probability of discharge initiation changes with intensity. The changes in $p_{0}\left(\Delta t_{n}\right)$ will then be reflected in $p_{0}\left(q_{n}\right)$ if $q_{n}$ depends on $\Delta t_{n-1}$. Results from measurements of only one distribution, $p_{0}\left(q_{n}\right)$ or $p_{0}\left(\Delta t_{n}\right)$, as are obtained with most conventional P-D-measurement systems, will be difficult to interpret if memory effects are significant.

The ability to detect correlations among successive discharge pulses is a valuable diagnostic that could be incorporated into existing P-D measurement systems 


\section{BLOCK DIAGRAM OF THE STOCHASTIC MEASUREMENT SYSTEM}

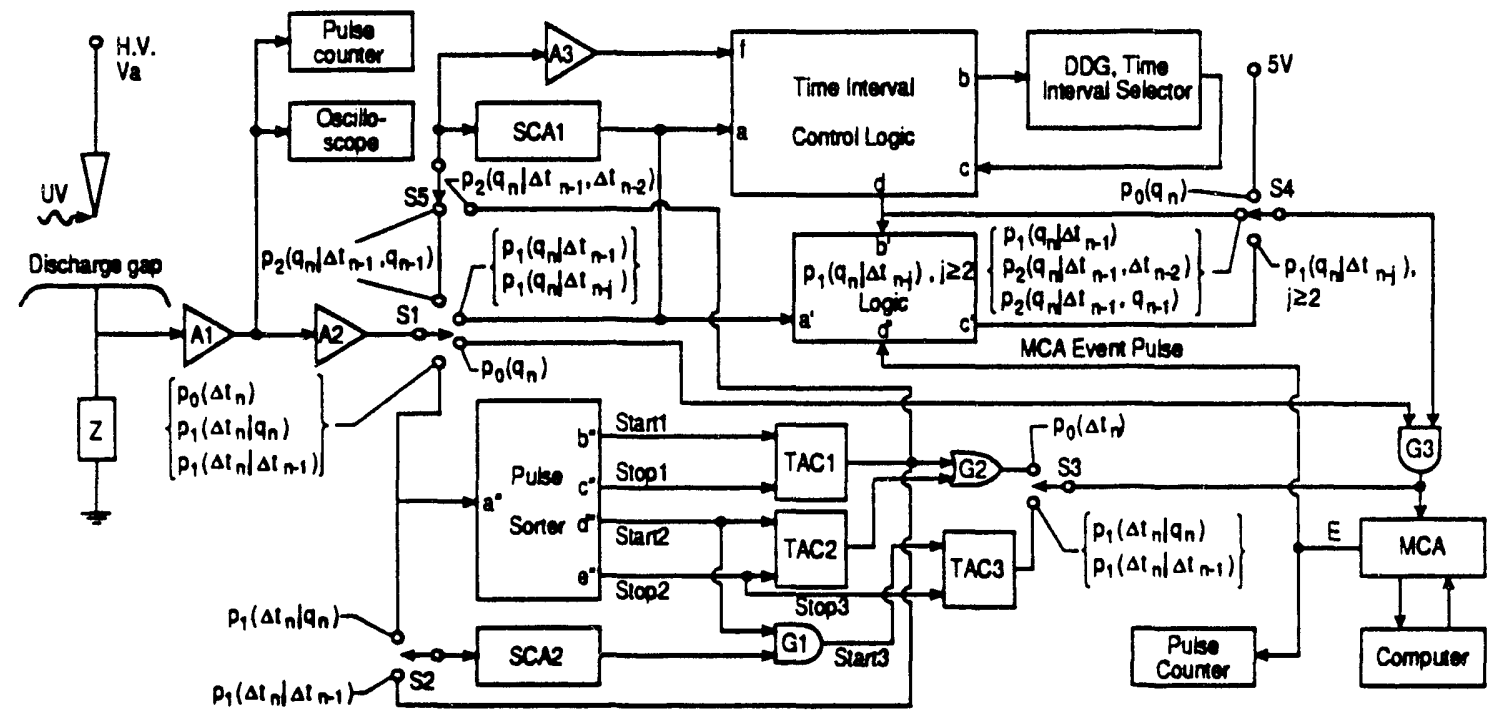

Figure 27. Diagrammatic representation of system for measuring pulse-amplitude and time-separation distributions. In this diagram, the relevant components are identified as follows: MCA - Multichannel analyzer; TAC - time-to-amplitude converter; SCA - single channel analyzer; DDG - digital-delay generator; A - amplifier; G - gate; and S - switch.

either directly through filtering techniques described here, or indirectly through off-line data analysis. The NIST system allows "real-time" measurement of a set of conditional pulse-amplitude and pulse-time-separation distributions, namely $p_{1}\left(q_{n} \mid q_{n-1}\right) ; p_{1}\left(q_{n} \mid \Delta t_{n-j}\right), j \geq 1 ; p_{1}\left(\Delta t_{n} \mid \xi\right), \xi=q_{n}$ or $\Delta t_{n-1} ;$ and $p_{2}\left(q_{n} \mid \Delta t_{n-1}, \xi\right)$, $\xi=q_{n-1}$ or $\Delta t_{n-2}$. These distributions are defined, for example, such that $p_{1}\left(q_{n} \mid \Delta t_{n-1}\right) d q_{n}$ is the probability that the $n$th P-D pulse has an amplitude in the range $q_{n}$ to $q_{n}+d q_{n}$ if it is separated from the previous pulse by a fixed time $\Delta t_{n-1}$, and $p_{2}\left(q_{n} \mid \Delta t_{n-1}, a_{n-1}\right) d q_{n}$ is the same but with both $\Delta t_{n-1}$ and $q_{n-1}$ fixed.

By comparison of the measured conditional and unconditional pulse-height and time-seperation distributions it is possible to infer immediately whether or not two variables are correlated and therefore dependent. If it is found. for example, that $p_{1}\left(q_{n} \mid \Delta t_{n-1}\right) \neq p_{0}\left(q_{n}\right)$ for at least some allowed values of $q_{n}$ and for all allowed $\Delta t_{n-1}$ such that $p_{0}\left(\Delta t_{n-1}\right) \neq 0$, then $q_{n}$ and $\Delta t_{n-1}$ are dependent variables. Similarly, if $p_{2}\left(q_{n} \mid \Delta t_{n-1}, q_{n-1}\right) \neq p_{1}\left(q_{n} \mid \Delta t_{n-1}\right)$ for allowed $q_{n-1}$, then a dependence must exist between $q_{n}$ and $q_{n-1}$ at a fixed $\Delta t_{n-1}$. One can also use the measured conditional distributions to compute correlation coefficients that provide a quantitative measure of the extent to which any two variables in the set $\left\{q_{1}, q_{n}, \Delta t_{n-1}\right\}_{j}$ are correlated.

Measurement of meaningful conditional distributions require the P-D process to be stationary, or at least quasistationary. If external factors that affect P-D behavior, such as the size of a void, or the electron emission properties of a surface, change furing the measurement, the data obtained for conditional distributions 
could give false indications of possible correlations. The extent to which external factors are important is perhaps best revealed by measurements of the unconditional distributions $p_{0}\left(q_{n}\right)$ and $p_{0}\left(\Delta t_{n}\right)$ which can be performed relatively more quickly than the measurements of conditional distributions. The Trichel-pulse corona discharge considered below is an example of a P-D phenomenon that is sufficiently stationary so as to allow measurement of all the conditional distributions for which the system is designed.

\subsubsection{Measurement System}

The modifications of the Stochastic Analyzer for Pulsating Phenomena (SAPP) originally described by Van Brunt and Kulkarni [60] have been made to: 1) allow direct real-time measurements of the conditional pulse-amplitude distributions $p_{1}\left(q_{n} \mid \Delta t_{n-j}\right), j>2$ and $\left.p_{2}\left(q_{n} \mid \Delta t_{n-1}, \Delta t_{n-2}\right) ; 2\right)$ eliminate intermediate pulse errors associated with measurement of the $p_{2}$ distributions; and 3 ) reduce background associated with accidental sampling of the tails of discharge pulses. A diagram of the measurement system is shown in figure 27. It should be noted that another version of this system has recently been constructed at NIST which can be applied to investigation of P-D's generated by ac voltages. This new system, which will be described later, allows measurement of distributions that are conditioned on a set of selectable phase intervals of the applied voltage.

The system in figure 27 may be configured to measure any of the indicated distributions by appropriate positioning of the switches (S1-S5). Data from all measurement configurations are accumulated by a 256-channel, computer-controlled multi-channel analyzer (MCA).

The system was first applied to an investigation of the stochastic behavior of negative corona (Trichel pulses) generated using a point-plane electrode gap configuration in electronegative gas mixtures [49]. The test gap is indicated in figure 27. The coronadischarge current pulses are detected electrically using a preamplifier (A1) connected to an impedance $Z$ that is in series with the discharge gap. The detected pulses are then sent to a variable gain amplifier (A2) before being routed to the appropriate circuit path. For measurement of $p_{0}\left(q_{n}\right)$, the discharge pulses from A2 are fed directly to the MCA through gate G3 which is kept open by proper positioning of S4.

The distribution $p_{0}\left(\Delta t_{n}\right)$ is measured by sending the pulses from $A 2$ to a pulse sorter used to trigger two time-to-amplitude converters (TAC1 and TAC2) which generate output pulses of amplitude proportional to the time between P-D pulses. The outputs of TAC1 and TAC2 are fed to the MCA through gate G2 by appropriate positioning of S3. As shown in ref. [60], this arrangement permits the recording of every sequential time interval, provided all time separations exceed the TAC reset time of $50 \mu \mathrm{s}$. 
The measurement of $p_{1}\left(q_{n} \mid \Delta t_{n-1}\right)$ requires use of a logic-controlled digital-delay generator (DDG) to restrict the transfer of pulses from A2 to the MCA through G3 to a narrow preselected time interval $\Delta t_{n-1} \pm \delta\left(\Delta t_{n-1}\right) / 2$ for which $\Delta t_{n-1}>\delta\left(\Delta t_{n-1}\right)$. To measure $p_{2}\left(q_{n} \mid \Delta t_{n-1}, q_{n-1}\right)$, a single-channel analyzer (SCA1) is inserted between A2 and the input to the DDG time-interval control logic circuit by proper positionings of $\mathrm{S} 1$ and S5. The SCA1 restricts triggering of the DDG to pulses having amplitudes within the selected range $q_{n-1} \pm \delta\left(q_{n-1}\right) / 2$ for which $q_{n-1}>>\left(q_{n-1}\right)$ [60]. To insure, in the case of $p_{2}$ measurements, that the time-interval control logic properly inhibits the opening of G3 if intermediate pulses occur before the selected time window, it is necessary to modify the circuit previously described [60] by adding the amplifier A3 with input at $f$. This amplifier is identical to that defined by transistor $T_{1}$ in figure 16 of ref. [60]. In the present configuration, the input $f$ is connected directly to the delay $\tau_{1}$ of figure 16 (ref. [60]) which has, in turn, been disconnected from $T_{1}$.

Unlike the system previously described [60], the present system allows direct measurement of $p_{2}\left(q_{n} \mid \Delta t_{n-1}, \Delta t_{n-2}\right)$. For measurement of this distribution, the switches $\mathrm{S} 1$ and $\mathrm{S} 5$ are positioned so that the input to SCA1 is derived from TAC1. The time interval measured by $\mathrm{TACl}$ is restricted by SCA1 to lie within a narrow range $\Delta t_{n-2} \pm \delta\left(\Delta t_{n-2}\right) / 2$ for triggering the DDG, which, in turn, defines $\Delta t_{n-1}$.

By the indicated positionings of switches $\mathrm{S} 1, \mathrm{~S} 2$, and $\mathrm{S} 5$, it is possible to measure the conditional pulse time-separation distributions $p_{1}\left(\Delta t_{n} \mid \Delta t_{n-1}\right)$ and $p_{1}\left(\Delta t_{n} \mid q_{n}\right)$. For measurement of $p_{1}\left(\Delta t_{n} \mid \Delta t_{n-1}\right)$, the output of TAC1 is fed to the input of the singlechannel analyzer SCA2 which thus defines $\Delta t_{n-1}$ and triggers TAC3 for measurement of the next time interval $\Delta t_{n}$. In the case of the $p_{1}\left(\Delta t_{n} \mid q_{n}\right)$ measurement, SCA2 is connected directly to amplifier A2 thereby used to define $q_{n}$.

The present system also differs from that previously described in that the $p_{1}\left(q_{n} \mid \Delta t_{n-j}\right)$ logic circuit now contains a pulse counter that can be set for any $j \geq 2$. By adjustment of the delays inherent to the time-interval control logic and with minor adjustments in the shape of the gate pulse from this circuit, it has been possible to largely eliminate the background problem illustrated by figure 19 of ref. [60].

In the present measurement system, the pulse detection-circuit conditions are similar to those described in ref. [60] so that the measured discharge-pulse amplitude is proportional to the net charge transported in the discharge given by

$$
Q_{n}^{\prime}=\int_{-\infty}^{\infty} i_{n}(t) d t,
$$

where $i_{n}(t)$ is the instantaneous discharge current at time $t$ for the $n$th pulse. Given the impulse response $h\left(t-t^{\prime}\right)$ of the detection circuit (defined here mainly by the filter characteristics of $\mathrm{Z}$ and $\mathrm{A} 1$ ), the observed pulse signal is given by

$$
q_{n}^{\prime}(t)=\int_{-\infty}^{t} i_{n}\left(t^{\prime}\right) h\left(t-t^{\prime}\right) d t^{\prime} .
$$


The impulse response in the present measurement system has a width, $w_{i}$, of about $1.5 \mu \mathrm{s}$ whereas the intrinsic width, $w_{p}$, of a typical Trichel pulse is known [56] to be on the order 10 to $50 \mathrm{~ns}$. Therefore the condition $w_{i}>>w_{p}$ allows the approximation

$$
i_{n}(t) \simeq Q_{n}^{\prime} \delta\left(t-t_{n}\right),
$$

where $\delta\left(t-t_{n}\right)$ is the Dirac delta function. Using this in equation(7) gives

$$
q_{n}^{\prime}(t)=Q_{n}^{\prime} h\left(t-t_{n}\right)
$$

This means that the shapes of the observed Trichel pulses in the present experiment are governed primarily by the shape of $h\left(t-t_{n}\right)$ and thus the maximum of $q_{n}^{\prime}(t)$. denoted here by $q_{n}$, is directly proportional to $Q_{n}^{\prime}$. Therefore, $q_{n}$ can be expressed in units of charge, and in the present case, it is convenient to use picocoulombs ( $\mathrm{pC}$ ). The method for calibrating the pulse-height measurements in terms of charge is the same as described previously [55].

\subsubsection{Examples of Results from Investigation of Trichel Pulses}

The Trichel-pulse discharge [65] was selected for initial test of the SAPP measurement . concept because its properties are reasonably well understood [66-70] and it can be easily generated and controlled in a simple point-plane gap. With some care, the Trichel-pulse discharge can be maintained in a stable condition for relatively long periods of time. The phenomenon is shown [12] to be a clear example of a non-Markovian, marked random point process in which memory effects play an important role. Strong correlations are found to exist among the amplitudes and time separations of successive discharge pulses which can be interpreted in terms of the influences of ion space charge and metastable species from previous pulses on the initiation and growth of subsequent pulses.

As an example of the richness of information that can be extracted with the present measurement system, results obtained for self-sustained Trichel pulses in a neonoxygen gas mixture are shown in figures 28-32. Data for the different sets of distributions were obtained at different times so that the cathode surface conditions that apply, for example, to the results in figure 28 differ slightly from those that apply to figure 29. This accounts for the difference in $p_{0}\left(q_{n}\right)$ shown in these two figures. A detailed interpretation of the results presented here would go beyond the scope of this report. However, salient features of the data and certain important conclusions that can be derived therefrom should be noted. A more complete discussion of the physical bases for the stochastic behavior of the Trichel-pulse phenomenon has been given by Van Brunt and Kulkarni [12].

The fact that the second-order conditional distributions, $p_{2}$, differ from the corresponding first-order distributions, $p_{1}$, which in turn differ from the corresponding unconditional distributions, $p_{0}$, indicates unequivocally that the set of variables 
CONDITIONAL AND UNCONDITIONAL PULSE-AMPLITUDE DISTRIBUTIONS

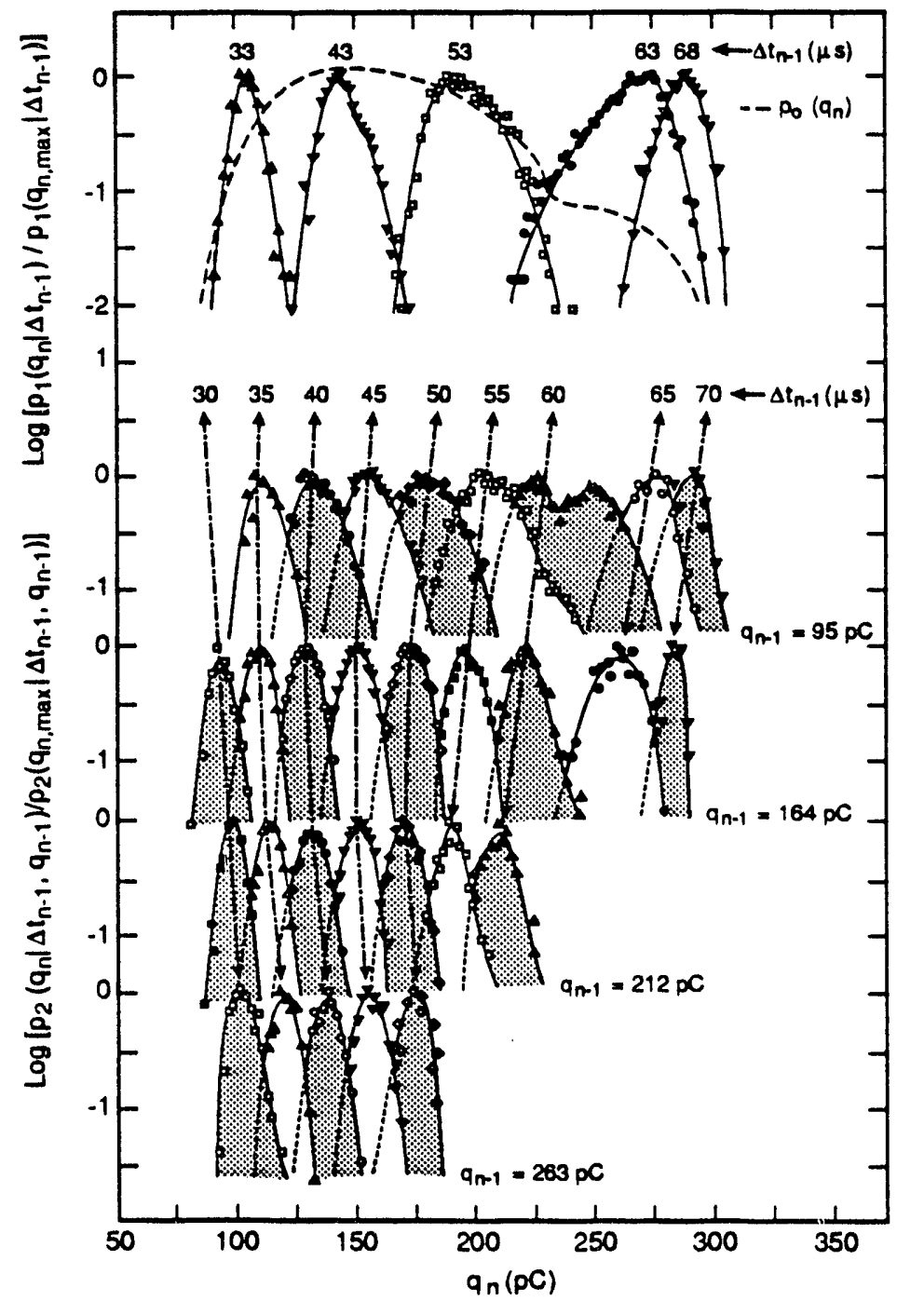

Figure 28. Measured unconditional and conditional discharge pulse-amplitude distributions $p_{0}\left(q_{n}\right), p_{1}\left(q_{n} \mid \Delta t_{n-1}\right)$, and $p_{2}\left(q_{n} \mid \Delta t_{n-1}, q_{n-1}\right)$ for the indicated fixed values for $\Delta t_{n-1}$ and $q_{n-1}$. The distributions have been normalized to the maxima. These results are for negative-corona pulses in a neon-oxygen/gas mixture. 


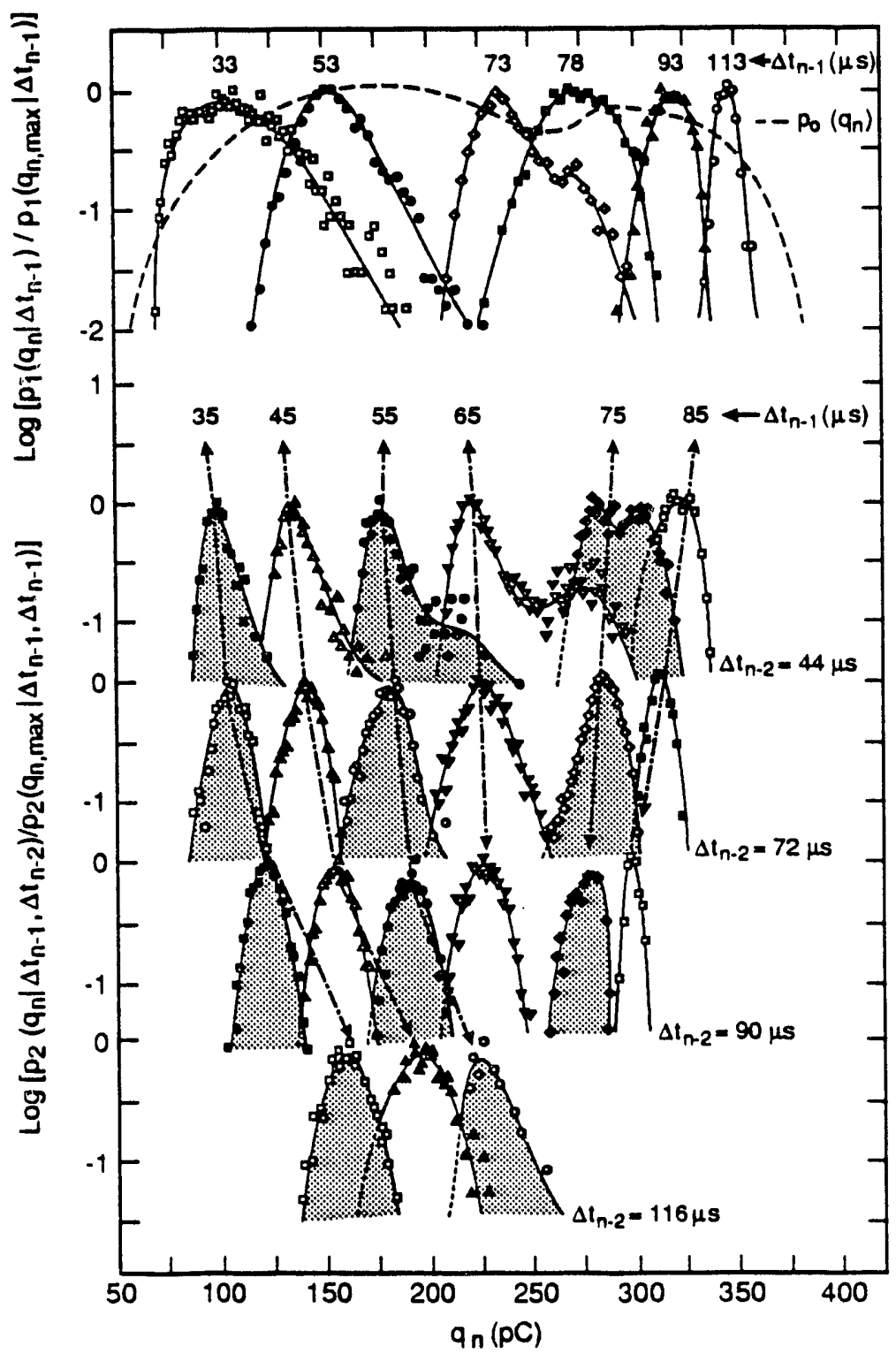

Figure 29. Measured unconditional and conditional discharge pulse-arrplitude distributions $p_{0}\left(q_{n}\right), p_{1}\left(q_{n} \mid \Delta t_{n-1}\right)$, and $p_{2}\left(q_{n} \mid \Delta t_{n-1}, \Delta t_{n-2}\right)$ for the indicated fixed values for $\Delta t_{n-1}$ and $\Delta t_{n-2}$. The distributions have been normalized to the maxima. These results are for negative-corona pulses in a neon-oxygen/gas mixture. 


\section{CONDITIONAL AND UNCONDITIONAL PULSE}

TIME-SEPARATION DISTRIBUTIONS

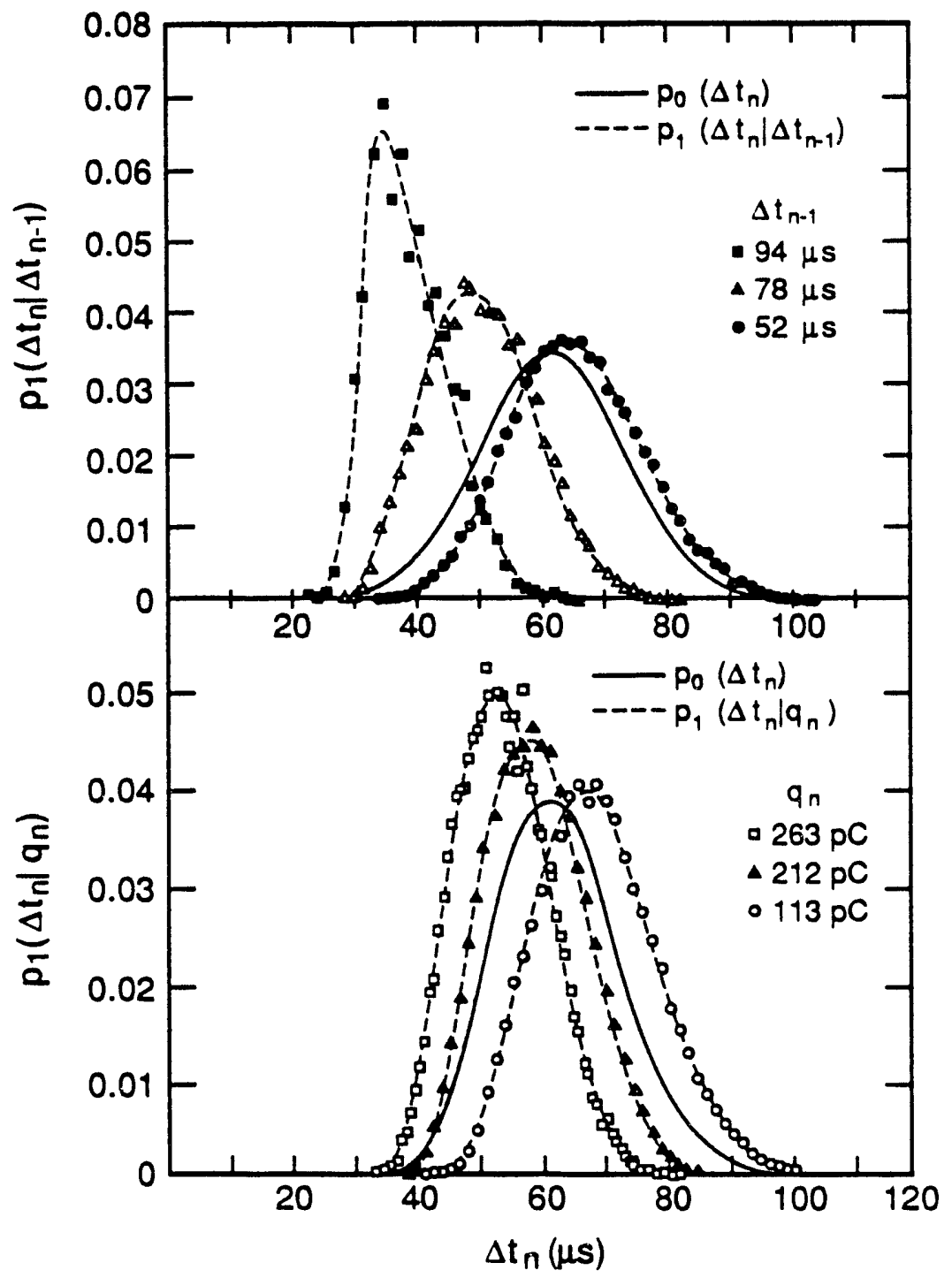

Figure 30. Measured unconditional and conditional discharge pulse-time-separation distributions $p_{0}\left(\Delta t_{n}\right), p_{1}\left(\Delta t_{n} \mid q_{n}\right)$, and $p_{1}\left(\Delta t_{n} \mid \Delta t_{n-1}\right)$ for the indicated fixed values for $q_{n}$ and $\Delta t_{n-1}$. The distributions have been normalized to the areas under the curves. These results are for negative-corona pulses in a neon-oxygen/gas mixture. 


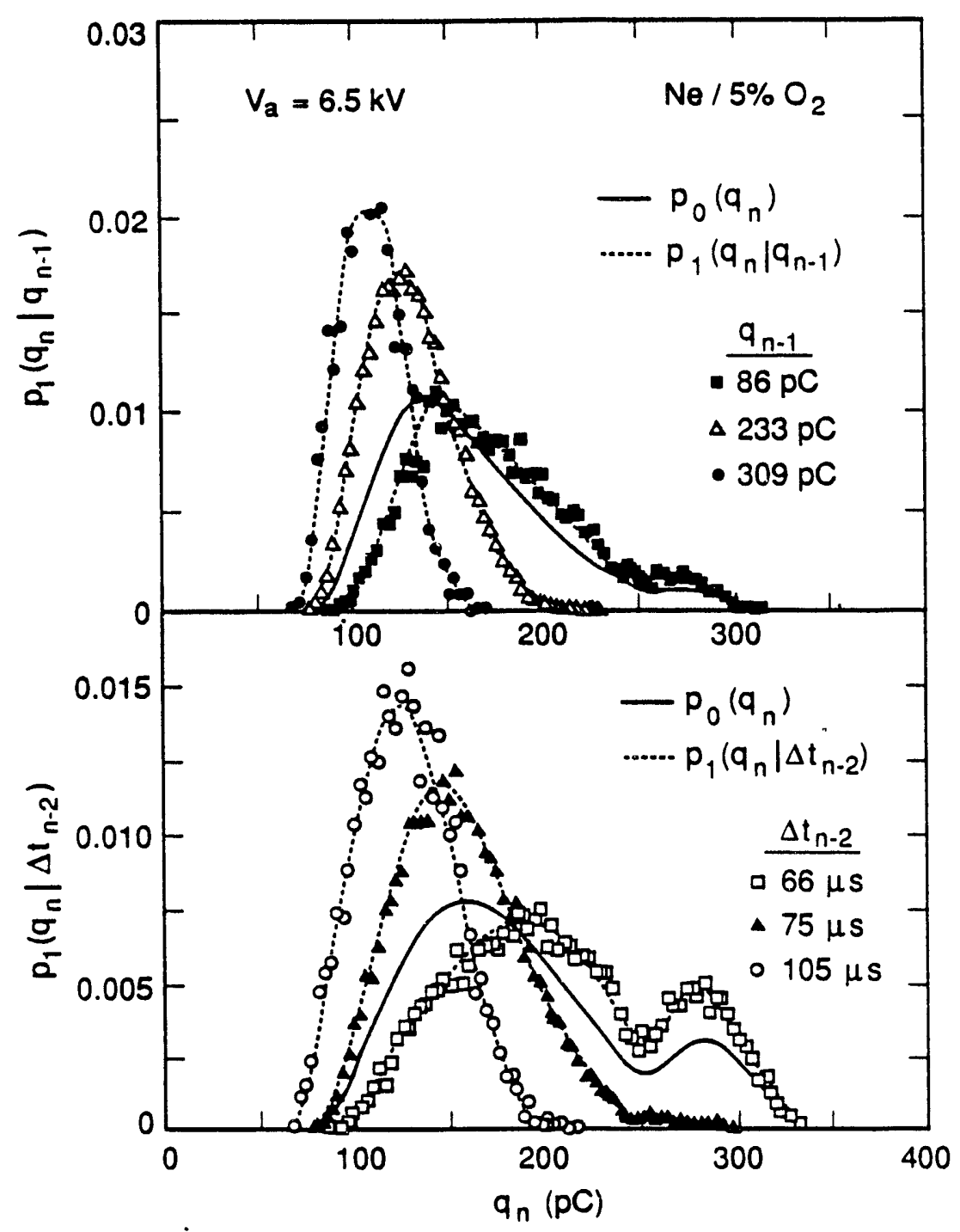

Figure 31. Measured unconditional and conditional discharge pulse-amplitude distributions $p_{0}\left(q_{n}\right), p_{1}\left(q_{n} \mid \Delta t_{n-2}\right)$, and $p_{1}\left(q_{n} \mid q_{n-1}\right)$ for the indicated fixed values for $\Delta t_{n-2}$ and $q_{n-1}$. The distributions have been normalized to the areas under the curves. These results are for negative-corona pulses in a neon-oxygen/gas mixture. 


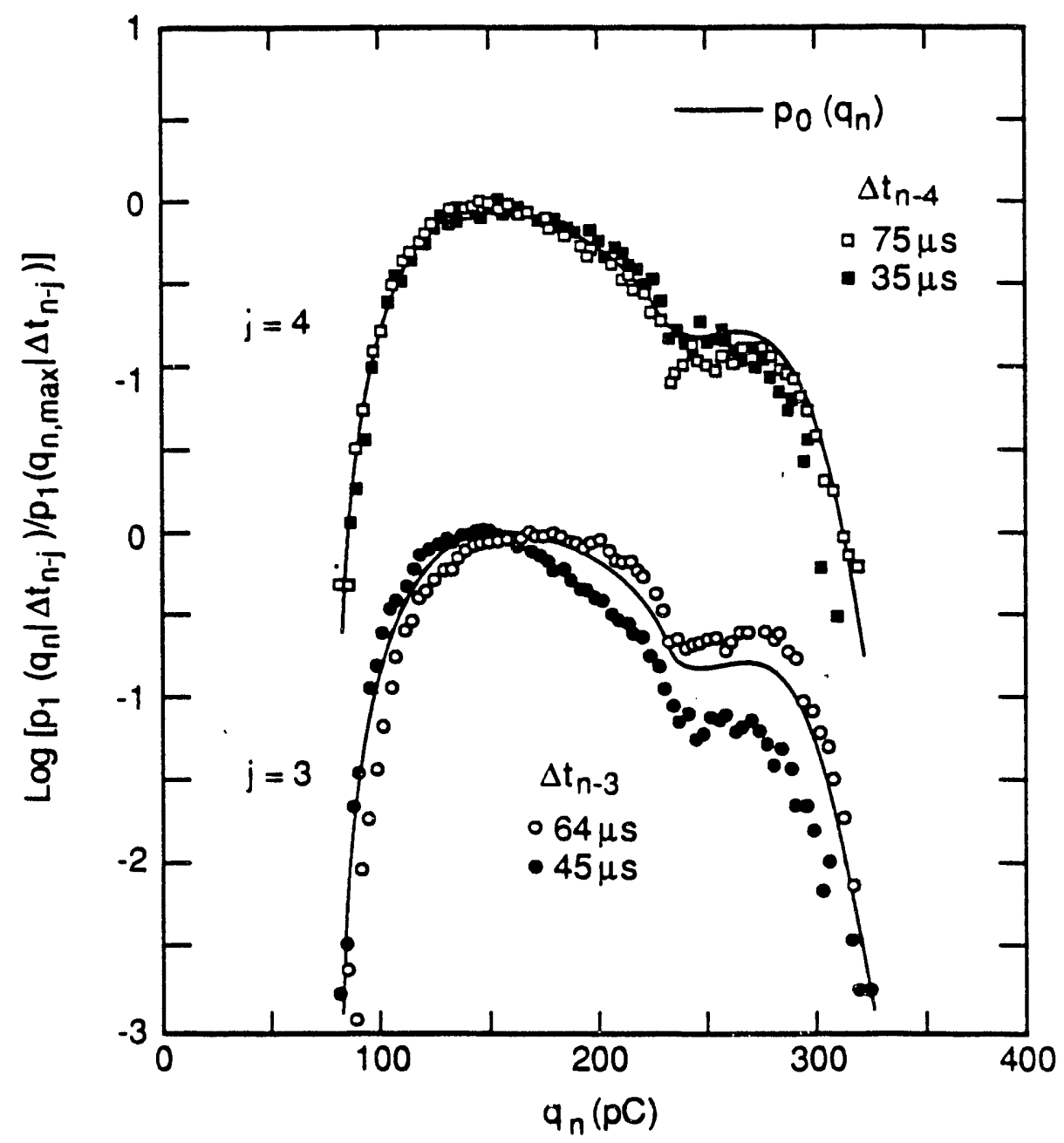

Figure 32. Measured unconditional and conditional discharge pulse-amplitude distributions $p_{0}\left(q_{n}\right), p_{1}\left(q_{n} \mid \Delta t_{n-3}\right)$, and $p_{1}\left(q_{n} \mid \Delta t_{n-4}\right)$ for the indicated fixed values for $\Delta t_{n-3}$ and $\Delta t_{n-4}$. The distributions have been normalized to the maxima. These results are for negative-corona pulses in a neon-oxygen/gas mixture. 
$\left\{\Delta t_{n}, q_{n}, \Delta t_{n-1}, q_{n-1}, \Delta t_{n-2}, \cdots\right\}$ associated with adjacent pulses are not independent. For example, it is seen from figures 28 and 29 that $q_{n}$ has a strong positive dependence on $\Delta t_{n-1}$. This dependence can be related to the influence of negative-ion space charge from previous pulses in suppressing the magnitude of the electric field at the cathode when the next pulse develops. It is also seen from figure 28 that the amplitude, $q_{n}$, of a pulse can be either positively or negatively dependent on the amplitude of the previous pulse. The sign of this dependence can be explained in terms of the competing effects of negative-ion space charge and metastable species in respectively retarding or enhancing the growth of the next pulse. The negative dependence of $\Delta t_{n}$ on $q_{n}$ (and $\Delta t_{n-1}$ ) implied by the conditional time-separation distributions shown in figure 30 can be understood in terms of the influence of metastable species from the previous pulse in enhancing the probability for initiating the next pulse by ejecting electrons from the cathode surface during field-assisted quenching.

Because of the correlations among the amplitudes and time separations of successive pulses, the distributions shown in figures 28-30 are all related. It can be shown, for example, from the law of probabilities that $p_{0}\left(q_{n}\right), p_{0}\left(\Delta t_{n}\right)$, and $p_{1}\left(q_{n} \mid \Delta t_{n-1}\right)$ are related by the integral expression

$$
p_{0}\left(q_{n}\right)=\int_{0}^{\infty} p_{0}\left(\Delta t_{n-1}\right) p_{1}\left(q_{n} \mid \Delta t_{n-1}\right) d\left(\Delta t_{n-1}\right)
$$

and the distributions $p_{0}\left(q_{n}\right), p_{0}\left(\Delta t_{n}\right), p_{1}\left(q_{n} \mid \Delta t_{n-1}\right), p_{1}\left(\Delta t_{n} \mid q_{n}\right)$, and $p_{2}\left(q_{n} \mid q_{n}, \Delta t_{n-1}\right)$ are related by

$$
p_{0}\left(\Delta t_{n-1}\right) p_{1}\left(q_{n} \mid \Delta t_{n-1}\right)=\int_{0}^{\infty} p_{0}\left(q_{n-1}\right) p_{1}\left(\Delta t_{n-1} \mid q_{n-1}\right) p_{2}\left(q_{n} \mid q_{n-1}, \Delta t_{n-1}\right) d q_{n-1} .
$$

Equation (10) indicates that if $q_{n}$ is dependent on $\Delta t_{n-1}$, then any externallyinduced change in the time-interval distribution, $p_{0}\left(\Delta t_{n}\right)$, will necessarily change the amplitude distribution, $p_{0}\left(q_{n}\right)$.

Since, as seen from the data in figures 29,31 , and, 32, the profiles for $p_{2}\left(q_{n} \mid \Delta t_{n-1}, \Delta t_{n-2}\right)$ and for $p_{1}\left(q_{n} \mid \Delta t_{n-j}\right), j=2,3,4$ do not match the profile for $p_{0}\left(q_{n}\right)$, it can be stated that $q_{n}$ depends on $\Delta t_{n-j}, j>1$, and therefore, the process is one for which memory extends back in time beyond the most recent event, i.e., the process is non-Markovian. This observation is consistent with results reported in the recent work of Steiner [64]. It can, in fact, be shown [12] that because of the relatively strong dependence of $q_{n}$ on both $\Delta t_{n-1}$ and $q_{n-1}$, it is possible for memory to propagate indefinitely back in time. 


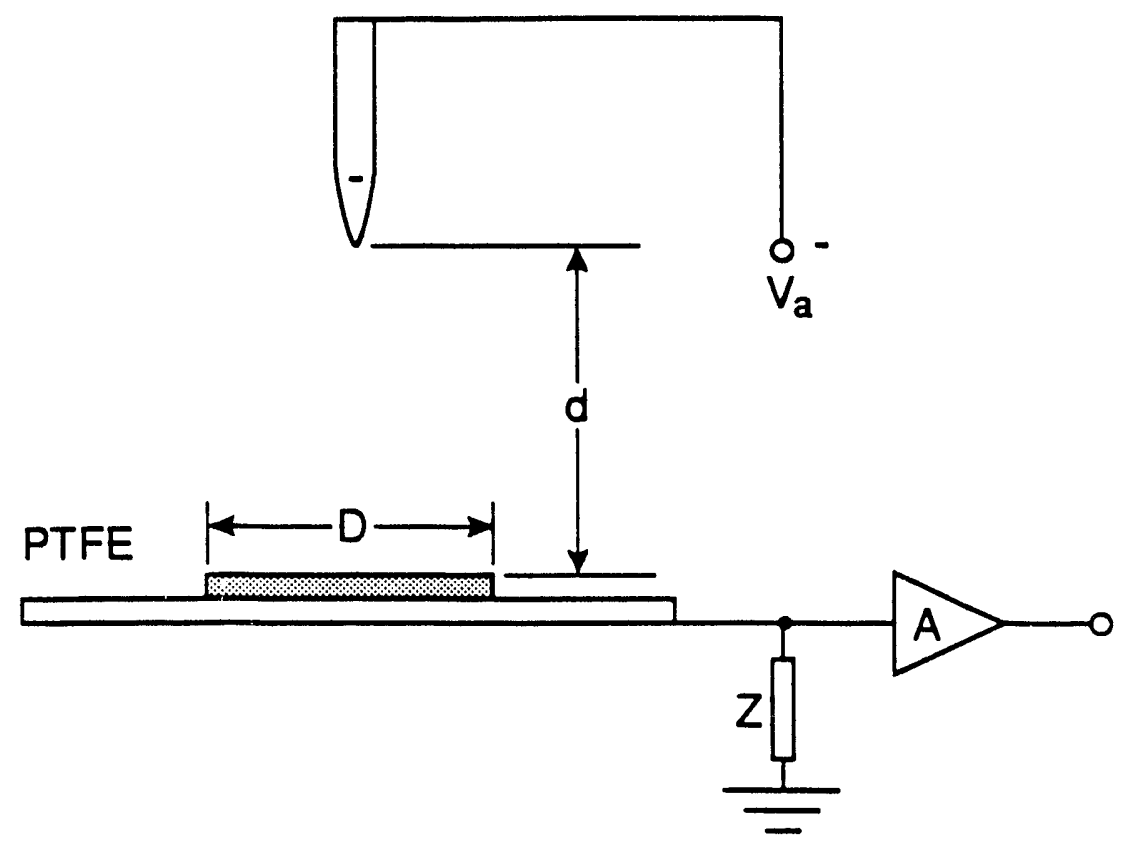

Figure 33. Electrode configuration.

\subsubsection{Influence of a Dielectric Barrier on the Stochastic Behavior of Trichel-Pulse Corona}

The investigation discussed here was undertaken to examine the influence that a thin solid polytetrafluoroethylene (PTFE) dielectric barrier has on the stochastic behavior of negative (Trichel) pulse corona discharges in air. Although the effects of solid dielectrics on corona pulses have been examined in previous experiments [71], there were no attempts to characterize the stochastic behavior of the phenomenon in these investigations. It is shown here from the measurements of conditional pulse-amplitude distributions that corona-induced charging of dielectric barriers placed on the planar electrode introduces another memory effect that becomes increasingly important as the point-to-plane gap spacing is reduced. It is also shown that, because the PTFE dielectric acquires a "quasi-permanent" charge from the corona, there is a critical gap spacing below which the Trichel-pulses appear to cease.

The experiments were carried out using the SAPP technique described above. The electrode-gap configuration used to obtain the results presented here is shown in figure 33. A PTFE dielectric of $1.0 \mathrm{~mm}$ thickness and of diameter $D$ was placed on the plane electrode and centered on the point-to-plane axis. The point-to-plane spacing, $d$, could be varied from 0 to $10 \mathrm{~cm}$. A very sharp stainless-steel point electrode with a radius of curvature at the tip of less than $0.01 \mathrm{~mm}$ was used to insure that, at a sufficiently high gap voltage, $V_{a}$, the discharge could be sustained by field-induced electron emission. The measurements described here were performed using "room air" in the gap. The corona-discharge current pulses were detected electrically using 


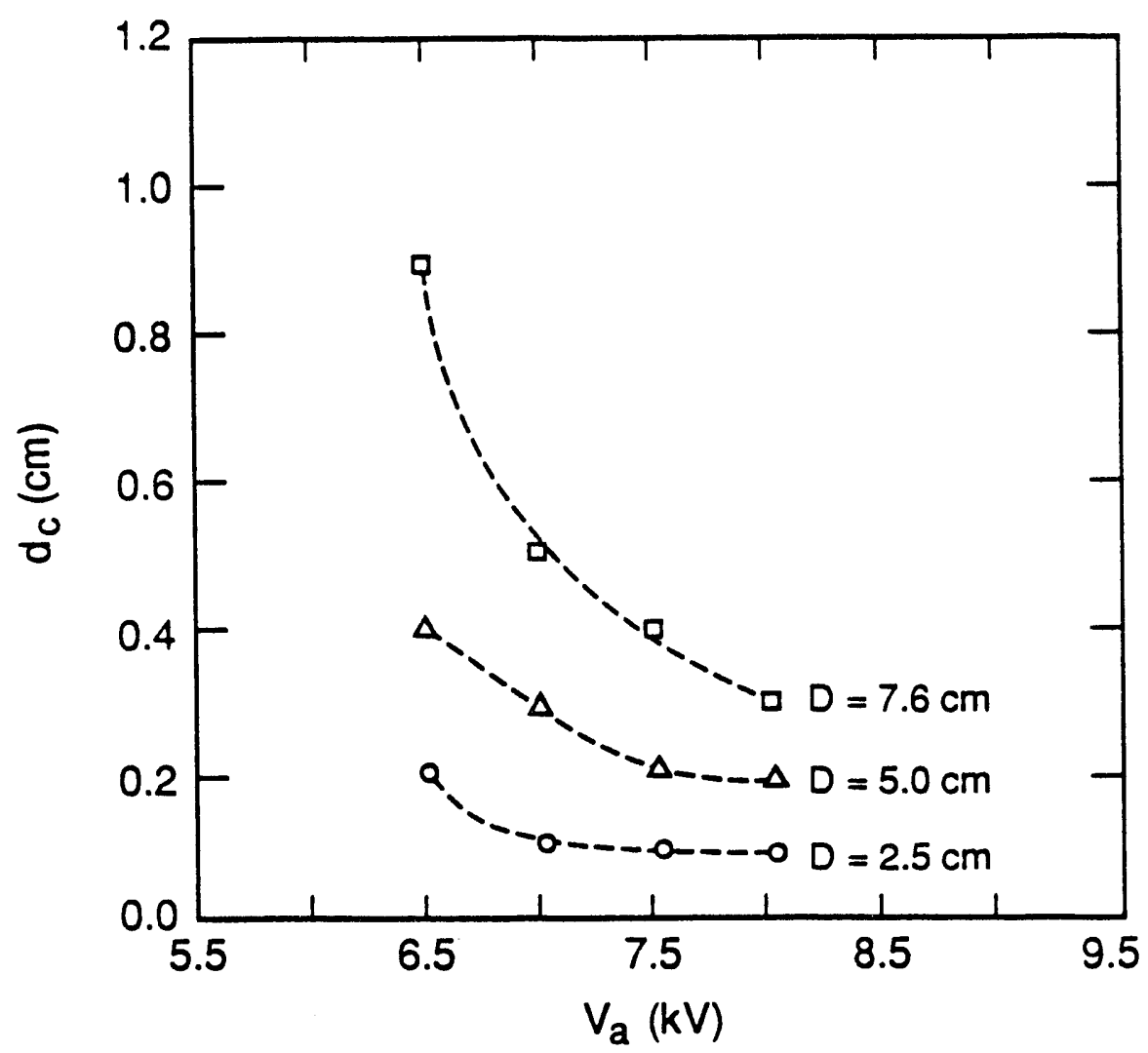

Figure 34. The measured critical point-to-plane gap spacing, $d_{c}$ below which the Trichel-pulse discharge ceases as a function of applied gap voltage for various indicated diameters of the PTFE insulator.

a preamplifier, $A$, connected to an impedance, $Z$, in series with the discharge gap as shown. The output of $A$ was fed to the system shown in figure 27

The system was used here to measure the unconditional pulse-height and pulse timeseparation distributions, $p_{0}\left(q_{n}\right)$ and $p_{0}\left(\Delta t_{n}\right)$ respectively, and the conditional pulseheight distribution $p_{1}\left(q_{n} \mid \Delta t_{n-1}\right)$.

It is found for the configuration shown in figure 33 that there is a critical point-toplane spacing below which it is not possible to observe a recognizable continuous Trichel-pulse discharge behavior. Figure 34 shows the measured critical point-toplane gap separations, $d_{c}$, at which the Trichel-pulse discharge ceases for different indicated values of the diameter $D$. A pulsating discharge may still occur for $d<d_{c}$, but the time separation between pulses becomes very long (many seconds). It is seen that the larger the diameter of the dielectric, $D$, and the lower the applied voltage, $V_{a}$, the larger will be the critical distance $d_{c}$ at which the corona extinguishes.

Examples of measured $p_{0}\left(q_{n}\right)$ and $p_{0}\left(\Delta t_{n}\right)$ at different indicated gap distances $d$ are shown in figures 35 and 36 respectively for the conditions $V_{a}=6.5 \mathrm{kV}, D=$ $5.0 \mathrm{~cm}$ and $V_{a}=10.1 \mathrm{kV}, D=7.5 \mathrm{~cm}$. For convenience in plotting, all of the 


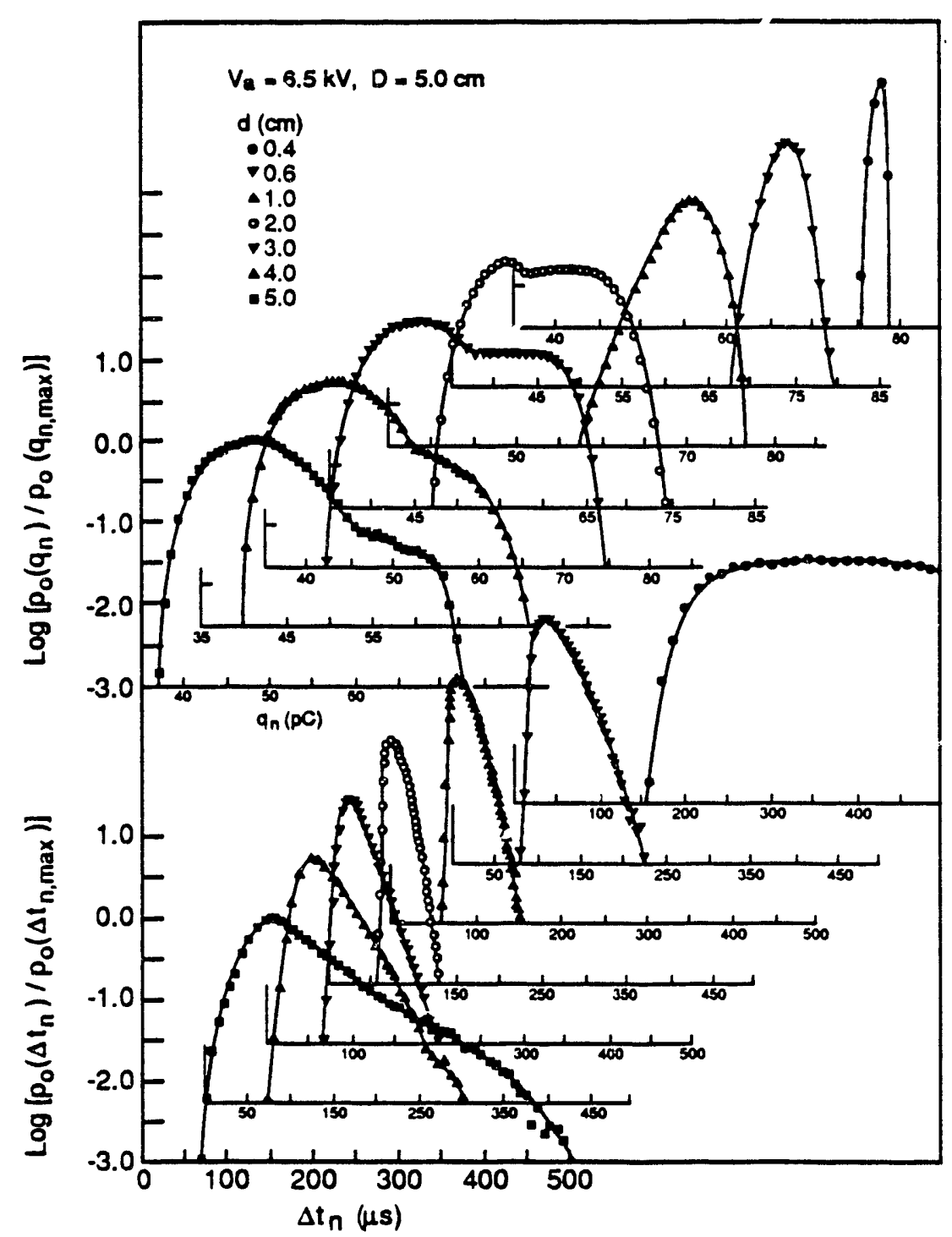

Figure 35. Measured pulse-height and corresponding time-intervai distributions at $V_{a}=6.5 \mathrm{kV}, D=5.0 \mathrm{~cm}$, for the various indicated gap spacings. The distributions have been normalized to the maxima. 


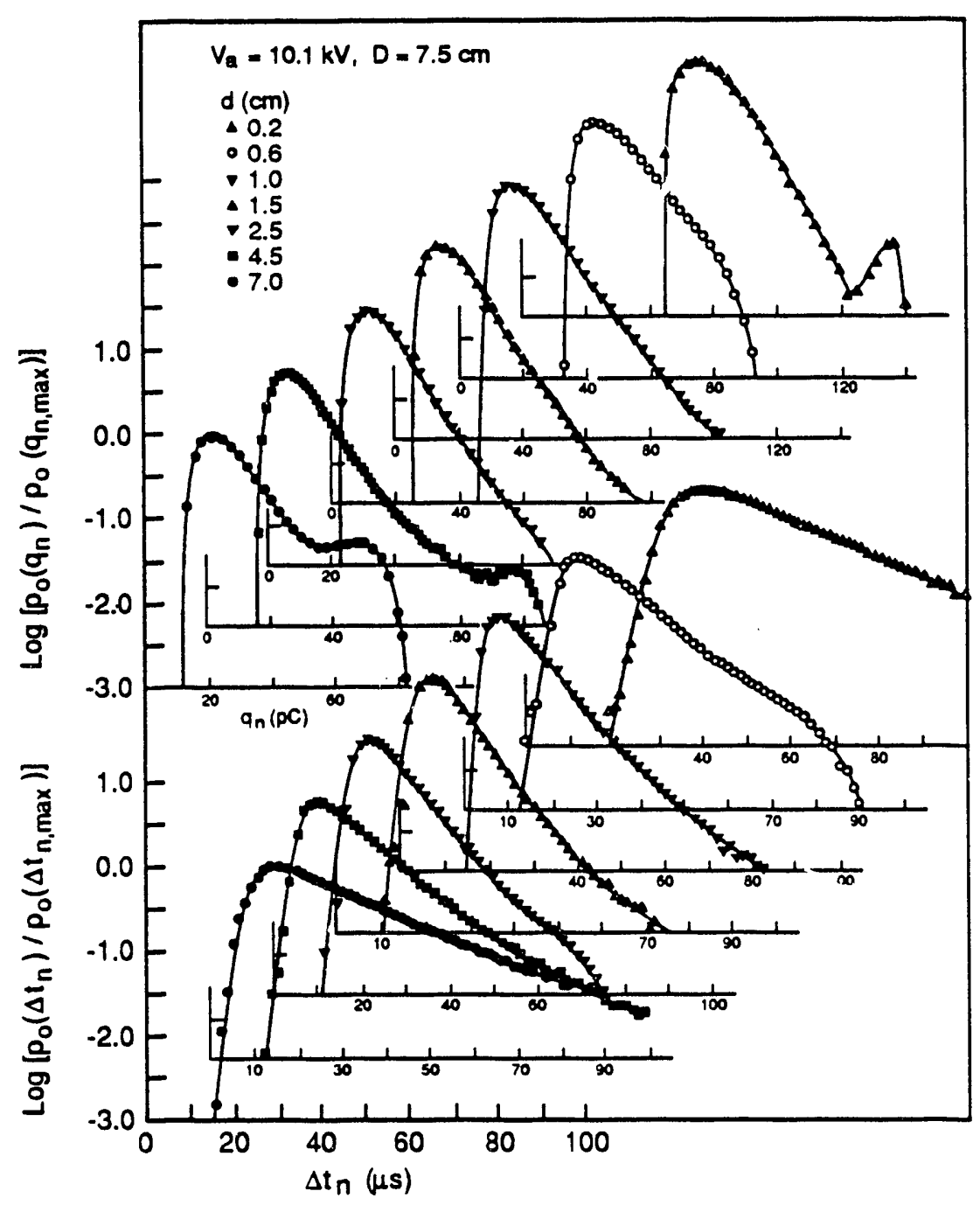

Figure 36. Measured pulse-height and corresponding time-interval distributions at $V_{a}=10.1 \mathrm{kV}, D=7.5 \mathrm{~cm}$, for the various indicated gap spacings. The distributions have been normalized to the maxima. 


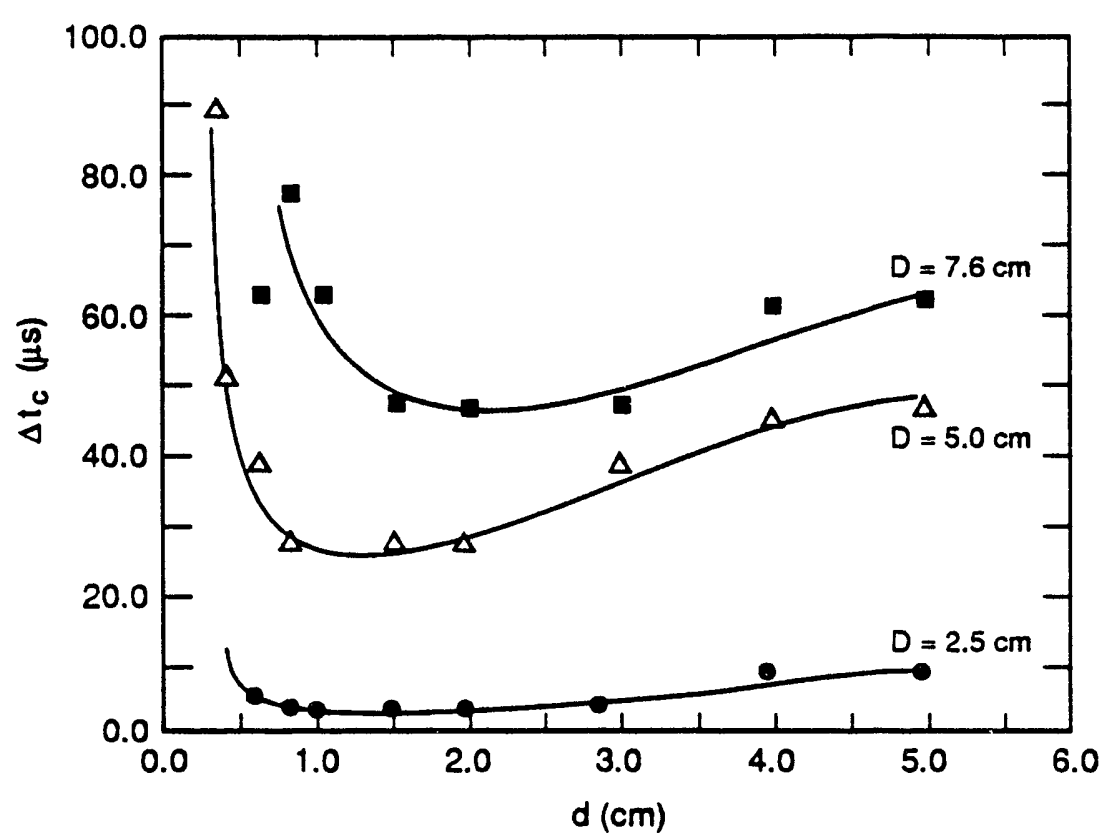

Figure 37. Minimum possible pulse time separation, $\Delta t_{c}$, versus gap spacing for $V_{a}=7.5 \mathrm{kV}$ and the different indicated PTFE diameters.

distributions shown in these figures have been arbitrarily normalized to the maximum values. The profiles of the distributions shown here exhibit features similar to those previously reported and explained by Van Brunt and Kulkarni [12]. For example, the distributions $p_{0}\left(\Delta t_{n}\right)$ indicate a previously observed [12,72] critical minimum time separation, $\Delta t_{c}$, between successive pulses. The rate of decrease in $p_{0}\left(\Delta t_{n}\right)$ for $\Delta t_{n}>\Delta t_{n, \max }$, where $\Delta t_{n, \max }$ is the maximum in $p_{0}\left(\Delta t_{n}\right)$, is controlled by the rate of electron emission at the point electrode [12] and is thus strongly dependent on the local electric field. It is known that $\Delta t_{c}$, which is controlled by the ambipolar diffusion of ions away from the point, also decreases exponentially with the field near the cathode. Examples of results for $\Delta t_{c}$ versus $d$ at different indicated values of $D$ are shown in figure 37. The fact that both $\Delta t_{c}$ and the width of $p_{0}\left(\Delta t_{n}\right)$ go through a minimum at a particular $d>d_{c}$ indicates that the electric field at the cathode goes through a maximum at this separation. This maximum in the field does not occur when the dielectric is removed as illustrated by the measured data for $p_{0}\left(q_{n}\right)$ and $p_{0}\left(\Delta t_{n}\right)$ shown in figure 38 that were obtained under conditions where no dielectric was present on the planar electrode. The corresponding $\Delta t_{c}$ extracted from these data are plotted in figure 39 and show the expected monotonic decrease with decreasing $d$ which, for a fixed $V_{a}$, is consistent with an increasing field strength at the point electrode.

The profiles of the pulse-height distributions, $p_{0}\left(q_{n}\right)$, are generally consistent with equation(6) and thus can be explained in terms of the physical processes that determine the profile of the corresponding $p_{0}\left(\Delta t_{n}\right)$ distribution coupled with the 


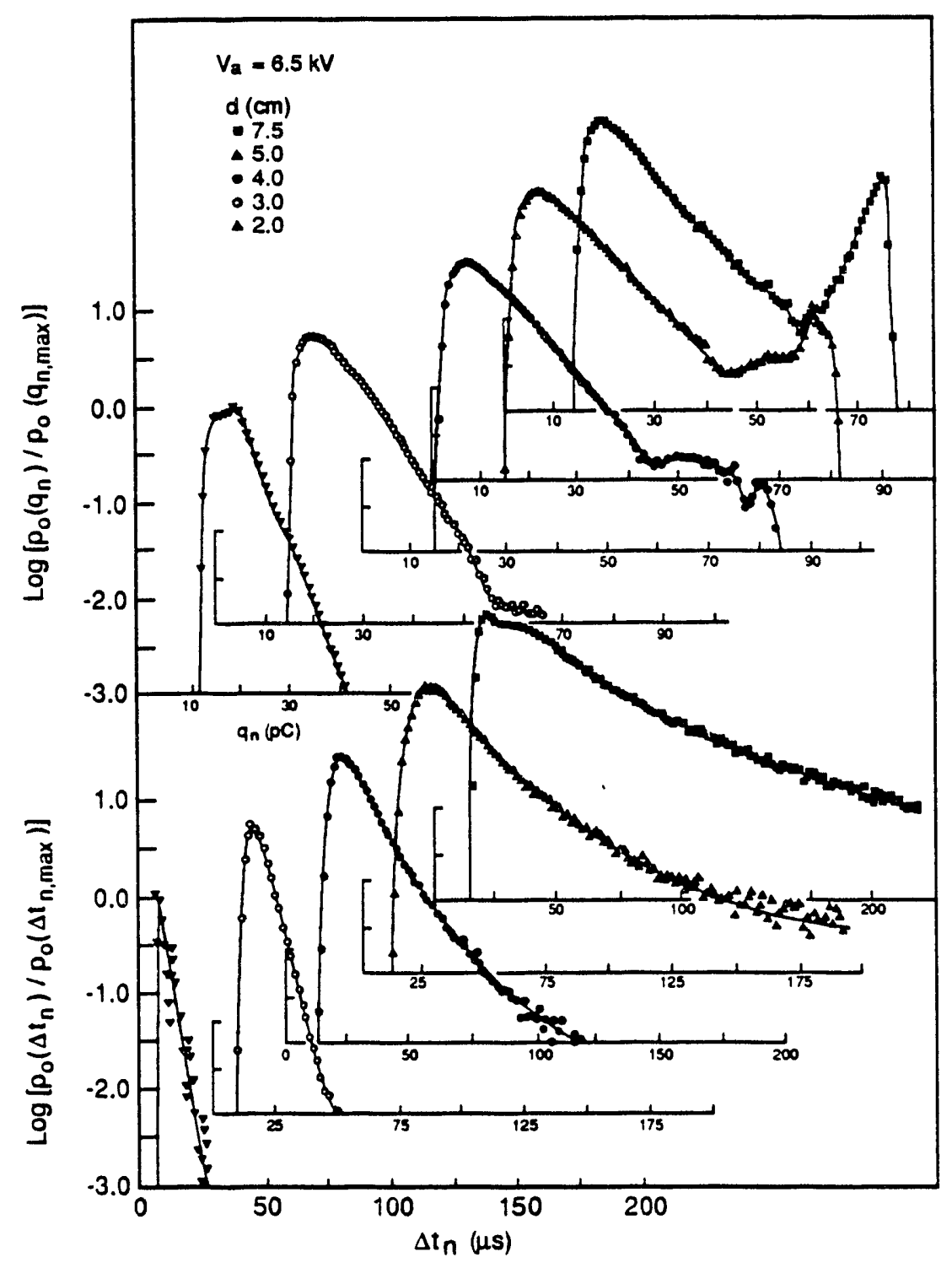

Figure 38. Measured pulse-height and corresponding time-interval distributions at $V_{a}=6.5 \mathrm{kV}$ for various indicated gap spacings when no dielectric is on the anode surface. The distributions have been normalized to the maxima. 


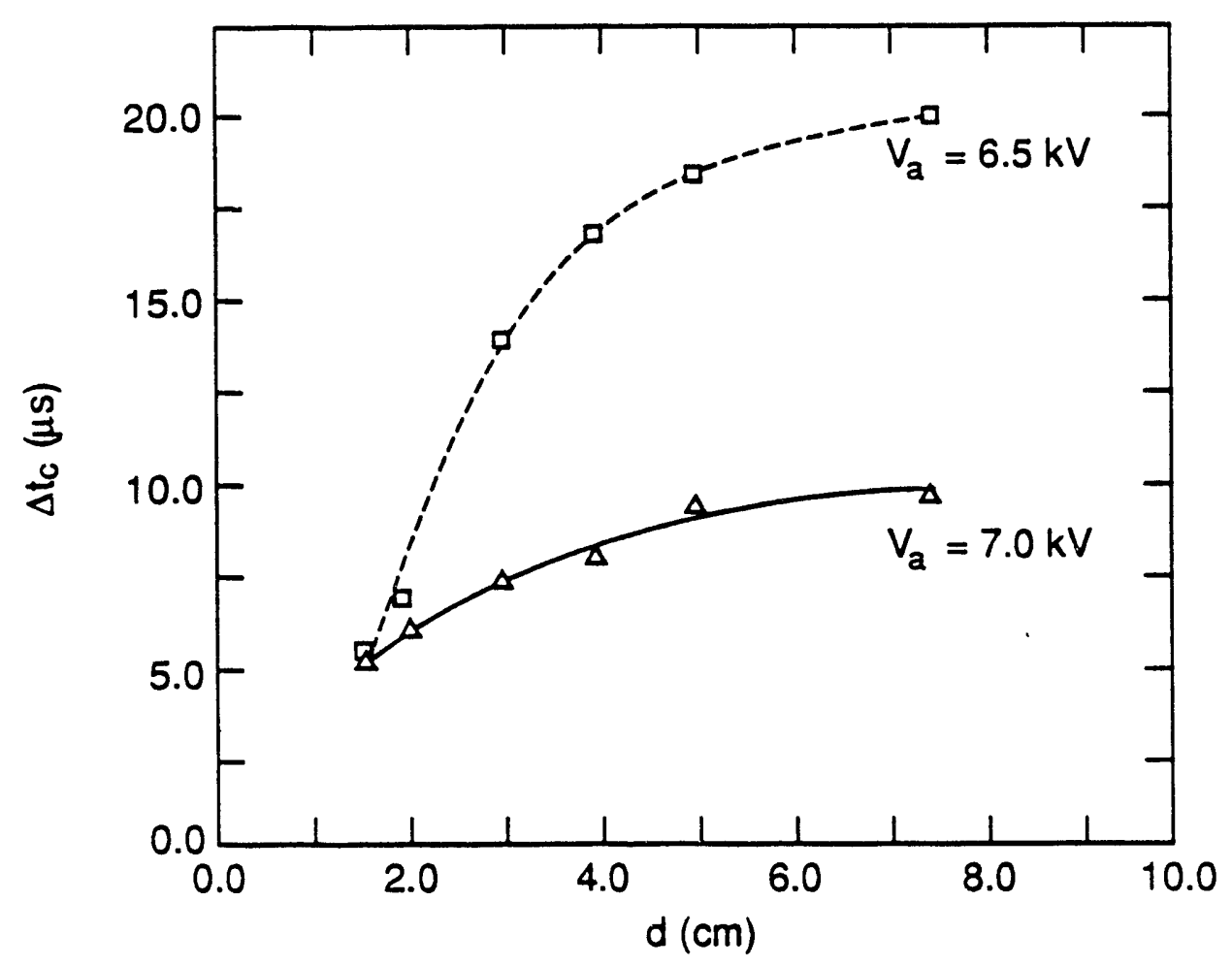

Figure 39. Minimum possible pulse time separation, $\Delta t_{c}$, versus gap spacing for the different indicated values of the applied voltage $V_{a}$ and for the case where there is no dielectric on the anode surface. 
known dependence of $q_{n}$ on $\Delta t_{n-1}$ as previously discussed [12]. The extent to which $q_{n}$ depends on $\Delta t_{n-1}$ is determined from measurements of $p_{1}\left(q_{n} \mid \Delta t_{n-1}\right)$.

Examples of measured conditional distributions $p_{1}\left(q_{n} \mid \Delta t_{n-1}\right)$ at different indicated values of $\Delta t_{n-1}$ and $d$ and for $V_{a}=10.1 \mathrm{kV}$ and $D=7.5 \mathrm{~cm}$ are shown in figure 40 together with the corresponding unconditional distribution $p_{0}\left(q_{n}\right)$ indicated by the dashed line. A strong positive dependence of $q_{n}$ on $\Delta t_{n-1}$ is seen from these data. This behavior is consistent with data shown in the previous section and can again be explained by the effect that moving negative-ion space-charge clouds from previous pulses have on the electric field at the cathode when the next discharge pulse develops.

The data on the unconditional distributions such as those shown in figures 35 and 36 can be used to compute the expectation values $\left\langle Q_{n}\right\rangle$ and $\left\langle 1 / \Delta t_{n}\right\rangle$ corresponding respectively to mean discharge-pulse magnitude and repetition rate. Examples of the results of such determinations for different values of $d, D$, and $V_{a}$ are shown in figure 41. Certain general trends are apparent from this figure. The mean discharge-pulse repetition rate generally decreases with decreasing $V_{a}$, and increasing $D$; whereas the mean discharge magnitude $\left\langle Q_{n}\right\rangle$, exhibits the opposite behavior, i.e., it decreases with increasing $V_{a}$ and decreasing $D$. Both $\left\langle 1 / \Delta t_{n}\right\rangle$ and $\left\langle Q_{n}\right\rangle$ may either increase or decrease with $d$ depending on the values of $D$ and $d$.

Figures 42 and 43 show plots of the expectation values $\left\langle q_{n}\left(\Delta t_{n-1}\right)\right\rangle$ versus $\Delta t_{n-1}$, where

$$
\left\langle q_{n}\left(\Delta t_{n-1}\right)\right\rangle=\int_{0}^{\infty} q_{n} p_{1}\left(q_{n} \mid \Delta t_{n-1}\right) d q_{n} .
$$

These results which were derived from equation(8) using data on the conditional distributions such as shown in figure 42 , indicate the strong dependence of $q_{n}$ on $\Delta t_{n-1}$ and are consistent with previous results [12] except at short distances $(d<0.6 \mathrm{~cm})$ at which a change in slope becomes evident as $\Delta t_{n-1}$ increases. This is seen most clearly by the data at $d=0.4 \mathrm{~cm}$ in figure 43 .

Several significant conclusions can be drawn from the results presented here. First, it is evident from the measured distributions $p_{0}\left(q_{n}\right), p_{0}\left(\Delta t_{n}\right)$, and $p_{1}\left(q_{n} \mid \Delta t_{n-1}\right)$ that, except for point-to-dielectric spacings very close to the critical value $d_{c}$ at which Trichel-pulse activity ceases, the presence of the dielectric surface has little or no effect in modifying the general stochastic behavior of the phenomenon. For sufficiently large spacings, the observed profiles of the unconditional pulse-amplitude and pulsetime separation distributions and the correlations between the amplitudes and time separations of successive pulses are consistent with those reported previously [12] for Trichel pulses that occur in air or $\mathrm{N}_{2}-\mathrm{O}_{2}$ mixtures when there is no dielectric on the planar electrode.

It can be argued from the data that give the dependencies of $\Delta t_{c}$ and $\left\langle 1 / \Delta t_{n}\right\rangle$ on $D$ shown in figures 37 and 41 , that the primary effect of the dielectric on Trichel-pulse development at any spacing $d$ arises from a reduction in the electric-field magnitude at the point electrode caused by the negative charge that accumulates on the dielectric 


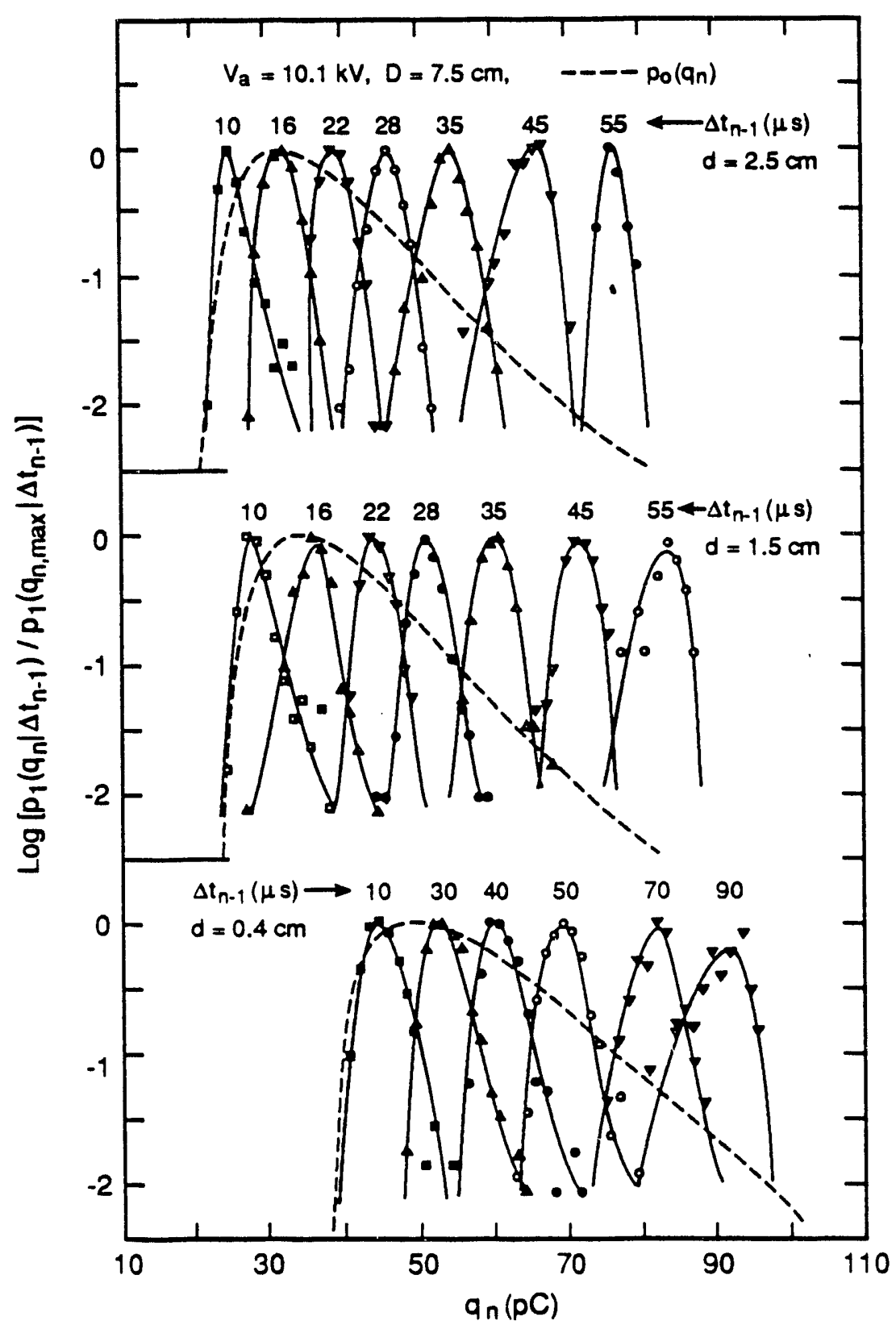

Figure 40. Measured conditional pulse amplitude distributions $p_{1}\left(q_{n} \mid \Delta t_{n_{1}}\right)$ at the indicated values for $\Delta t_{n-1}$ and gap spacing, and the corresponding unconditional pulse-amplitude distributions $p_{0}\left(q_{n}\right)$ shown by the dashed lines. The distribution have been normalized to the maximum values. 


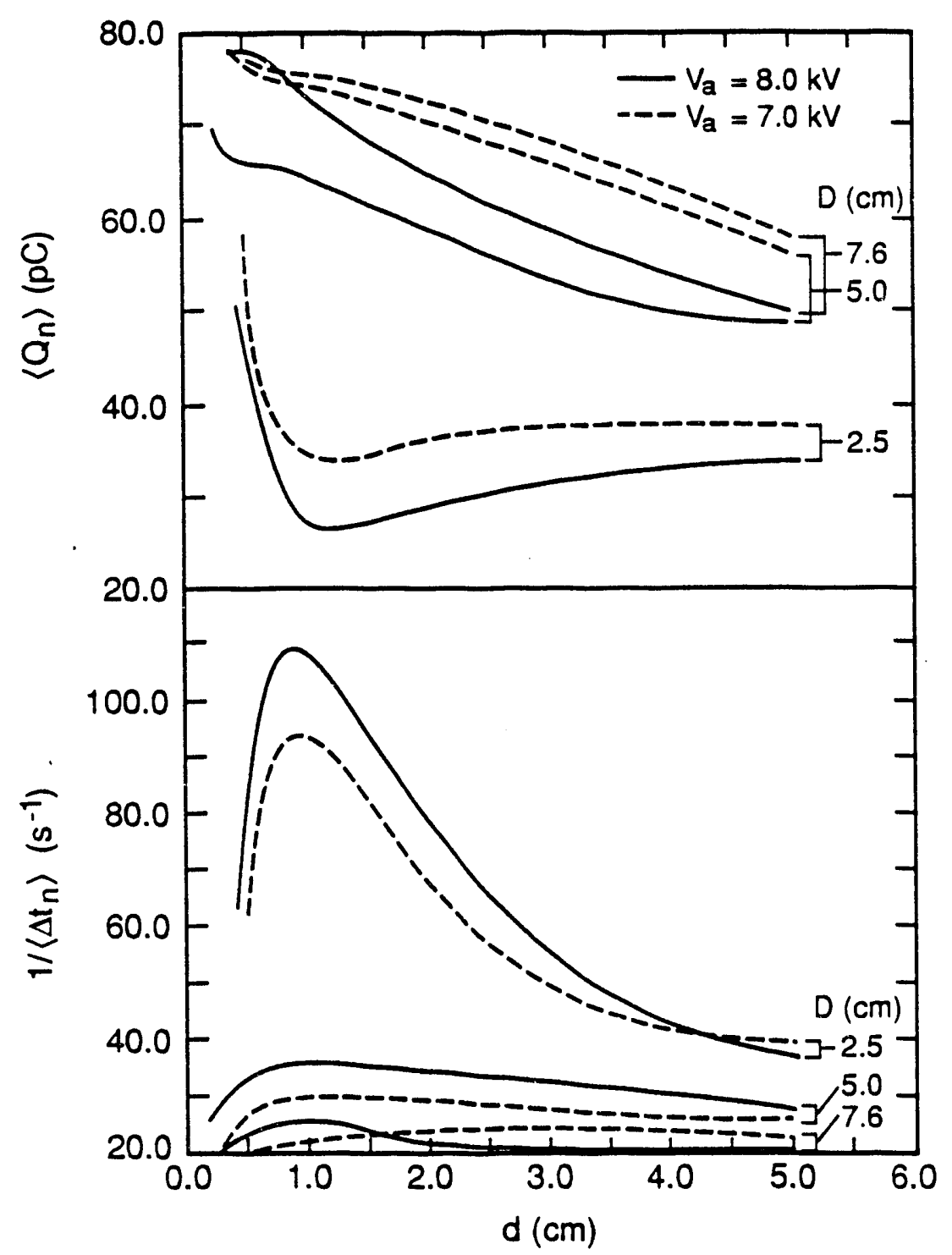

Figure 41. Dependencies of the mean pulse amplitude $\left\langle Q_{n}\right\rangle$ and repetition rate $1 /\left\langle\Delta t_{n}\right\rangle$ on gap spacing for the indicated values of $V_{a}$ and $D$ calculated from the distributions $p_{0}\left(q_{n}\right)$ and $p_{0}\left(\Delta t_{n}\right)$ such as shown in figures 35 and 36 . 


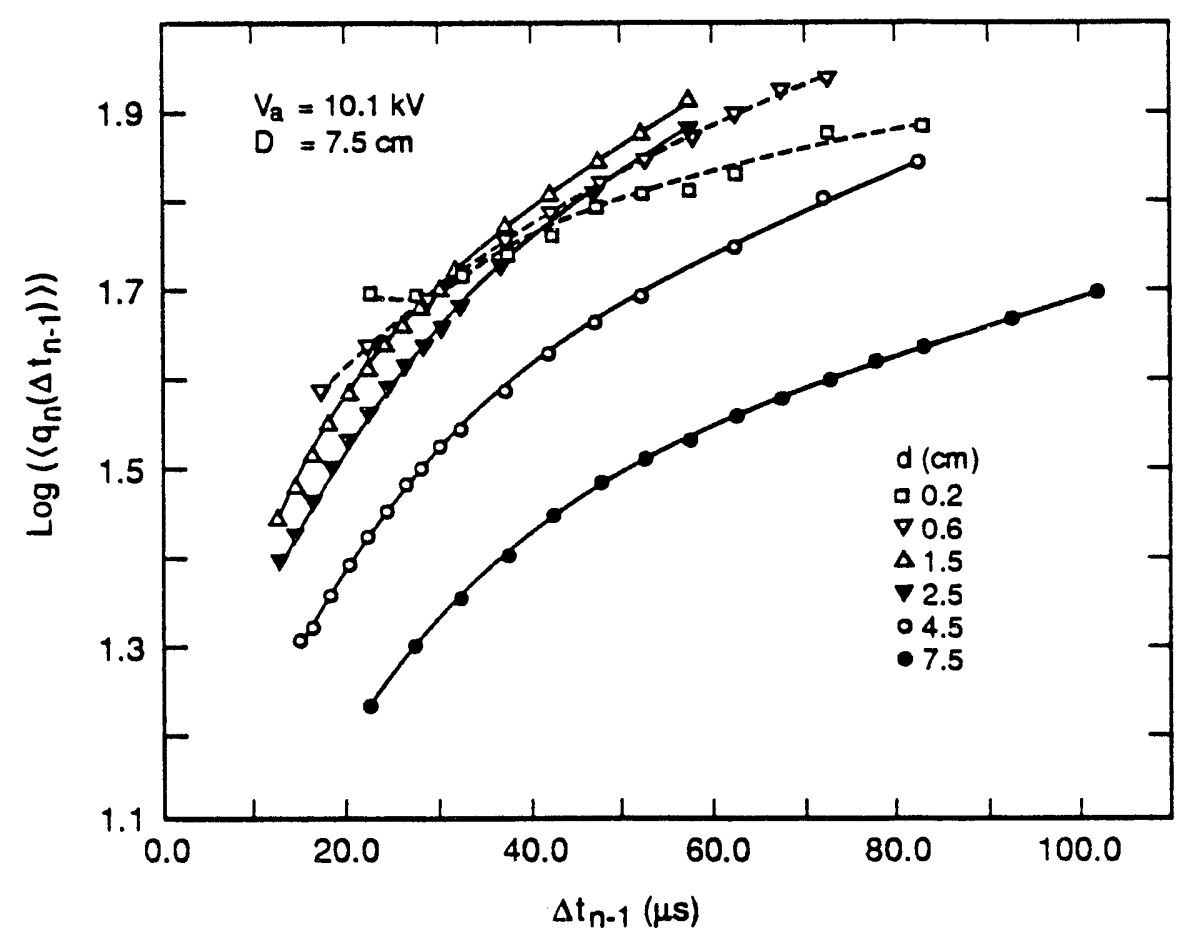

Figure 42. The expectation value $\left\langle q_{n}\left(\Delta t_{n-1}\right)\right\rangle$ versus $\Delta t_{n-1}$ at $V_{a}=10.1 \mathrm{kV}, D=7.5 \mathrm{~cm}$ for the indicated gap spacings.

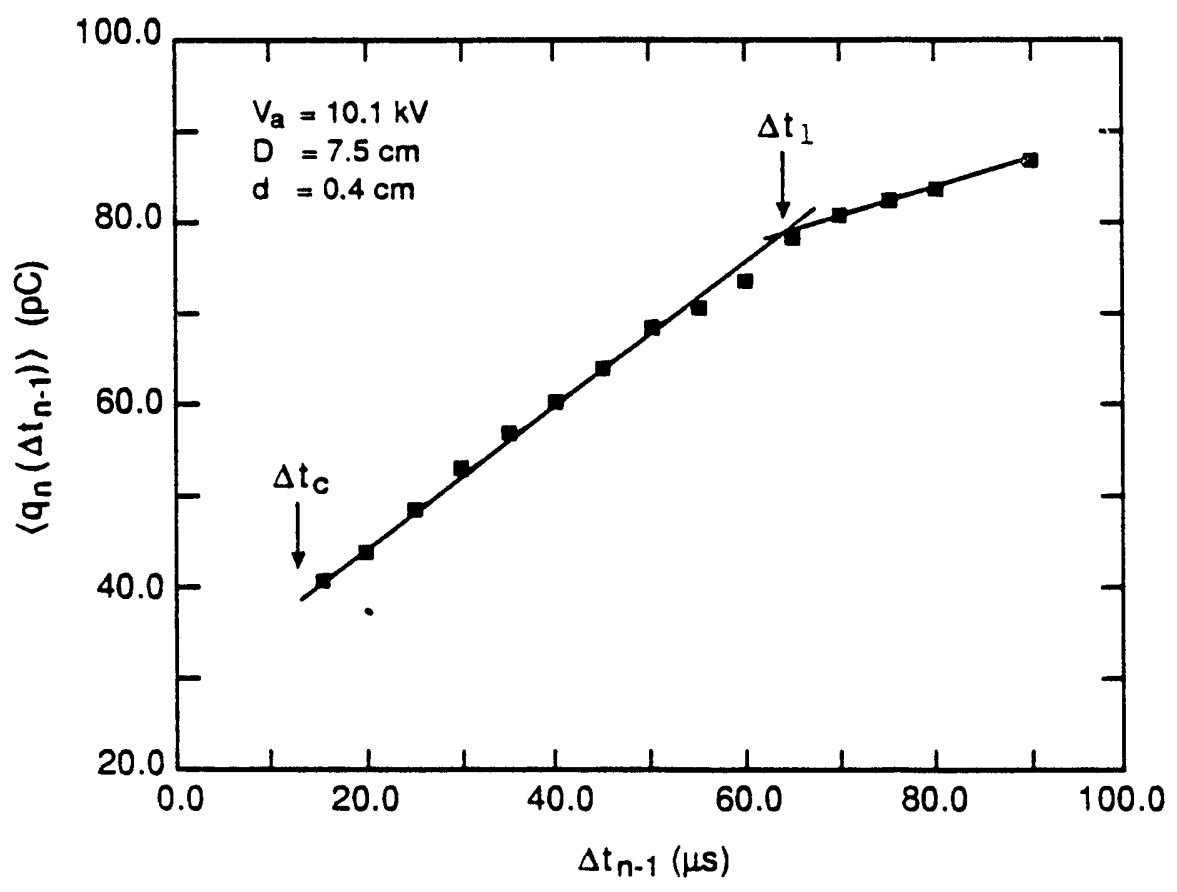

Figure 43. The expectation value $\left\langle q_{n}\left(\Delta t_{n-1}\right)\right\rangle$ versus $\Delta t_{n-1}$ for $V_{a}=10.1 \mathrm{kV}, D=7.5 \mathrm{~cm}$ and $d=0.4 \mathrm{~cm}$. 
surface during the discharge. This conclusion is supported by the $p_{0}\left(\Delta t_{n}\right)$ data in figures 35 and 38 for $d=2.0$ to $5.0 \mathrm{~cm}$ that were obtained respectively with and without a dielectric on the anode surface. The observed increases in both $\Delta t_{c}$ and the width of $p_{0}\left(\Delta t_{n}\right)$ which occur when the dielectric is introduced are consistent with a reduction of the electric-field magnitude equivalent to that which can be achieved by reducing the gap voltage. As previously noted, the parameters $\Delta t_{c},\left\langle 1 / \Delta t_{n}\right\rangle$, and the width of $p_{0}\left(\Delta t_{n}\right)$ are sensitive to small changes in electric-field magnitude at the point [12]. The effect of increasing the diameter of the dielectric surface is also similar to that of decreasing the gap voltage. The appearances of corresponding minima in $\Delta t_{c}$ and the widths of the $p_{0}\left(\Delta t_{n}\right)$ distributions at a particular gap spacing indicate that the field at the point electrode must attain a maximum at that spacing for a fixed $V_{a}$ when the dielectric is present.

It can be seen from figures 42 and 43 that the presence of the dielectric begins to influence the dependence of $q_{n}$ on $\Delta t_{n-1}$ significantly for small point-to-dielectric gap spacings close to the critical value $d_{c}$. From an examination of the data shown in figure 43 , it can be argued that for $\Delta t_{n-1}<\Delta t_{\ell}$, the dependence of $q_{n}$ on $\Delta t_{n-1}$ is due predominantly to the previously mentioned negative-ion space-charge effect which is a prominent characteristic in the stochastic behavior of Trichel pulses in the absence of the dielectric barrier [12]. The slope of the $\left\langle q_{n}\left(\Delta t_{n-1}\right)\right\rangle$ versus $\Delta t_{n-1}$ curve, however, is seen to decrease significantly at $\Delta t_{\ell}$. It is speculated that at $\Delta t_{n-1}=\Delta t_{\ell}$, the negative ions clear the gap and are presumably deposited on the dielectric. Thus, for $\Delta t_{n-1}>\Delta t_{\ell}$, the dependence of $q_{n}$ on $\Delta t_{n-1}$ could be governed primarily by the rate of charge dissipation or migration on the dielectric surface. It should be noted that the additional surface charge deposited by an earlier discharge pulse which is present when the next pulse initiates will act to reduce the field at the point electrode when this pulse develops and will thereby tend to inhibit its growth. Therefore, a dissipating residual surface charge from a previous pulse acts like the moving residual negative-ion space charge to cause $q_{n}$ to have a positive dependence on $\Delta t_{n-1}$.

Measurements performed with a vibrating plate type electric-field probe $[73,74]$ showed that after the corona discharge is turned off, the PTFE retains a quasipermanent negative surface charge that decays slowly with a time constant ( $>10^{4}$ seconds) which is much greater than the mean time between Trichel pulses. This observation suggests that if the dependence of $q_{n}$ on $\Delta t_{n-1}$, for $\Delta t_{n-1}<\Delta t_{\ell}$ in figure 43 is indeed due to an effect of surface-charge dissipation, it must correspond to a component of the charge density that is much more mobile than that detected with the field probe, i.e., it must decay with a time constant comparable in magnitude to the mean time between discharge pulses. This is consistent with recent observations of Jonscher and Owede [75] which indicate that the charge deposited on insulating surfaces can become increasingly more mobile as the charge density is increased.

The conclusions drawn here about the influence of a PTFE dielectric surface on Trichel-pulse discharge can be expected to apply in general to other types of insulating materials to the extent that these materials can acquire permanent or 
quasi-permanent charge from the corona. A preliminary survey made using other materials suggested that this is indeed the case.

\subsubsection{Limitations and Extensions of the SAPP Technique}

The system, as shown in figure 27, has certain limitations. First, it is designed to operate with dc voltages. Second, as discussed in ref. [60], it cannot give reliable measurements of certain conditional distributions if the time separations between events become shorter than either the TAC reset time or the MCA analog-to-digital conversion time. Third, it cannot be applied to nonstationary phenomena that drift on a time scale comparable to the time required to acquire statistically significant data. Fourth, it can only measure one distribution at any given time. Fifth, independent of the problems associated with timing in either the TAC or MCA, it does not employ broad-band detection methods and therefore cannot resolve pulses separated by times less than the width of the impulse response. Because of this limitation, it could not, for example, be applied to investigate P-D pulse bursts which as shown below occur in liquids and are known to occur in the positive corona of some highly electronegative gases like $\mathrm{SF}_{6}[55]$.

The restriction to dc volicages can be overcome by incorporating a phase marker such as a zero-crossing sensor to identify the phase associated with any given pulse time separation. The system has, in fact, been recently modified to allow measurement of conditional distributions restricted to specific phase intervals. For example it now allows measurement of the phase conditioned distributions, $p_{1}\left(q_{i}\left(\phi_{i}\right) \mid \Delta \phi_{j}\right)$ where $q_{i}$ is the amplitude of any P-D pulse that occurs with phase $\phi_{i}$ contained in the specified interval $\Delta \phi_{j}$. This type of distribution has been considered recently by others $[58,59]$. However, in addition to this distribution, our system is capable of measuring many others in which phase is specified. These include the distributions $p_{1}\left(q_{1}\left(\Delta \phi_{2}^{+}\right) \mid Q\left(\Delta \phi_{1}^{-}\right)\right)$and $p_{1}\left(q_{1}\left(\Delta \phi_{2}^{-}\right) \mid Q\left(\Delta \phi_{1}^{+}\right)\right)$, where $q_{1}$ is the amplitude of the first pulse to occur in either the positive or negative half cycle specified respectively by the phase intervals $\Delta \phi_{2}^{+}$or $\Delta \phi_{2}^{-}$and

$$
Q\left(\Delta \phi_{1}^{ \pm}\right) \doteq \Sigma_{j} q_{j}\left(\phi_{j}^{ \pm}\right) ; \phi_{j}^{ \pm} \in \Delta \phi_{1}^{ \pm}
$$

is the total charge associated with all P-D pulses occurring within phase interval $\Delta \phi_{1}^{ \pm}$of the preceding half cycle. Preliminary data obtained on these distributions for dielectric-barrier discharges at $60-\mathrm{Hz}$ voltages show that the charge deposited on a dielectric surface by partial discharges during one half cycle significantly affect the development of partial discharges during the subsequent half cycle.

Limitations on minimum time separations can be overcome in principle by using prerecorded data that can be obtained with fast-transient digitizers. However, if prerecorded data are to be used, it may be preferable to use computer software to 
determine the conditional distributions, rather than the hardware presented here. It should be emphasized that an important advantage of the method described here is that the conditional distributions are measured directly in real time. This is an advantage because it overcomes the problem of having to store enormous quantities of data required to determine conditional distributions by the software approach. For example, some of the second-order conditional distributions shown in figures 28 and 29 required about 10 minutes of data acquisition time in order to obtain acceptable statistics. This means that data on all but a very small fraction of the discharge events that occurred in this 10 minute period were discarded by the filtering procedure. Using the software approach, all the pulses that occurred during that time would have to be stored, which for the data shown means that more than $10^{9}$ pairs of numbers would have to be acquired just to determine one distribution. Also, because the present system works in real time, the distributions can be seen immediately as the data are accumulated.

If the P-D phenomenon is, or can become, nonstationary, then an independent method may be needed to determine the extent to which it is nonstationary. A periodic check of the unconditional pulse-amplitude and/or time-separation distribution will, in most cases, provide an indication of deviations from stationary behavior.

In the case of multiple P-D discharge sites, an ambiguity can occur in the interpretation of data from measurement of conditional distributions, particularly if the discharges from the different sites occur with nearly equal intensity. The measurement scheme discussed here works best if there is only one site at which P-D discharges are being generated with an intensity that is significantly above the background P.D level. The existence of pronounced correlations among successive P-D pulses would, in fact, indicate that the discharges are occurring at one, or a limited number of sites. The determination of this fact might be important in some diagnostic applications.

Finally, it should be pointed out that the present system could be modified to allow a more efficient use of the available data. One could, for example, use multiple MCA's in parallel to measure simultaneously more than one distribution. 


\title{
3 LIQUID DIELECTRICS RESEARCH
}

\author{
Task 03 \\ Kenneth L. Stricklett, H. Yamashita (Guest Scientist), \\ and Charles Fenimore \\ Electricity Division \\ National Institute of Standards and Technology
}

\subsection{Introduction}

Electric breakdown of liquid dielectrics is preceded by the growth of a streamer in the liquid. Recent studies of these phenomena, see for example Hebner [76] for a review, reveal that as a streamer propagates it follows a well defined pattern: Each stage or mode of growth may be distinguished by its structure, speed of propagation, and relative extent. Considerable effort has been devoted to describing the conditions that influence streamer propagation and initiation [77]. However, despite this activity, no clear consensus has been reached as to the mechanism for the initiation of the discharge. Proposals put forward have included: electron avalanche [78], cavitation [79], and electrostatic forces acting on the dielectric [80].

The mechanisms responsible for the initial growth of negative streamers appear to be distinct from those during its latter stages. Watson and Chadband [81] show initial growth of negative streamers to be consistent with the propagation of a cavity within the liquid. Similar results are reported for partial discharges (PD's), where photographic and optical studies [78, 82, 83] have established a clear correspondence between the growth of a cavity in the liquid and bursts of discrete current pulses emitted from a cathode. The results presented in this report were obtained during experimental investigations of the initiation and early growth of negative streamers in hexanes $[84,85]$. In section 3.2 , the initial growth of negative streamers generated by an impulse voltage is examined at pressures ranging from 0.1 to $1.1 \mathrm{MPa}$. The pressure dependence of the streamer initiation and inception voltages are shown. In section 3.3, partial discharges generated by the application of dc voltages are examined. A nonuniform field geometry is employed and the growth of cavities associated with partial discharges at a point cathode are photographed at high magnification. A simultaneous record of the discharge current is also obtained. This study employs an imagepreserving optical delay $[82,82]$, and the use of this device allows photography of the discharge at its inception. Such data allow detailed description of the temporal and spatial development of the cavity and provide a basis for the evaluation of models for the initiation of negative streamers. 


\subsection{Streamer Initiation in Hexanes}

For hydrocarbon-based dielectric liquids, four snodes of streamer growth are suggested $[76,87]$ for negative streamers; these are shown schematically in figure 44 . The 1st mode appears to be a single, relatively linear channel emanating from the cathode surface and connecting directly to a much more bushy structure, mode 2 . During modes 1 and 2, the streamer propagates at subsonic speeds. Although the 3rd mode originates at the 2nd mode, its structure differs from that of the 2nd mode, and its speed of propagation is significantly higher, approaching the sonic velocity. The transition from the 2nd to the 3rd mode is not continuous: streamer growth often stalls after the formation of the 2nd mode [88]. The 4th mode directly precedes breakdown and it appears to be a single streamer that propagates at supersonic speeds.

Experimental conditions influence streamer propagation; for example, the relative extent of the 2nd mode is sensitive to both the ambient pressure [89-91] and the purity of the fluid [92]. Indeed, it has been suggested that at sufficientiy high pressures, the 1st and 2nd modes may not exist at all [89,90,93]. Further systematic study of these effects is of value; and the present work was undertaken to examine the inception and early growth of negative streamers. For this purpose, highly magnified frame photographs of streamer growth at a needle tip as well as photographs of the full gap are obtained at pressures ranging from $0.1-1.1 \mathrm{MPa}$.

\subsubsection{Apparatus and Procedure}

Streamer growth is photographed using conventional shadowgraphic techniques and an image-converter camera $[87,89]$. The optical resolution is improved over that obtained in previous studies by mounting a microscope objective within the test cell. This configuration provides an optical resolution of approximately $1 \mu \mathrm{m}$ at $100 \times$ magnification.

The test gap consists of a steel gramophone needle and a stainless steel rod; the electrodes are enclosed within a brass cell and are immersed in the test fluid. The curvature of the needle is approximately parabolic, having a radius of curvature at its tip of approximately $40 \mu \mathrm{m}$. The rod electrode is $6.4 \mathrm{~mm}$ in diameter and has a hemispherical tip. The needle and rod are separated by a distance of $5 \mathrm{~mm}$.

A voltage impulse, having negative polarity, is applied directly to the needle and the rod electrode is connected to ground. The voltage waveform is approximately trapezoidal: the rise and fall times are $3 \mu \mathrm{s}(10-90 \%)$ and the voltage varies by less than $\pm 10 \%$ within a $4 \mu \mathrm{s}$ interval centered about the peak voltage. A precision resistive voltage divider and digital oscilloscope are used to record the waveform. The camera monitor pulse is also recorded and, together with the voltage waveform, these data determine the voltage at each frame within a photograph. Two measures 
of the effects of pressure are determined by these methods: the average streamer inception voltage, $\bar{V}_{\text {inc }}$, and initiation voltage, $V_{\text {init }}$. For purposes of this study, $V_{\text {init }}$ is the lowest voltage for which one streamer is observed in eight impulses, and is a measure of the minimum voltage required to produce a prebieakdown streamer. For voltages greater than or equal to the inception voltage, a second useful quantity may he deternined: the average voltage at the inception of the streamer $\bar{V}_{\text {inc. }}$ Values of $V_{\text {init }}$ and $\bar{V}_{\text {inc }}$ are measured using high magnification; furthermore, to avoid rapid deterioration of the needle tip and of the liquid, they are obtained without breakdown.

Ultra-pure grade hexanes are used both for this study and for the work discussed in section 3.3. The liquid is used as received without degassing or purification other than by filtration. While at atmospheric pressure, the liquid is continuously circulated and filtered through a $2 \mu \mathrm{m}$ pore size paper filter. The pressure within the cell is increased by compressing the liquid, which is accomplished by the use of small hydraulic cylinder connected to the cell. The application of pressure did not allow continuous filtration; however, pressure was released and the liquid circulated and filtered every 8-10 impulses.

\subsubsection{Results and Discussion}

\section{Photographic Observation of Streamer Behavior.}

Photographs of the full gap obtained at pressures of $0.1,0.7$, and $1.1 \mathrm{MPa}$ are shown in figure 45 . The effects of pressure are made evident in these photographs by a dramatic reduction in the extent of the 2 nd mode and by changes in tine structure of the 3rd mode: it may be noted that the latter is less dense and has fewer branches at high pressure. The 1st and 2nd modes may be readily identified in the photographs obtained at atmospheric pressure and remain visible up to $0.7 \mathrm{MPa}$, however they are not resolved in the low-magnification photographs for pressures of $0.9 \mathrm{MPa}$ and above.

For comparison, high magnification photographs obtained at pressures of $0.1,0.5$, and $1.1 \mathrm{MPa}$ are shown in figure 46. In contrast to the photos taken at low magnification, the characteristic stem and bush structure is clearly evident at all pressures. Furthermore, the images are sufficiently well-resolved to indicate the dynamical behavior of the streamer: upon close examination of these photographs, it is evident that the 1st mode expands and contracts repeatedly as the streamer propagates. It was further noted, that the frequency and amplitude of oscillation of the 1st mode increase with pressure. Indeed, at the highest pressure examined, the 1st mode completely disappears and reappears. The maximum diameter and length of the 1st cathode mode are, however, relatively insensitive to pressure and are on the order $4 \mu \mathrm{m}$ and $8 \mu \mathrm{m}$ respectively. The $2 \mathrm{nd}$ mode did not exhibit such dramatic oscillations. 
The oscillations in the diameter of the 1st cathode mode may be consistent with intermittent light emission and charge injection observed during the propagation of negative streamers $[94,95]$. Studies of partial discharge phenomena at a point cathode in the same material [85] show current to be highly correlated with the presence of a bubble-like structure at the electrode. It seems likely that significant charge injection can only occur when the streamer is in contact with the electrode; and thus that the prebreakdown streamer provides a preferred path for current flow. Indeed, studies of full breakdown show the conducting channel to lie along the path of a prebreakdown streamer.

\section{Streamer Initiation and Inception Voltages.}

The methods described allow the effects of changes in the ambient pressure and in the peak voltage on the streamer inception voltage to be examined. Values of $\bar{V}_{\text {inc }}$ are estimated from eight trial pulses and are measured using a $217 \mathrm{~ns}$ frame interval. The results of these measurements for pressures of $0.1,0.5$, and $0.9 \mathrm{MPa}$ are shown in figure 47 , where $\bar{V}_{\text {inc }}$ are plotted on representative traces of the voltage waveform. Clear trends in both the formation time and inception voltage are apparent. At $0.1 \mathrm{MPa}, \overline{\mathrm{V}}_{\text {inc }}$ has a strong dependence on the voltage waveform: the time required for streamer inception decreases and the voltage at inception increases with applied voltage. Although the formation time decreases with faster rising impulses, it may be noted that the inception voltage is relatively constant for the data obtained at $0.9 \mathrm{MPa}$. Indeed $\overline{\mathrm{V}}_{\text {inc }}$ appears to approach a value that is relatively independent of pressure as the peak voltage is increased. These observations lend support to the results of Kao and McMath [96], who show for both transformer oil and n-hexane that increased pressure has little or no influence on breakdown voltage for fast rising voltages. In their study, the effect of increased pressure was found to be negligible when the rate of rise of the applied voltage exceeded $4 \mathrm{MV} / \mathrm{cm} \mu \mathrm{s}$. For the results presented here, the rate of rise increases with applied voltage. Numerical methods [97] used to estimate the electric field at the needle tip yield $5.5 \times 10^{6} \mathrm{~V} / \mathrm{cm}$ at a $30 \mathrm{kV}$ applied potential. This value together with the greatest rate of voltage rise shown in the figure yield $6.6 \mathrm{MV} / \mathrm{cm} \mu \mathrm{s}$ which compares favorably with the value given by Kao and McMath.

The effect of pressure on the streamer initiation voltage is shown in figure 48 . $V_{\text {init }}$ is seen to inciease with pressure from 0.1 to $0.5 \mathrm{MPa}$ and to be relatively independent of pressure above $0.5 \mathrm{MPa}$. Peak voltages of -33 and $-46 \mathrm{kV}$ were used at 0.1 and $0.3 \mathrm{MPa}$, respectively, and $-53 \mathrm{kV}$ for pressures of $0.5 \mathrm{MPa}$ and above. In each case, streamer initiation occurred near the peak voltage. 


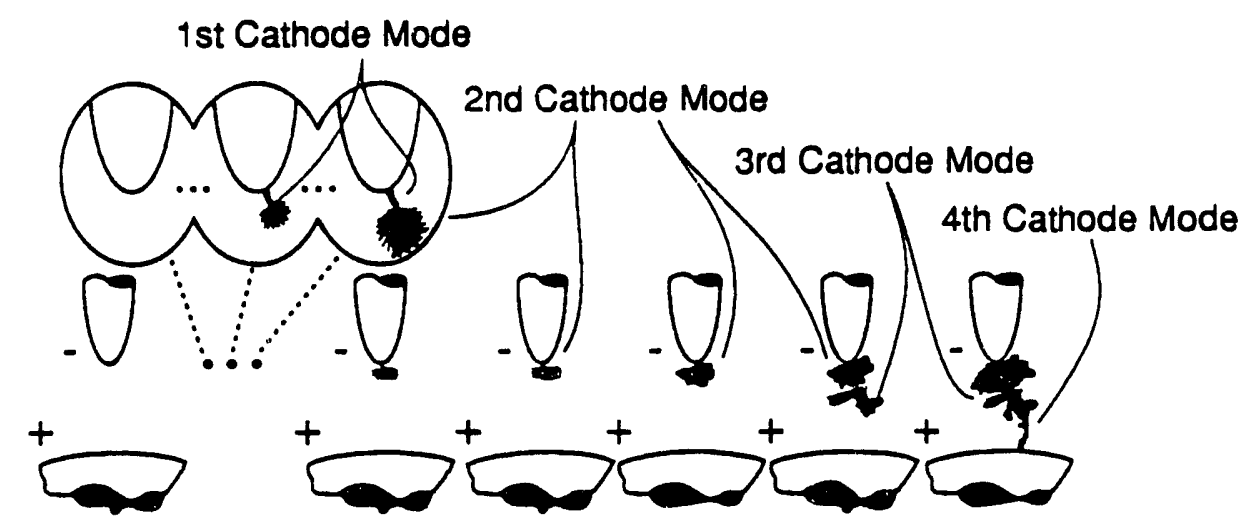

Figure 44. Cathode streamer development. The four modes of streamer growth are shown, (Hebner [76]). 


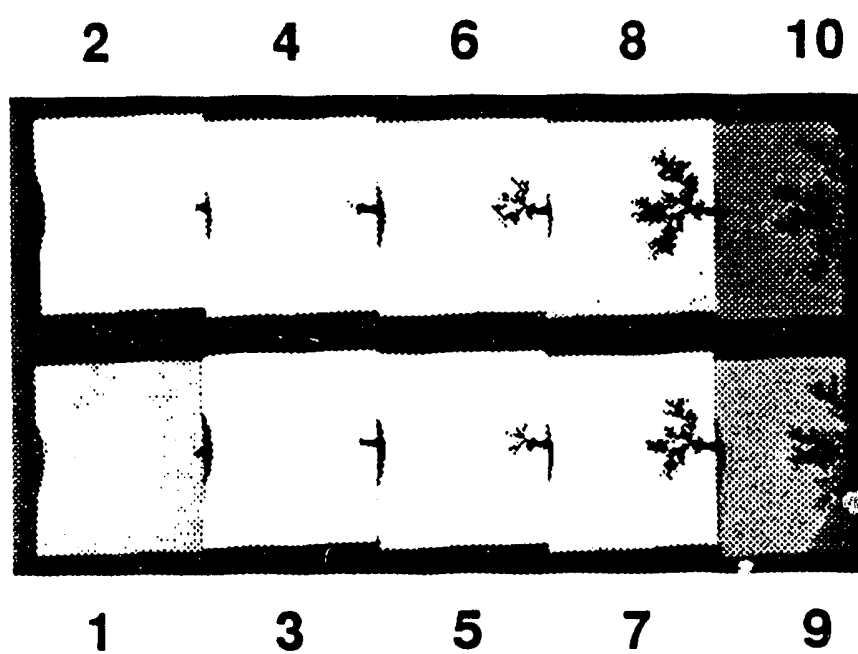

\section{(a) $0.1 \mathrm{MPa}$}

\section{$5 \mathrm{~mm}$}

(b) $0.7 \mathrm{MPa}$

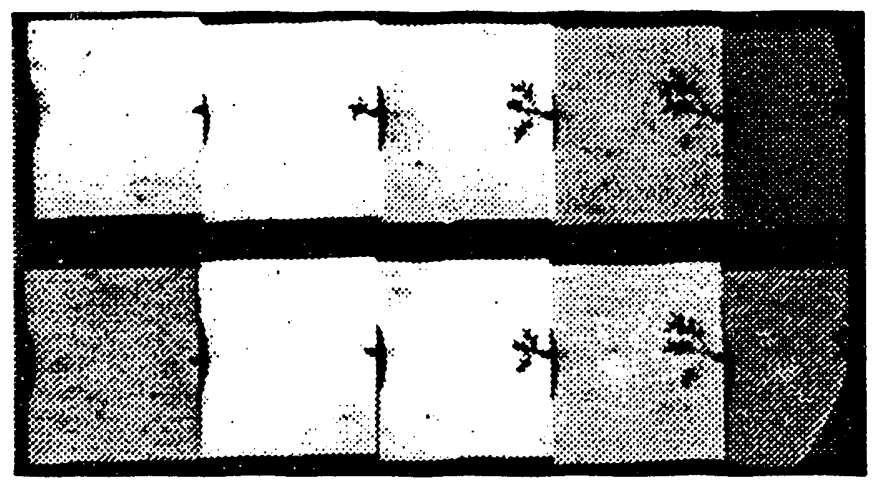

(c) $1.1 \mathrm{MPa}$

Figure 45. Low magnification photographs of prebreakdown streamers. The needle electrode is shown on the right in each of these photographs. Modes 1 and 2 may be readily identified in the photographs obtained at $0.1 \mathrm{MPa}$. The frame interval is $512 \mathrm{~ns}$ and the frame sequence is shown in (a). An impulse having a peak voltage of $-93 \mathrm{kV}$ is applied to the needle. 


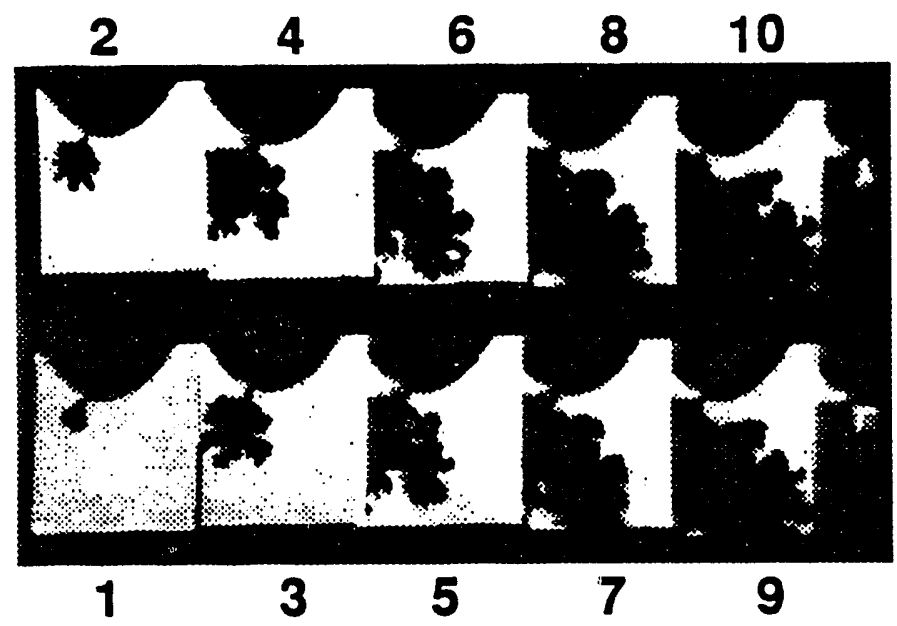

(a) $0.1 \mathrm{MPa}$

$V_{p}=-46 k V$ $50 \mu \mathrm{m}$

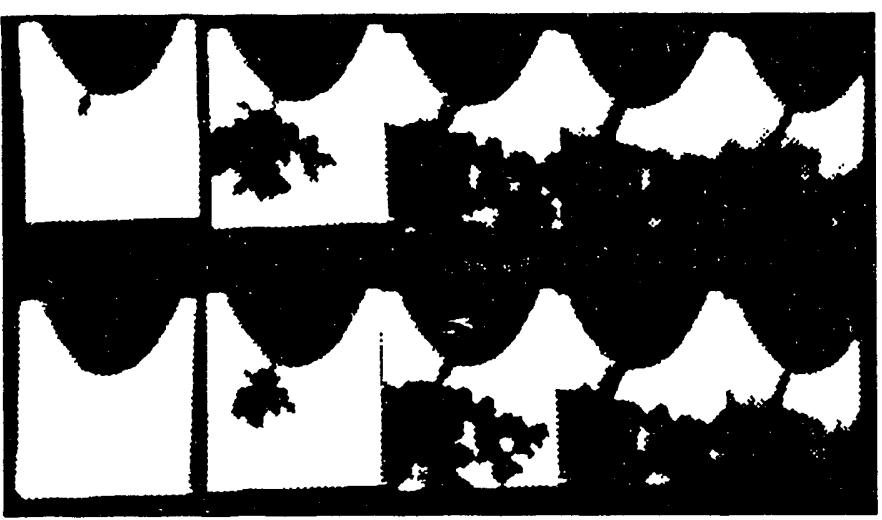

(b) $0.5 \mathrm{MPa}$

$V_{p}=-81 k V$

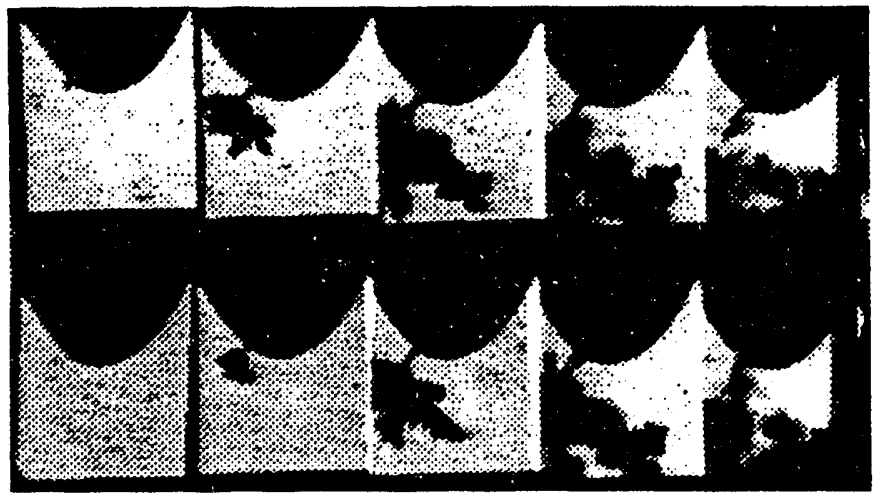

(c) $1.1 \mathrm{MPa}$

$V_{p}=-67 \mathrm{kV}$

Figure 46. High-magnification photographs of prebreakdown streamers. The pressure and peak voltages are indicated beside each photograph and the frame interval is $217 \mathrm{~ns}$. 


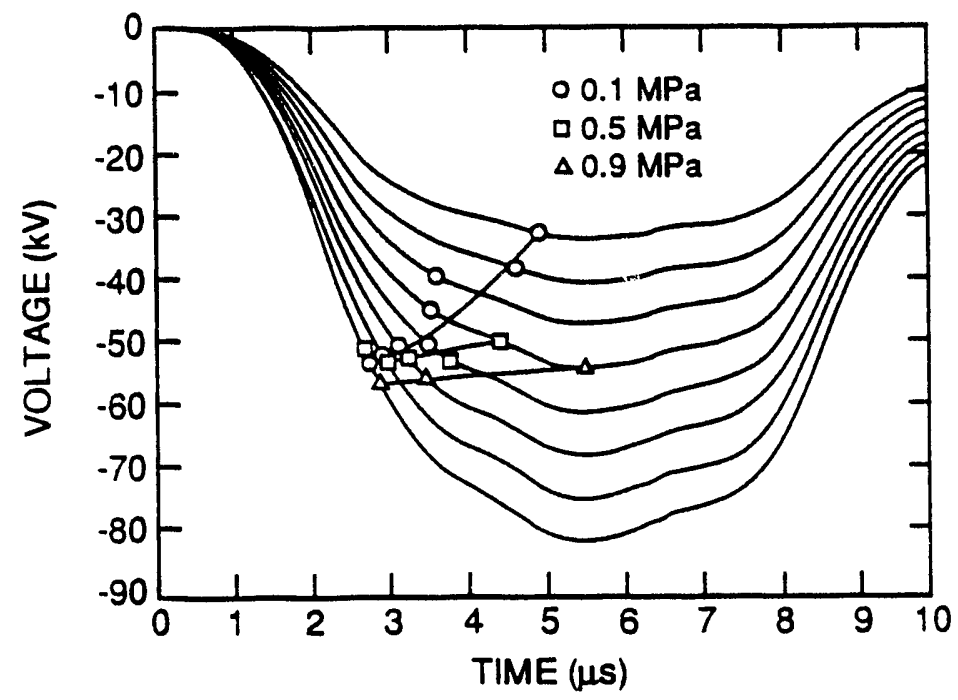

Figure 47. Pressure and waveform effects on streamer inception. The average streamer inception voltages $\bar{V}_{\text {inc }}$, are plotted on representative traces of the voltage waveform. Values obtained at pressures of $\bigcirc, 0.1 ; \triangle, 0.5$; and $\square, 0.9 \mathrm{MPa}$ are shown.

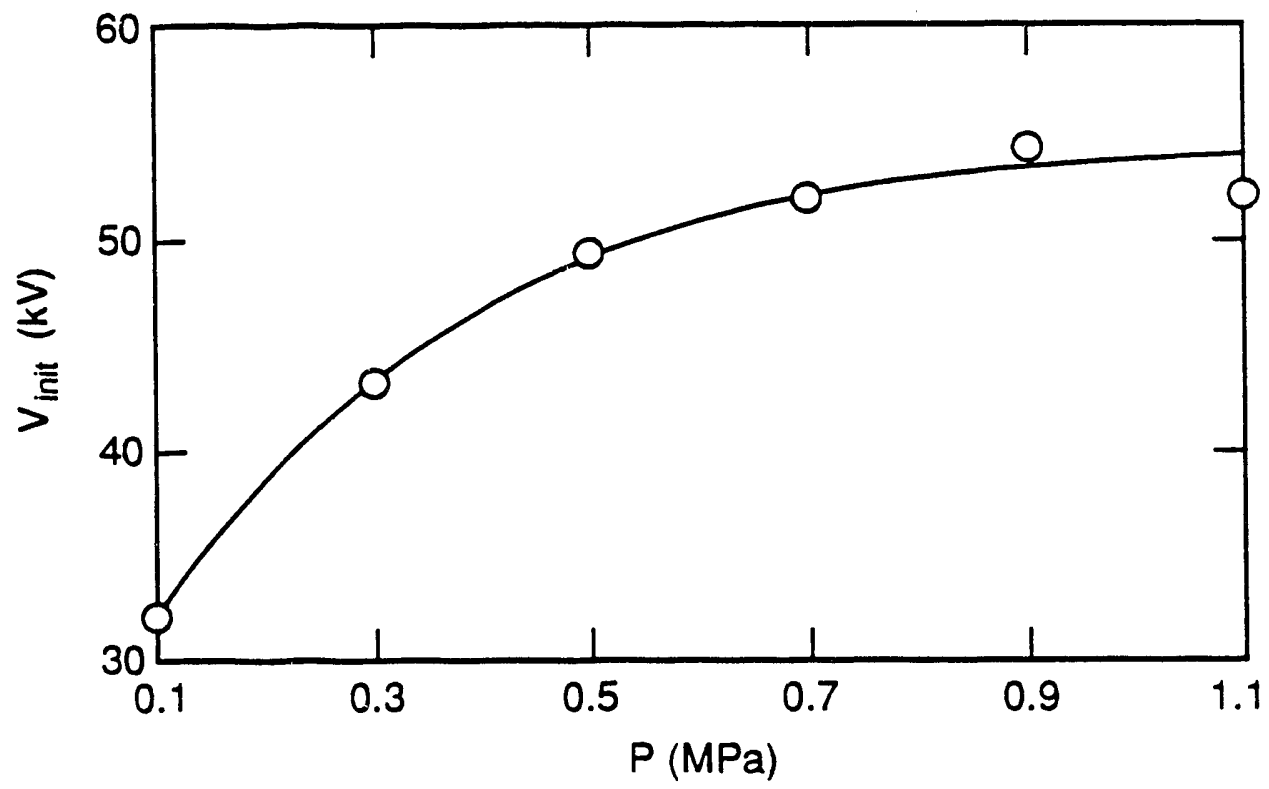

Figure 48. Pressure dependence of the streamer initiation voltage, $V_{\text {init }}$. 

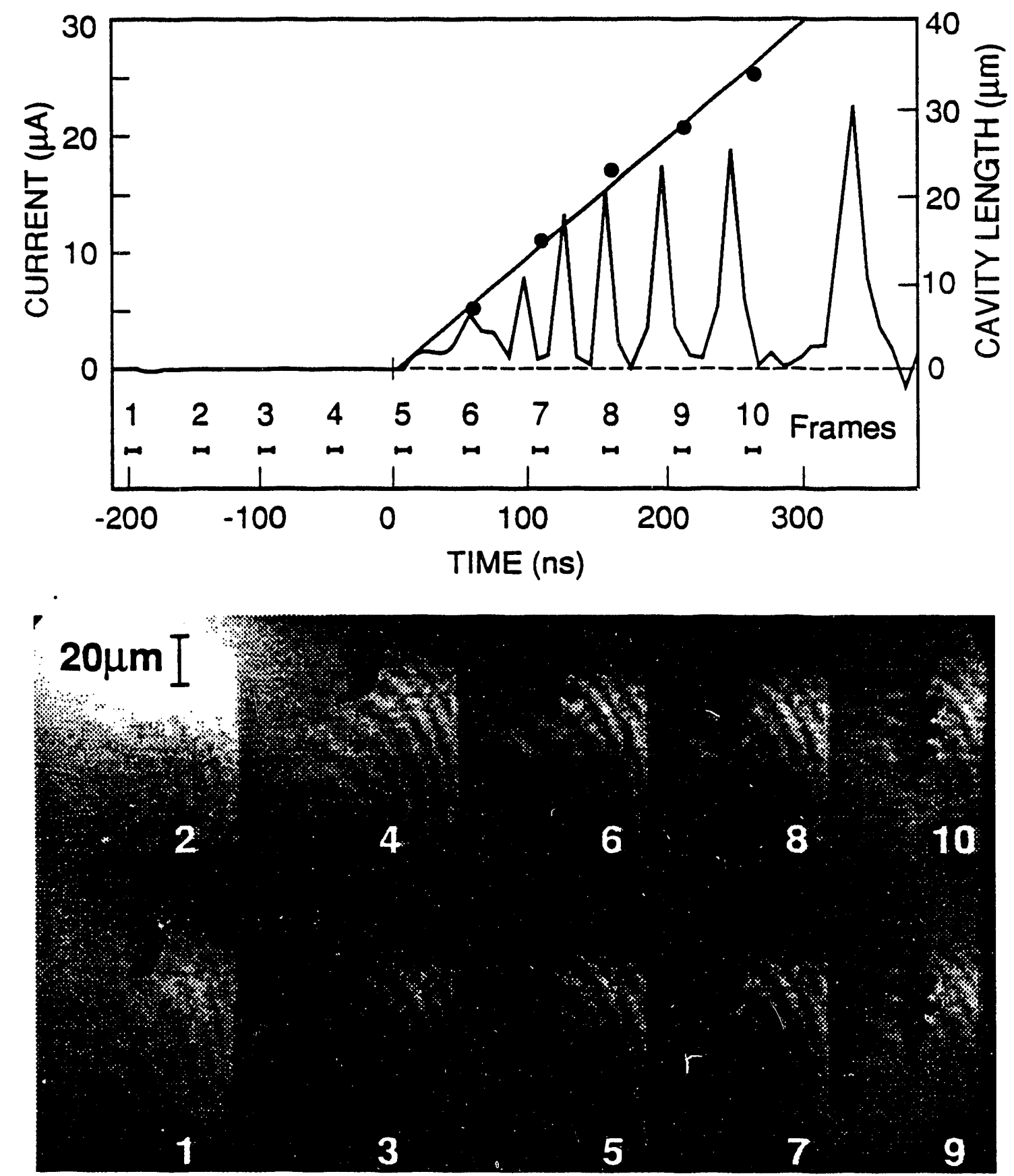

Figure 49. Partial discharge record obtained at $17.5 \mathrm{kV}$. The frame interval and exposure are 51 and $10 \mathrm{~ns}$ respectively. The current waveform and the extent of the cavity are plotted in the upper panel. 


\subsection{Voltage Conditions}

For dc voltages near the threshold for P-D activity, P-D's occur infrequently and are separated by time intervals that are widely distributed. Furthermore, significant current may only persist for times of the order of microseconds. These characteristics present a technical challenge: photographing a short lived event that may occur essentially at random. The methods employed allow two independent records of the P-D to be obtained simultaneously: measurement of P-D current and frame photography of the cavity.

\subsubsection{Experimental Apparatus}

The test gap consists of a steel needle and stainless steel rod separated by $3.2 \mathrm{~mm}$. The electrodes are enclosed within a brass cell and are immersed in the test liquid. The liquid is used as received without degassing or further purification. The rod electrode is $6.4 \mathrm{~mm}$ in diameter and has a hemispherical tip, and is connected to a high-voltage dc power supply. The needle electrode is $1.0 \mathrm{~mm}$ in diameter and has a conical point with an apex angle of $30^{\circ}$ and a tip radius of $1 \mu \mathrm{m}$.

A continuous-wave argon laser is used to illuminate the needle tip, and frame photographs of the discharge are obtained by shadowgraphic methods and the use of an image-converter camera. The total magnification is $200 \times$ and the optical resolution is sufficient to fully resolve objects less than $2 \mu \mathrm{m}$ in diameter. The use of an image-preserving optical delay allows the framing sequence to begin much closer to, and for some frame intervals before, the on set of the partial discharge.

A broadband transimpedance amplifier $[98,99]$ is used to measure the P-D current. The amplifier is designed to provided a low-noise background against which lowlevel P-D currents may be detected. The amplifier has three stages: a low-noise, broad-bandwidth, current-sensitive preamplifier and low-gain and high-gain buffer amplifiers. The combined system bandwidth is estimated to be $35 \mathrm{MHz}$ and the equivalent noise is $30 \mathrm{nA}(\mathrm{rms})$. The charge sensitivity of the system is estimated to be less than $0.7 \mathrm{fC}$. The amplifier is connected directly to the needle electrode and the current waveform is recorded digitally. A digital oscilloscope having a sample rate of $10^{8} \mathrm{samples} / \mathrm{s}$ is used to obtain the results shown here. Both the digital oscilloscope and the framing camera derive their trigger signals from the P-D current.

\subsubsection{Partial Discharge Records}

An example of a P-D record, obtained at an applied potential of $17.5 \mathrm{kV}$, is shown in figure 49. Note that the first few camera frames precede the initiation of the current waveform. The data at hand allow an estimate of the time interval between the 
initial current pulse and the onset of cavity growth. As shown, the current waveform begins $51 \pm 5 \mathrm{~ns}$ before the midpoint of the sixth frame, the first frame in which clear evidence of the cavity appears. The estimated uncertainty in the position of frame six is $\pm 8 \mathrm{~ns}$; combining these uncertainties in quadrature yields $51 \pm 9 \mathrm{~ns}$ and thus provides an upper bound of $60 \mathrm{~ns}$. The extent of the cavity in the sixth frame, however, suggests that the time interval is likely to be significantly less. In the upper panel, the length of the cavity is plotted against the frame number and fit to a linear function. Such a fit provides an estimate for the origin of the cavity that is nearly coincident with the initiation of the current waveform. However, in the absence of a more accurate determination of the instantaneous rate of expansion of the cavity at its inception, we are reluctant to apply this correction and prefer to report the upper bound.

The correlation between the onset for cavitation and PD current noted here lends support to recent experimental results obtained in cyclohexane [78]. Results obtained in nitrobenzene suggest that significant charge may be injected into the liquid prior to streamer initiation $[100,101]$, and that the onset for charge injection precedes that for cavitation by as much as $4 \mu \mathrm{s}$ [100]. The presence of charge in the fluid would result in a force on the fluid of the proper sign to produce the observed cavitation.

The linear fit also provides an estimate of the average rate of expansion of the cavity. Furthermore, since the rate of expansion depends on the forces acting on the liquid, an estimate of the effective pressure within the cavity, $P_{\mathrm{in}}$, may be obtained. Assuming inviscid incompressible flow, and approximating the cavity by an expanding sphere of radius $r$, the instantaneous work done in expanding the cavity is

$$
P_{\text {in }} d v=P_{\text {amb }} d v+\frac{2 S}{r} d v+\frac{3}{2} \rho \dot{r}^{2} d v .
$$

The terms include: the work done against ambient pressure, $P_{\text {amb }}$, and surface tension, $S$; and the kinetic energy imparted to the surrounding liquid. The average rate of expansion of the cavity, $\dot{r}$, is approximately $20 \mathrm{~m} / \mathrm{s}$ and the density, $\rho$, and surface tension of hexanes are approximately $0.66 \mathrm{~g} / \mathrm{cm}^{3}$ and $1.8 \times 10^{-2} \mathrm{~N} / \mathrm{m}$ respectively. For the conditions described, the kinetic energy is the largest term and $P_{\text {in }}$ is approximately $0.4 \mathrm{MPa}$.

The required pressure may be provided by local heating of the liquid, as may occur during electron avalanche, and by electrostatic forces. The form of the cavity, however, suggests that the forces acting on the liquid are highly directional, and thus that the gas dynamic pressure within the cavity is of limited importance. We believe these observations lend support to the results reported by Watson and Chadband [81] and further suggest that, from its inception, the growth of the cavity may be described by the electrostatic forces acting on the liquid.

The current waveform is relevant to describing the conditions at the initiation of the P-D. In over $90 \%$ of the current waveforms, clear evidence of a continuous current 
is detected at the initiation of the waveform. Furthermore, whereas the later current pulses tend to grow monotonically in amplitude, the first two pulses are frequently of comparable amplitude. Indeed, P-D's are observed that consist of only a doublepulse structure. These observations suggest that the mechanisms producing the measured current at the origin of the cavity growth differ from those at later times. Two mechanisms that give rise to continuous currents are suggested: the motion of charge trapped in the liquid or at the interface between the liquid and the metal electrode would produce a current in the external circuit [102], also the reduction in the capacitance of the cell due to a reduction in the dielectric constant of the material near the needle tip would produce a continuous current. 


\subsection{Conclusions}

The effects of increased ambient pressure on the initiation and growth of negative streamers in hexanes are observed using a $100 \times$ high-magnification optical system with $1 \mu \mathrm{m}$ resolution. The existence of the 1 st cathode streamer mode is clearly shown at pressures ranging from 0.1 to $1.1 \mathrm{MPa}$. The 1st cathode mode is approximately $4 \mu \mathrm{m}$ in diameter and $8 \mu \mathrm{m}$ in length at the transition to the 2nd mode. Dynamical behavior of the 1st cathode mode is shown; the diameter of the stem appears to oscillate as the streamer grows and, at high pressure, the stem completely disappears and reappears although the 2 nd mode continues to exist. The behavior of the 1st cathode mode may be associated with intermittent charge injection and the stepped propagation of negative streamers noted in previous studies. The 3rd mode becomes thinner and less branched at higher pressure. The streamer-initiation voltage increases with pressure, but the effect of pressure on the streamer-inception voltage decreases at higher applied voltage.

Highly resolved photographs of the initiation of P-D's in liquid hexanes are obtained for dc voltages. The cavity growth at a point cathode is nonisotropic, which suggests that electrostatic forces are of primary importance in driving its expansion. The onset for P-D current precedes or is simultaneous with the initial growth of the cavity. An upper bound of $60 \mathrm{~ns}$ between the first current pulse and the growth of the cavity is estimated. 


\title{
4 FAST TRANSIENT MEASUREMENTS
}

\author{
Task 04 \\ G.J. FitzPatrick and Y.X. Zhang (Guest Scientist) \\ Electricity Division \\ National Institute of Standards and Technology
}

\subsection{Introduction}

The purpose of an impulse voltage measuring system is to provide a means of reducing the high voltage signal to levels which are compatible with data recording equipment and this requires that the scale factor of the system be constant throughout the frequency range of interest. Most impulse measurement systems used by industrial laboratories invariably introduce some distortion of the input signal due frimarily to inadequate bandwidth, voltage coefficient, and some other factors. This distortion may be either negligible or totally unacceptable, depending on the allowable error associated with the particular measurement requirement. According to IEC and IEEE standards [103,104], a system which is used to measure standard lightning impulses should have errors of less than $3 \%$ and $10 \%$ for peak voltage and front time, respectively. The standards also recommend that certain aspects of the dynamic behavior of the measurement system should be evaluated by using parameters obtained from the step response. There is, however, no reliable relationship between the errors of a measurement system and its step response parameters. In fact, it has been shown that some measurement systems exhibit step response parameters that fall within the limits given by IEC and IEEE but that actually produce unacceptably large errors in the measurement of high voltage impulses $[105,106]$.

A more reliable and simplified method has been recommended in the new draft of IEC- 60 that is based on simultaneous measurements of a high voltage impulse by an independent reference system and the system under test [107]. As a first step, international comparative measurements were made in four National Laboratories and the relative differences among them were reported [109]. Investigations have also been made of the interactions between two systems configured for simultaneous measurements, and of methods for minimizing these interactions [106].

The comparative method establishes the uncertainty in measurements of a particular type of impulse for a measurement system by comparison against a reference measurement system having overall uncertainties for peak voltage of less than $1 \%$ over its range of use [107]. The key to the comparative measurement technique is that the reference system has to be built and carefully characterized. Without a stable and precise reference systern, it is usually impossible to use the comparative method to estimate the uncertainty of another system. There are, however, techniques that 
utilize a pair of complementary measurement systems to detect measurement errors in each system, but the technique requires that the measurement systems have different error sources [108].

As a part of a series of investigations into high voltage impulse measurements, a resistive impulse measurement system was specially designed, built, and calibrated at NIST. It is anticipated that this system along with some Kerr electro-optical devices will be used as the NIST reference systems. This report documents the design, construction, and characterization of this fast impulse voltage measurement system, which is rated for peak voltages up to $300 \mathrm{kV}$.

\subsection{Resistive Voltage Divider Design}

\subsubsection{General Considerations}

The resistive voltage divider, designated as NIST4, serves as the primary component of the reference measurement system. It was designed to accurately measure full lightning impulses up to $300 \mathrm{kV}$. The voltage range was selected for several reasons. First, it was similar to devices used as reference systems in the national laboratories of other countries so that direct comparison between the devices would be reasonable. Second, compared with a divider rated for megavolt impulses, construction was simplified because of its smaller physical size. Additionally, the proposed draft, IEC 60-2, permits the qualification of a larger divider by the comparative measurement technique with a smaller reference divider at $20 \%$ of the rated voltage of the larger one [107]. A reference divider rated at $300 \mathrm{kV}$ can therefore be used to compare with dividers having ratings of up to $1500 \mathrm{kV}$ [107]. The NIST4 divider has a nominal resistance of $10 \mathrm{k} \Omega$, which is typical for most resistive dividers. A divider ratio of 1000:1 was selected rather than a larger one to give a signal-to-noise ratio that was high enough that degradation of the divider output signal by pickup of radiated noise would be minimized. Both digital recorders and digital storage oscilloscopes require lower signal levels than this ratio provides at voltages exceeding $200 \mathrm{kV}$ and further attenuation is needed. Attenuators are used at the recorder end of the signal cables. The divider and attenuators must have adequate bandwidth for the frequency range of interest. A resistive divider must have sufficiently fast transient response to precisely measure steep impulse voltages such as the $1.2 \mu$ s front time standard lightning impulse. It must have a stable divider ratio for the voltage, temperature and humidity range of use. The voltage coefficient and environmental effects must be negligible. Mechanical effects that unfortunately have been ignored in many cases are also important. The divider ratio must not be affected by these factors as well. 


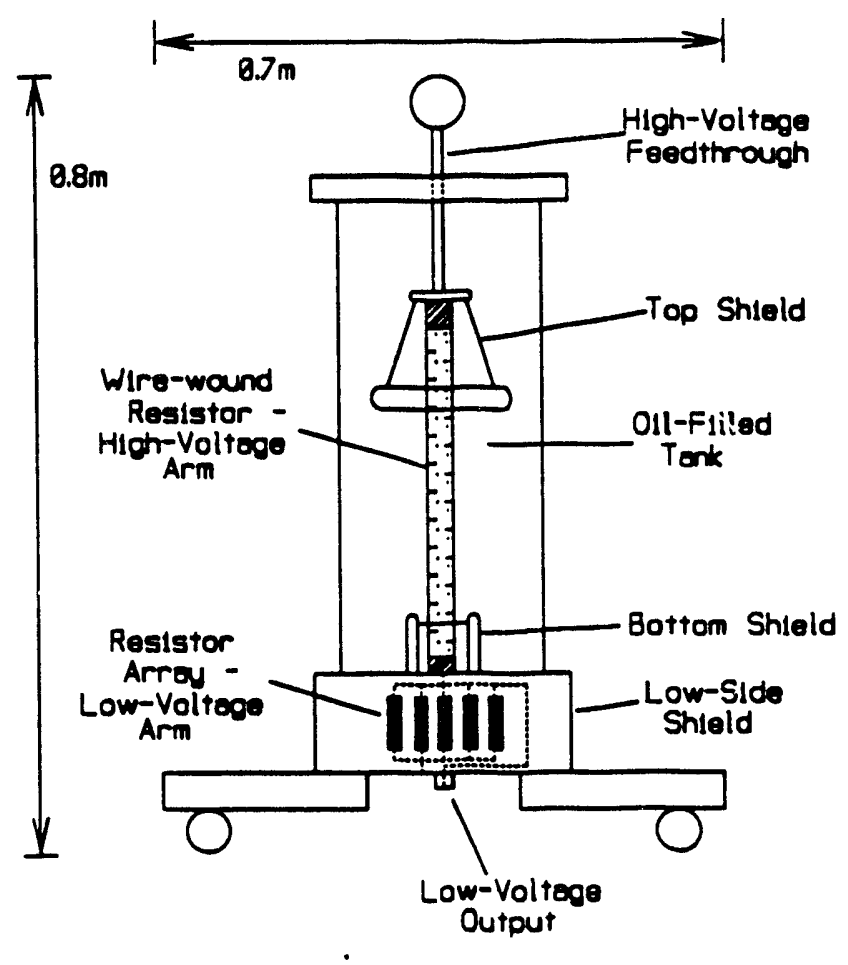

Figure 50. Desigu of NIST4.

\subsubsection{Configuration and Construction}

The dimensions of the NIST4 divider are shown in figure 50. The high voltage arm consists of a noninductive wire-wound resistor immersed in an oil-filled column, permitting a compact configuration. The high-voltage arm can be physically shorter in oil than in air since the flashover voltage along the arm when immersed in transformer oil is much higher than in air. The high-voltage arm is only $0.3 \mathrm{~m}$ in length while those of most conventional $300 \mathrm{kV}$ free-standing dividers are typically more than three times that length. The diameter of the high-voltage arm is only $2.54 \mathrm{~cm}$, which is also smaller than most other dividers having comparable voltage ratings.

There are several advantages to make the divider so compact. The compact configuration makes the high-voltage arm's stray capacitance and self-inductance much less than what is possible when air is used as the insulating medium. Lower stray capacitance gives the divider a faster transient response. Because the highvoltage arm is shorter, it has a more uniform voltage distribution along its length than otherwise possible, which provides a more linear scaling of the voltage at the divider output. 
A high voltage measuring system consists of more than just the divider, however. A high voltage lead is used to connect the test object to the divider, and this lead makes the step response for the entire measurement system slower than that for the divider alone. To reduce effects arising from other grounded or charged objects on the measurements, the IEC Standard recommends that the lead be at least as long as 1.5 times the height of the divider [103]. A shorter divider means that a shorter high voltage lead may be used without introducing significant proximity effects, and the measurement system will have a faster overall transient response.

Other advantages of using oil insulation are better heat dissipation and reduction of environmental effects. A high-voltage arm immersed in oil has much better heat conduction than one in air so that temperature effects are smaller. Leakage currents on the surface of the high-voltage arm are negligible and unaffected by humidity, since the arm is in oil. The column containing the oil and the high-voltage arm is placed or a grounded metal cylinder so that the high-voltage arm's resistance and the voltage divider ratio would be unaffected by column's surface resistance regardless of how high the humidity in the test area is.

Most external mechanical forces act on the oil-filled column instead of acting directly on the high-voltage arm itself as is the case with most free-standing resistive dividers. In this way, effects of mechanical strain on the resistance of the high-voltage arm are minimized. It is shown later in this section how important these effects are to the value of the resistance.

It is important the there be no significant corona from the divider or leads when high voltage is applied. It was found that the high-voltage arm immersed in oil was coronafree under $300 \mathrm{kV}$ impulse by observation with a micro-channel plate image intensifie: camera in a darkened room. Different high-voltage leads were also investigated in this manner. Leads of $2.54 \mathrm{~cm}$ or smaller diameter were seen to have substantial corona at that voltage. Although leads having diameters exceeding $10.2 \mathrm{~cm}$ (4 in) were seen to be corona-free, it is not convenient to use such large leads. It was found that a $1 \mathrm{~mm}$ diameter lead in an oil-filled tube was also corona-free. Use of this type of lead can provide a corona-free impulse measuring system, at least to the $300 \mathrm{kV}$ level.

A specially-designed ring is employed at the bottom of the high-voltage arm of the NIST4 divider. Unlike similar rings used for capacitive grading with most $300 \mathrm{kV}$ resistive dividers, the ring used was specifically designed to shield the lower part of the high-voltage arm to reduce noise pickup instead of providing improved voltage distribution along the high-voltage arm. It was seen from calculations based on circuit $r_{s}$. $)$ dels for $10 \mathrm{k} \Omega$ resistive dividers that high-frequency noise on the high voltage leads and top rings of the dividers should be greatly damped by the $10 \mathrm{k} \Omega$ resistance of the high-voltage arm. In step response measurements with most resistive dividers, however, significant noise still appears. It was found through comparison of diffcrent shielding configurations that most of the noise in the measured signal is picked up by the lower part of high-voltage arm where the resistance may not be high enough to 


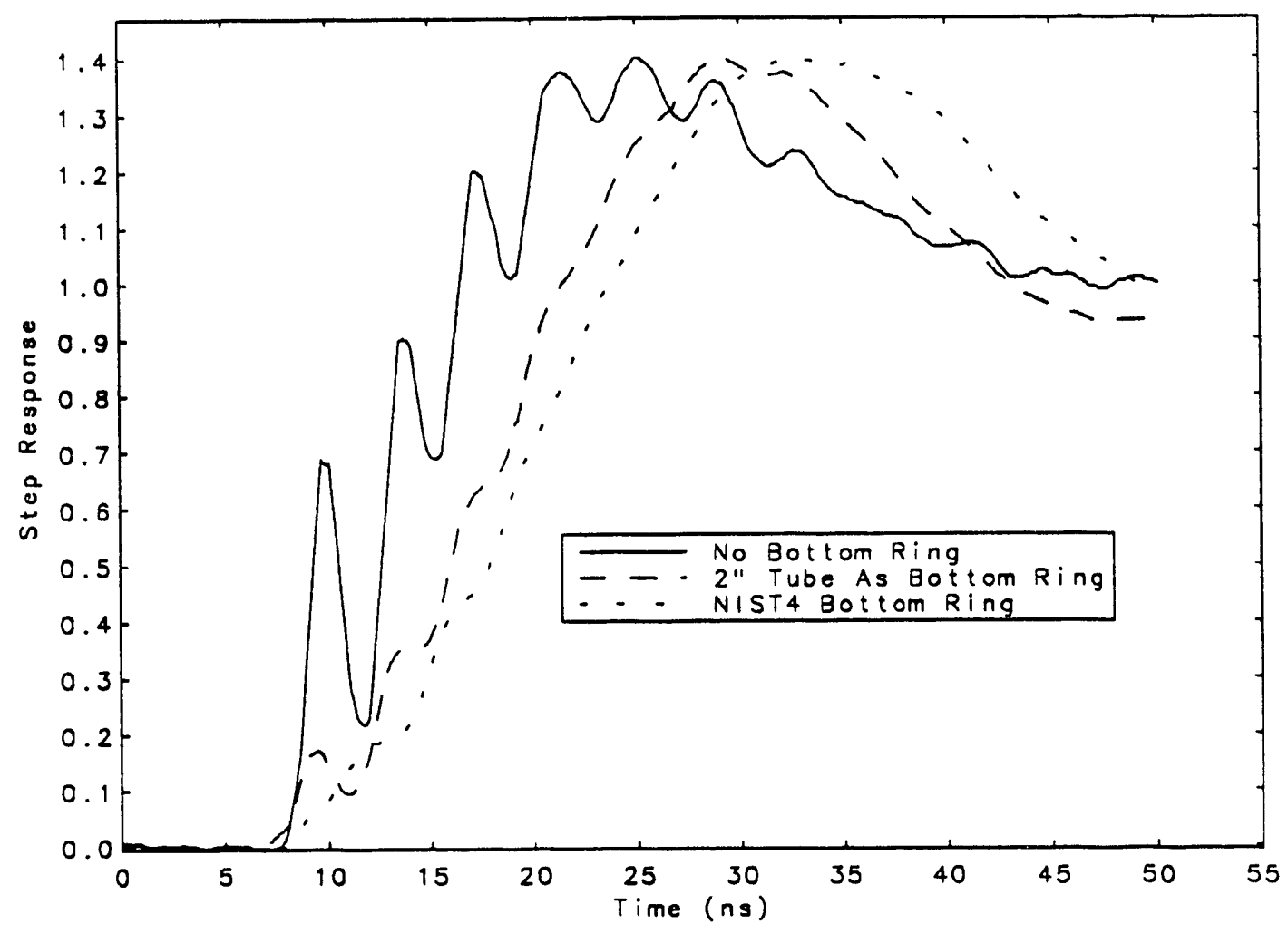

Figure 51. Step Response With and Without Bottom Rings.

attenuate radiated noise. It is therefore necessary to have an effective shielding ring at the bottom of the high-voltage arm to eliminate this noise and provide an undistorted signal. Figure 51 shows the step response with and without several different bottom rings. The final version of the ring used in the NIST4 divider was located very close to the bottom part of high-voltage arm to obtain the best shielding. A compromise was made to get both minimal signal distortion and fast response. A larger ring of a small diameter surrounding the bottom end of the high-voltage arm would have been more effective for shielding, but the increased capacitance between the high-voltage arm and the ring would produce a slower transient response. Additionally, care must be taken to avoid flashover between the ring and high-voltage arm. The ring used here was small, having an inside diameter of $5.1 \mathrm{~cm}(2 \mathrm{in})$ and a height of $5.1 \mathrm{~cm}(2 \mathrm{in})$. The NIST4 divider's step response shown in figure 52 is faster and less distorted by noise than those of the other $300 \mathrm{kV}$ free-standing dividers, such as the NIST3 divider which has a large big bottom ring of 1 meter in diameter.

There are two parts that shield the top of the NIST4 divider. One is a sphere $10.2 \mathrm{~cm}$ (4 in) in diameter on top of the divider that is used to reduce electrical field stresses and eliminate corona. The second shield is a copper cap located inside the oil column to provide the fast response and better voltage distribution along the high-voltage arm. The cap was empirically designed so that the fastest step response possible was achieved with acceptable overshoot. The edge of the cap was made of a $1.27 \mathrm{~cm}$ 


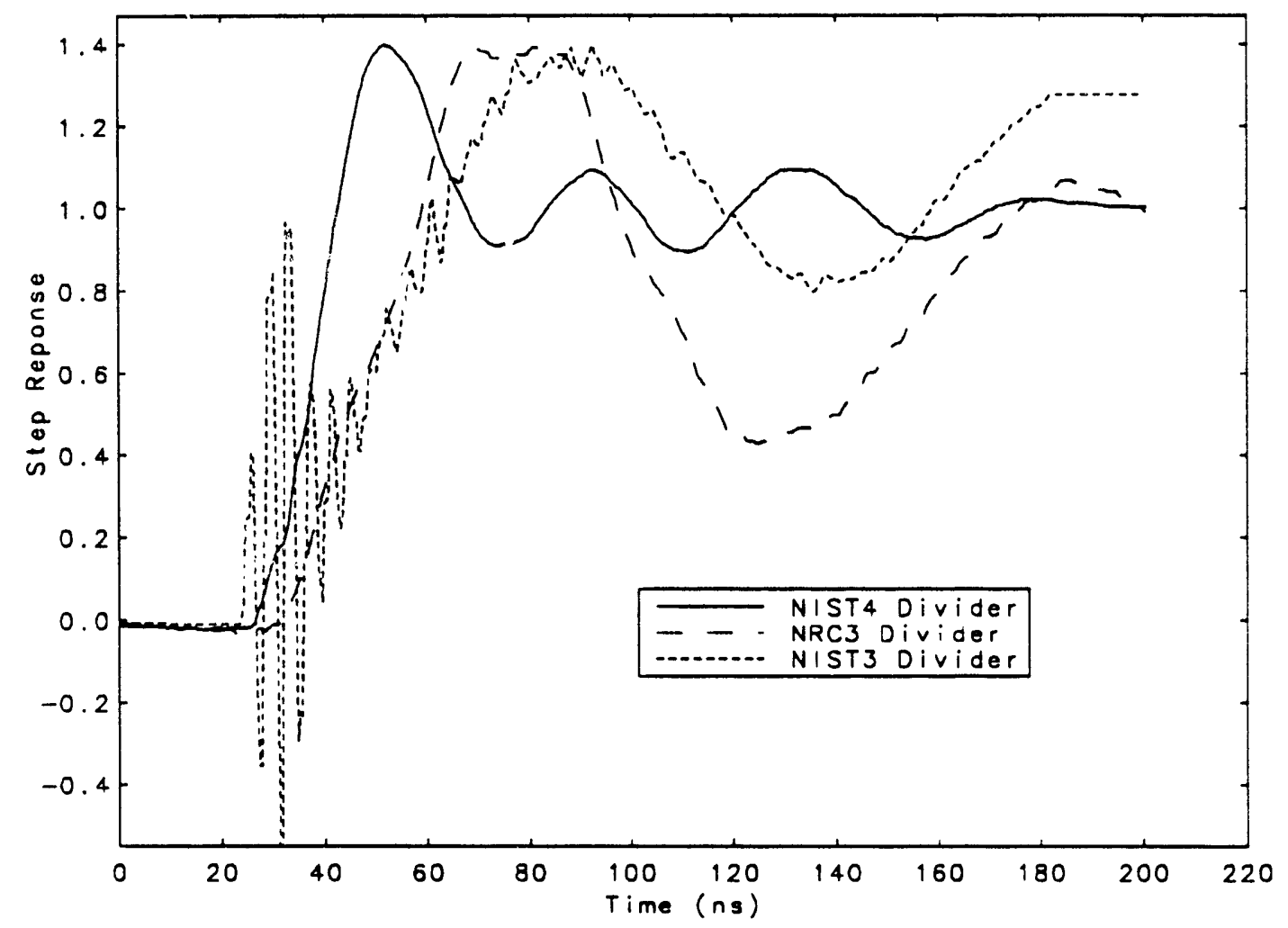

Figure 52. NIST4 Step Response. 
( 0.5 in) diameter copper tube to ensure that corona would not be produced at the rated voltage.

The resistive wire used in the high-voltage arm of the NIST4 divider was an alloy-type wire having a temperature coefficient of $-3 \mathrm{ppm}$ per degree centigrade. Its diameter of $0.11 \mathrm{~mm}(0.0045 \mathrm{in})$ is thicker than that used in most other resistive dividers, giving the high-voltage arm a greater heat capacity and lower temperature increase after application of high voltage impulses. The low temperature coefficient, larger diameter of the wire, and the thermal conductivity of the oil keep the divider's resistance very stable during environmental temperature changes or when several applications of full lightning impulses are macie. The resistance of the high-voltage arm was measured wich a resistance bridge to be $10.074 \mathrm{k} \Omega$ at room temperature. Immediately following the application of ten consecutive standard lightning impulses of $325 \mathrm{kV}$ peak voltage over a period of 75 seconds, the measured resistance was $10.075 \mathrm{k} \Omega$. The difference in resistance can be considered a negligible change. The low-voltage arm's measured resistance had the same value of $12.513 \Omega$ both before and after the ten impulses.

The temperature coefficient of resistance for the high-voltage arm depends not only upon heating of the wire but also upon the changing mechanical stresses of the wire. An interesting experiment investigating temperature and mechanical effects was conducted. Three sample resistórs of the same diameter were made with resistive wire and placed. into an oven. The first sample was a simple wire loop. The second was wound on a polyethylene rod and the third was wound on a machinable glass ceramic rod. Table 1 shows the change in resistance after heating the resistors from $20^{\circ} \mathrm{C}$ to $60^{\circ} \mathrm{C}$. The resistance of the sample wound on the polyethylene rod had been changed $0.08 \%$ after heating to $41^{\circ} \mathrm{C}$. It is believed that this is not due to the temperature coefficient of the winding, but rather to the thermal expansion of the rod during heating which resulted in a strain-induced resistance change. Changes in resistance of this magnitude are unacceptable for reference measurement systems. The machinable glass ceramic had a very good resistance stability at high temperature. Table 2 shows the linear thermal coefficient of expansion for a few materials that are candidates for substrates on which to wind the resistive wire. The glass ceramic has an expansion coefficient ten times smaller than the other three commonly-used materials. As a result, it was selected for NIST4 divider as a base for the high-voltage arm.

The high-voltage arm was wound in the typical manner for resistive dividers. Two layers of windings are counterwound to minimize inductance. The inner one is wound in clockwise fashion and the outer one in counterclockwise fashion. The difference between the windings of the NIST4 divider and most air-insulated dividers is that the rod on which the wire is wound has a much smaller diameter and separation between turns. There are 65.7 turns per centimeter (167 turns per inch). From prior experience[110], it is known that the smaller the rod diameter and the more closer the spacing of the windings are, the smaller the net magnetic flux produced by the two windings will be. These are important considerations in minimizing the residual inductance of the wound resistor. The windings begin on a brass end cap and 
Table 1. Resistance Change of Resistor Samples During Heating.

$\begin{array}{cccc}\mathrm{T}\left({ }^{\circ} \mathrm{C}\right) & \text { Simple } & \begin{array}{c}\text { Polyethylene } \\ \operatorname{Rod}(\Omega)\end{array} & \begin{array}{c}\text { Glass Ceramic } \\ \operatorname{Rod}(k \Omega)\end{array} \\ 20 & 0.59433 & 2.5204 & \\ 41 & - & 2.5224 & 10.0777 \\ 62 & 0.59431 & - & - \\ & & & 10.0784\end{array}$

Table 2. Linear Thermal Coefficients of Expansion of Candidate Substrates.

Polyethylene Polytetrafluoroethylene Nylon Glass Ceramic
$1.7 \times 10^{-4}$
$1.0 \times 10^{-4}$
$1.5 \times 10^{-4}$
$9.4 \times 10^{-6}$

continue along the ceramic rod to the opposite brass end cap. After the first layer was wound, an epoxy coating was applied to provide insulation between the windings. The second layer of wire was then counterwound on top of the first. The epoxy layer used between the windings is thin, compatible with transformer oil, and long-lasting at temperatures below $105^{\circ} \mathrm{C}$. Since it is thin, tise diameters of the inner and outer windings are nearly the same, which in'so helps to reduce the overall inductance of the resistor. After both windings were complete, their ends were secured with screws to the brass end caps to ensure that there is no mechanical stress on the wires caused by moving the divider.

The low-voltage arm of the NIST4 divider has low inductance, high wattage, and good shielding. It comprises sixteen $200 \Omega, 1 \mathrm{~W}$ tin oxide resistors in parallel. There are two shields for the low-voltage arm, an inner cylindrical copper shield and an outer steel cylinder. Together they provide excellent shielding to electromagnetic interference. The measured step response for the low-voltage arm is shown in figure 53, from which the risetime is calculated to be less than $1 \mathrm{~ns}$.

\subsection{The Unit Step Response (USR) and Its Application}

\subsubsection{Step Response Measurement}

Step responses of the NIST4 divider were taken using different damping resistors in the circuit. The geometry used for the step response measurements is the horizontal loop configuration shown schematicaliy in figure 54 . In this configuration, the mercury-wetted relay used to generate the voltage step is mounted on a support 


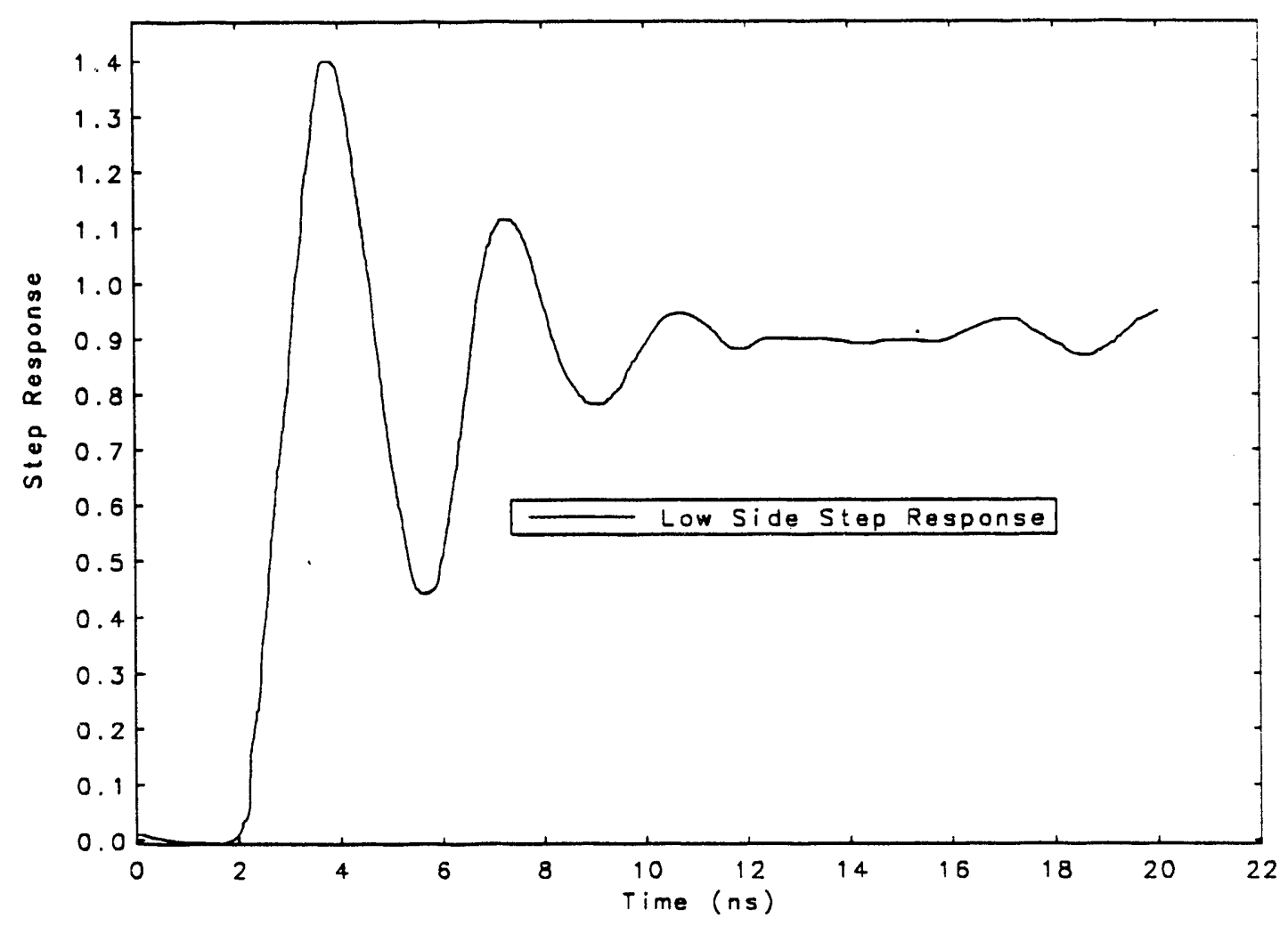

Figure 53. Step Response of the Low-Voltage Side of NIST4 


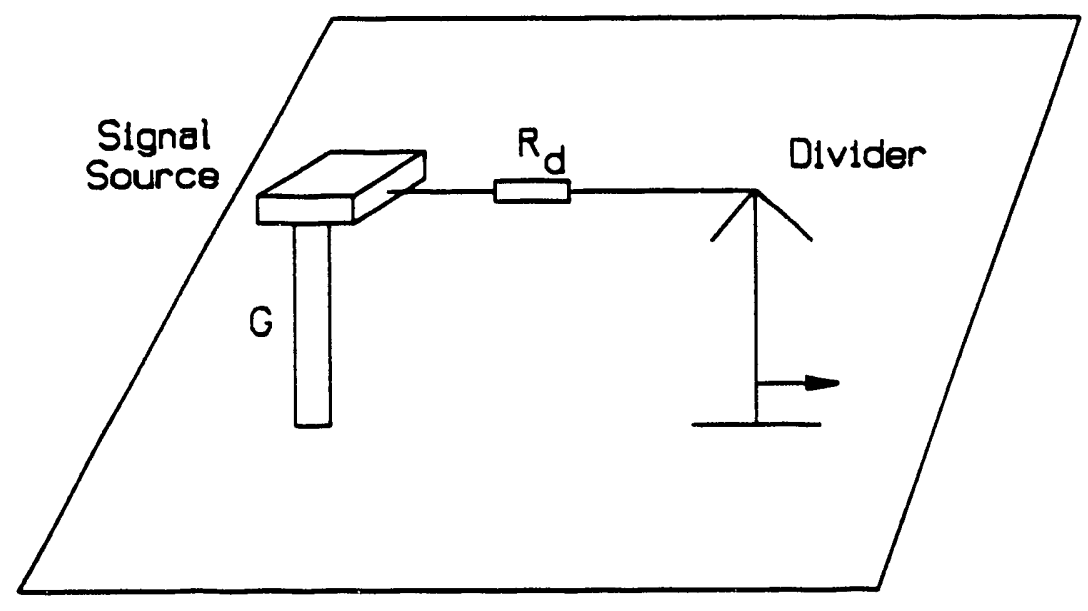

Figure 54. Horizontal Loop Configuration for Step Response Measurement

at a height of about $1 \mathrm{~m}$ above the ground plane and connected to ground with a wide copper strip labeled $\mathrm{G}$ in the figure. As mentioned previously, the step response is changed by the diameter and length of the leads connecting the step generator to the divider. A comparison of the step responses for several different leads is shown in figure 55. As seen from the figure, the thinner and longer the lead, the worse the step response, having longer risetimes, greater overshoot, and more oscillations.

Table 3 compares the step response parameters $T_{n}$ (experimental response time), $T_{\alpha}$ (partial response time), and $T_{\circ}$ (initial distortion time) for the NIST4, NIST3, and NIST1 dividers, taken with different damping resistances, $R_{d}$. 


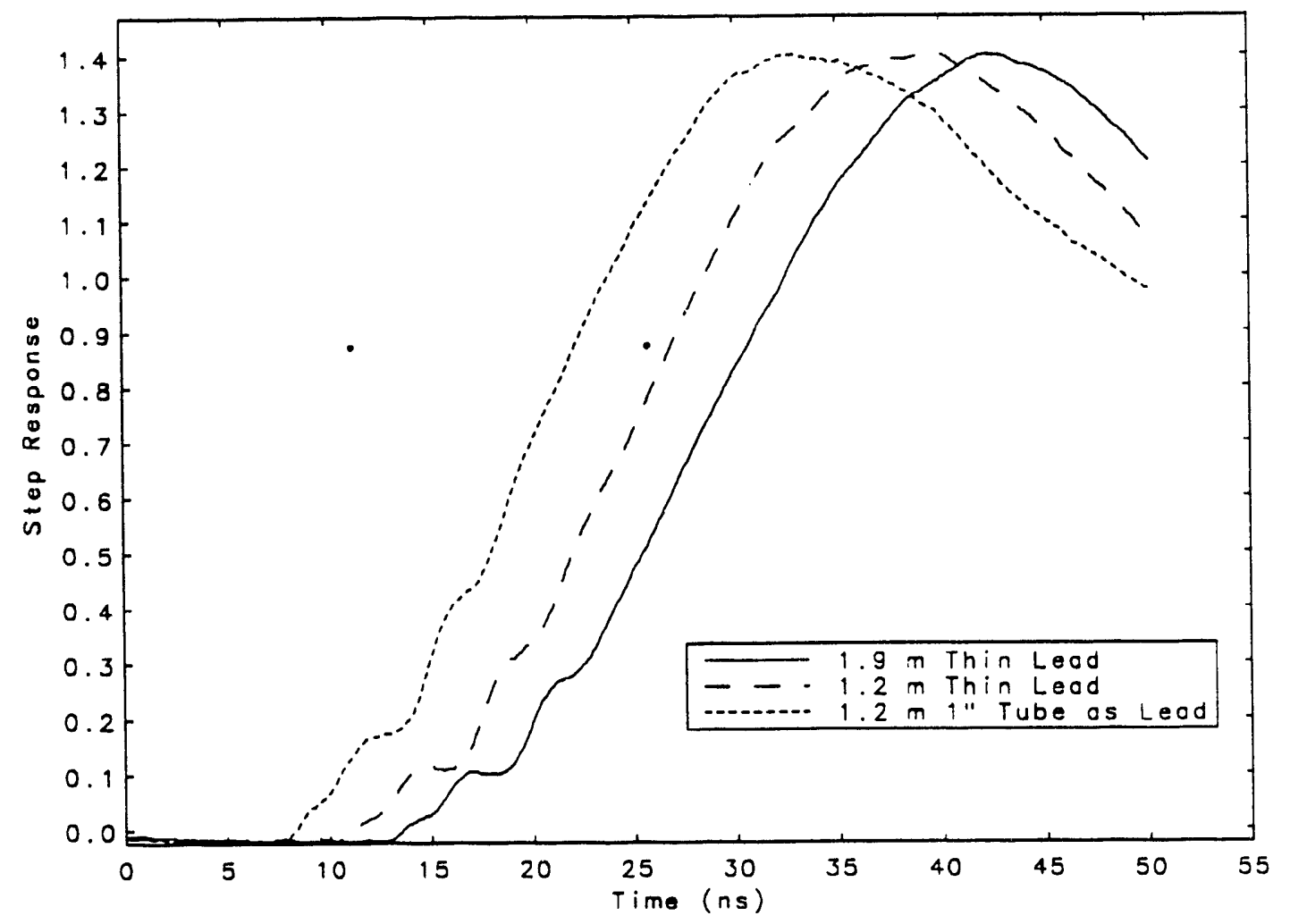

Figure 55. Comparison of Step Responses for Different Lead Lengths 


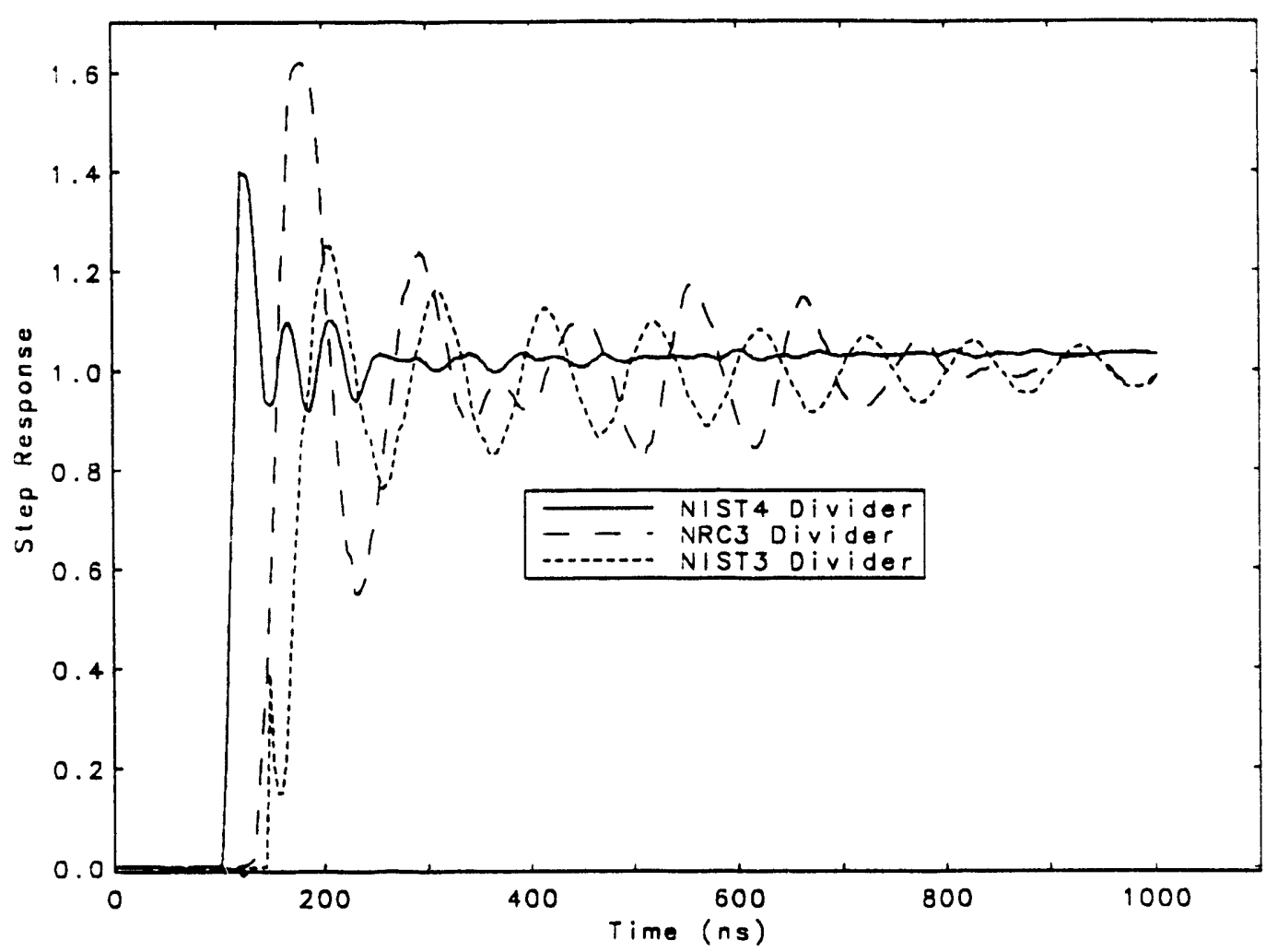

Figure 56. Step Responses of NIST4, NIST3, and NIST1

The NIST3 and NIST1 dividers are conventional air-insulated, free-standing resistive devices rated for $300 \mathrm{kV}$ and $1 \mathrm{MV}$ impulses, respectively. Figure 56 shows a comparison of the step responses for these dividers. Compared with other $300 \mathrm{kV}$ dividers used in reference systems such as NIST3, the NIST4 divider's step response was much faster and less distorted. For the undamped cases, (i.e., no external damping resistor in the step response circuit), the 10\%-90\% risetime of the step responses for the three dividers is summarized in table 4. The NIST4 divider clearly has a much-improved risetime.

\subsubsection{Convolution Calculation}

The convolution technique can be used with the measured step response to predict the output of an impulse measurement system for an assumed analytical or numerical input. This approach, performed in the time domain, has been presented previously [111]. A frequency-domain convolution method for estimating measurement errors is now described.

For a linear system with an input step voltage $L(t)$ and an impulse response $H(t)$, the step response $G(t)$ is:

$$
G(t)=H(t) \neq L(t)
$$


Table 3. Step Response Parameters for Three Reference Dividers

\begin{tabular}{|l|r|c|c|c|c|}
\hline Divider & $\begin{array}{c}\mathrm{R}_{d} \\
(\Omega)\end{array}$ & $\begin{array}{c}\mathrm{T}_{n} \\
(\mathrm{~ns})\end{array}$ & $\begin{array}{c}\mathrm{T}_{\alpha} \\
(\mathrm{ns})\end{array}$ & $\begin{array}{c}\mathrm{T}_{\circ} \\
(\mathrm{ns})\end{array}$ & $\begin{array}{c}\text { Overshoot } \\
(\%)\end{array}$ \\
\hline NIST4 & 0 & 0.3 & 5.3 & 0.6 & 37.5 \\
& 250 & 5.5 & 8.8 & 0.6 & 15.2 \\
& 500 & 14 & 14.3 & 0.2 & 1.7 \\
\hline NIST3 & 0 & 19 & 17 & 2.6 & 24 \\
& 150 & 23 & 20 & 1.8 & 11 \\
& 350 & 28 & 29 & 1.3 & 3 \\
& 550 & 36 & 36 & 0.8 & 1 \\
\hline NIST1 & 0 & 23 & 27 & 2.9 & 39 \\
& 150 & 33 & 36 & 2.7 & 14 \\
& 250 & 41 & 44 & 1.7 & 6 \\
& 400 & 52 & 53 & 1.8 & 2 \\
\hline
\end{tabular}

Table 4. Rise Time (ns) Comparison for Three Reference Dividers

\begin{tabular}{|c|c|c|}
\hline NIST4 & NIST3 & NIST1 \\
\hline 11 & 40 & 52 \\
\hline
\end{tabular}


Table 5. Calculated Peak Voltage Outputs of NIST4 Divider

\begin{tabular}{|r|c|c|c|}
\hline $\begin{array}{r}R_{d} \\
(\Omega)\end{array}$ & $\begin{array}{c}\text { Full lightning } \\
\text { (front-time } \\
0.915 \mu \mathrm{s})\end{array}$ & $\begin{array}{c}\text { Chopped lightning } \\
\text { (Chopped at 88\% } \\
\text { of the peak, Time- } \\
\text { to-chop 0.566 } \mu \mathrm{s})\end{array}$ & $\begin{array}{c}\text { Chopped lightning } \\
\text { (Chopped at 98\% } \\
\text { of the peak, Time- } \\
\text { to-chop 0.957 } \mu \mathrm{s})\end{array}$ \\
\hline 0 & 1.0000 & 0.9991 & 0.9993 \\
\hline 250 & 1.0000 & 0.9948 & 0.9990 \\
\hline 500 & 1.0000 & 0.9914 & 0.9983 \\
\hline
\end{tabular}

where the "*" represents the convolution integral. Similarly, for an input signal $\mathrm{X}(\mathrm{t})$ the corresponding output signal $\mathrm{Y}(\mathrm{t})$ is:

$$
Y(t)=H(t) * X(t)
$$

In frequency domain, if $G(t), L(t), H(t), X(t)$, and $Y(t)$ have Fourier transforms $G(f)$, $\mathrm{L}(\mathrm{f}), \mathrm{H}(\mathrm{f}), \mathrm{X}(\mathrm{f}), \mathrm{Y}(\mathrm{f})$ respectively, then

$$
\begin{aligned}
& G(f)=H(f) \cdot L(f) \\
& Y(f)=H(f) \cdot X(f)
\end{aligned}
$$

From (16) and (17),

$$
Y(f)=\frac{X(f) \cdot G(f)}{L(f)}
$$

From this formula, if a system's step response is known, one can predict an output for any given input signal by taking the inverse Fourier transform of equation (18). Full standard lightning impulses along with the chopped lightning impulses shown in figure 57 were used as input signals to calculate corresponding output of the NIST4 divider. Table 5 gives some of the peak values of the output waveforms for normalized inputs.

The data of table 5 inciicate that the NIST4 divider can measure the peak voltage of full lightning impulses with negligible error. The data also show that the NIST4 divider has less than $1 \%$ error in peak voltage measurement of front-chopped lightning impulses. 

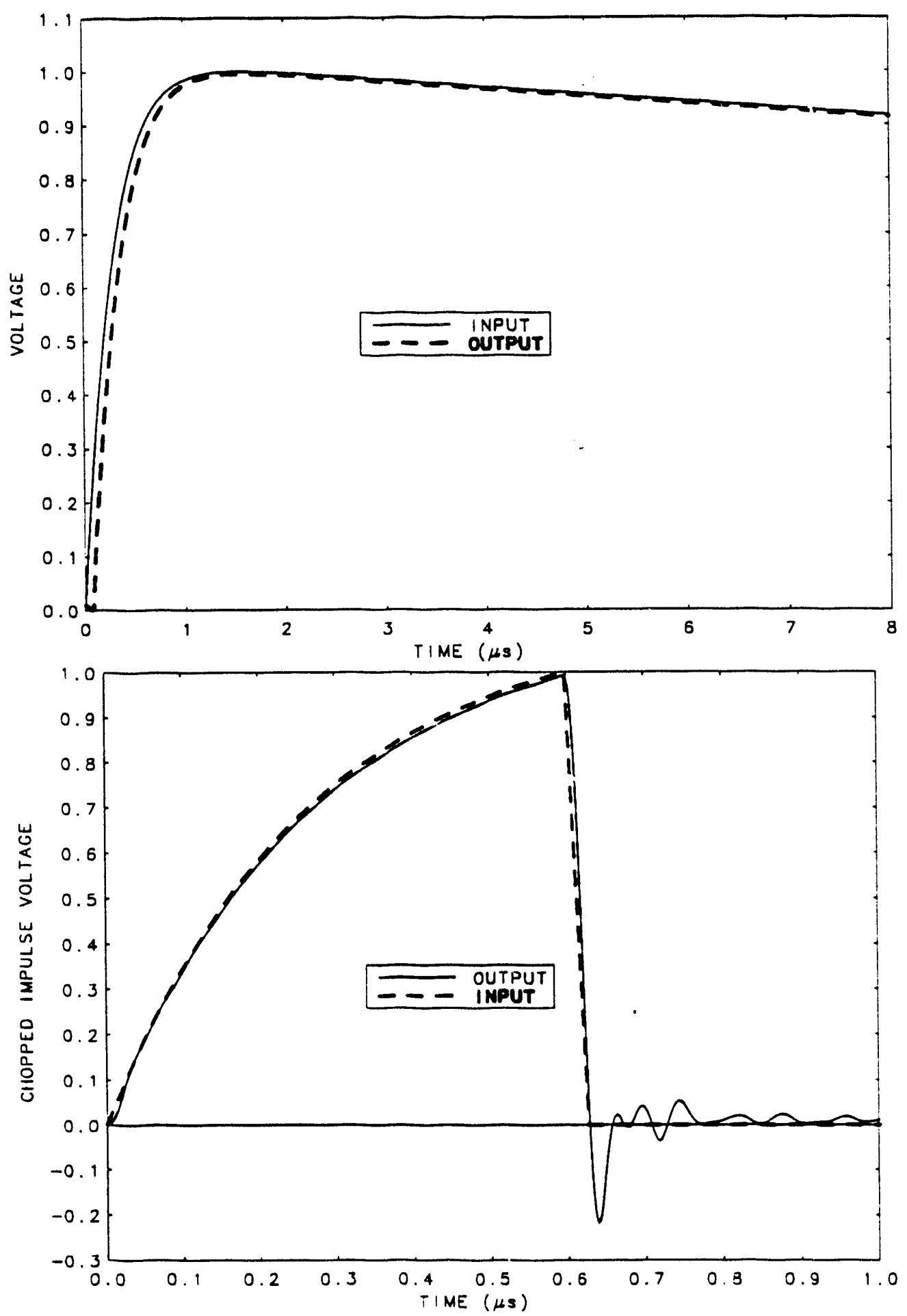

Figure 57. Typical (a) Full and (b) Chopped Lightning Impulses 


\subsection{Conclusion}

A new, compact resistive voltage divider has been designed, constructed, and characterized for use as part of an impulse voltage measurement system. Although rated for impulse voltages up to $300 \mathrm{kV}$, it stands only $0.8 \mathrm{~m}$ tall. It has significant advantages over other free-standing dividers because of its small physical size, oil insulation, and shielding. The small physical size gives it a response time that is nearly a factor of four times faster than references dividers with comparable voltage ratings while the oil insulation provides much better insensitivity to environmental effects, such as temperature changes and humidity than air-insulated dividers do. A specially-designed shield for the bottom of the nigh-voltage side of the divider further reduces distortion of the divider output by minimizing the pickup of spurious signals. The divider's measured step response of $10 \mathrm{~ns}$ is much faster than comparable free-standing dividers. The NIST4 divider represents significant improvement in the measurement of high voltage standard lightning impulses with free-standing resistive voltage dividers. 


\section{REFERENCES}

[1] M. Misakian and W. T. Kaune, "An Optimum Experimental Design For In Vitro Studies Using ELF Magnetic Fields," Bioelectromagnetics, Vol. 11, pp. 251-255 (1990).

[2] D. L. Miller, M. C. Miller, and W. T. Kaune, "Addition of Magnetic Capability to Existing Extremely-Low-Frequency Electric Field Exposure Systems," Bioelectromagnetics, Vol. 10, pp. 85-88 (1989).

[3] B. R. McLeod, A. A. Pilla, and M. W. Sampsel, "Electromagnetic Fields Induced by Helmholtz Aiding Coils Inside Saline-Filled Boundaries", Bioelectromagnetics, Vol. 4, pp. 357-370 (1983).

[4] M. M. Cohen, S. Schwartz, A. Kunska, J. Satish, and A. Hamburger, "The Effect of Tissue Culture Agar on Chromosome Breakage, Sister Chromatid Exchanges and Clonogenicity in Human Cells", Mutat. Res., Vol. 208, pp. 201205 (1988).

[5] D. Krause, private communication.

[6] The theory of calculating current distributions in flat conductors that undergo a sudden change in width is described by Smythe [W.R. Smythe, Static and Dynamic Electricity, pp. 254-256 (McGraw-Hill, New York, 1968)] using a conformal mapping technique. Figure 9 shows a calculated current distribution assuming the conductor width changes by a factor of four.

[7] H. B. Dwight, "Skin Effect in Tubular and Flat Conductors", AIEE Trans., Vol. 37, pp. 1374-1400 (1918).

[8] M. Misakian, "Electrical Parameters in 60-Hz Biological Exposure Systems and Their Measurement: A Primer," NBS Technical Note 1191 (1984).

[9] J. K. Olthoff, R. J. Van Brunt, J. T. Herron, and I. Sauers, "Detection of Trace Disulfur Decafluoride in Sulfur Hexafluoride by Gas Chromatography/Mass Spectrometry", Annl. Chem., Vol. 63, pp. 726-732 (1991).

[10] J. K. Olthoff, R. J. Van Brunt, J. T. Herron, I. Sauers, and G. Harman, "Catalytic Decomposition of $\mathrm{S}_{2} \mathrm{~F}_{10}$ and its Implications on Sampling and Detection from $\mathrm{SF}_{6}$-Insulated Equipment", Conf. Rec. 1990 IEEE Int. Symp. on Elec. Insul., IEEE Publ. 90 CH2727-6, pp. 248-252 (1990).

[11] I. Sauers, G. Harman, J. K. Olthoff, and R. J. Van Brunt, " $\mathrm{S}_{2} \mathrm{~F}_{10}$ Formation by Electrical Discharges in $\mathrm{SF}_{6}$ : Comparison of Spark and Corona", Proceedings of the 6th International Symposium on Gaseous Dielectrics, Gaseous Dielectrics VI, (L. G. Christophorou and I. Sauers, Eds.) Plenum Press, New York, pp. 528-537 (1991). 
[12] R. J. Van Brunt and S. V. Kulkarni, "Stochastic Properties of Trichel-Pulse Corona: A Non-Markovian Random Point Process", Phys. Rev. A, Vol. 42, pp. 4908-4932 (1990).

[13] S. V. Kulkarni, R. J. Van Brunt, and V. K. Lakdawala, "Transition from Trichel-Pulse Corona to Dielectric-Barrier Discharge", Annual Report 1990 IEEE Conference on Electrical Insulation and Dielectric Phenomena, Pocono Manor, PA, pp. 267-274 (1990).

[14] R. J. Van Brunt and S. V. Kulkarni, "Influence of Memory on the Statistics of Pulsating Corona", Pruceedings of the 6th International Symposium on Gaseous Dielectrics, Gaseous Dielectrics VI, (L. G. Christophorou and I. Sauers, Eds.) Plenum Press, New York (1991).

[15] R. J. Van Brunt, K. L. Stricklett, J. P. Steiner and S. V. Kulkarni, "Recent Advances in Partial Discharge Measurement Capabilities at the National Institute of Standards and Technology", IEEE Trans. Elec. Insul. (submitted, 1991).

[16] R. J. Van Brunt and E. W. Cernyer, "Influence of Melıory Propagation on Phase-Resolved Stochastic Behavior of AC-Generated Partial Discharges", Appl. Phys. Lett. (submitted, 1991).

[17] R. J. Van Brunt, M. Misakian, S. V. Kulkarni, and V. K. Lakdawala, "Influence of a Dielectric Barrier on the Stcchastic Behavior of Trichel-Pulse Corona", (submitted, 1991).

[18] L. A. Greenberg and D. Lester, "the Toxicity of Sulfur Pentafluoride" Arch. of Ind. Hyg. Occupational Med., Vol. 2, pp. 350-353 (1950).

[19] J. P. Saunders, M. M. Shoskes, M. R. DeCario, and E. C. Brown, "Some Physiological Effects of Disulfur Decafluoride after Intravenous Injection in Dogs", Arch. of Ind. Hyg. Occupational Med., Vol. 8, pp. 436-445 (1953).

[20] G. D. Griffin, I. Sauers, K. Kurka, and C. E. Easterly, "Spark Decomposition of $\mathrm{SF}_{6}$ : Chemical and Biological Studies", IEEE Trans. Power Delivery, Voi. 4, pp. 1541-1551 (1989).

[21] G. D. Griffin, M. G. Nolan, I. Sauers, K. Kurka, M. D. Morris, and P. C. Votaw, "Cytotoxic Activity of Disulfur Decafluoride $\left(\mathrm{S}_{2} \mathrm{~F}_{10}\right)$, A Decomposition Product of Electricity-Stressed $\mathrm{SF}_{6}{ }^{\prime}$, In Vitro, Vol. 25, pp. 673-675 (1989).

[22] American Conference of Governmental and Industrial Hygienists (ACGIH) (1986). Documentation of Threshold Limit Values and Biological Exposure Indices, 5th Ed., American Conference of Industrial Hygienists, Inc. Cincinnati, $\mathrm{OH}$ p. 545. 
[23] I. Sauers, P. C. Votaw, and G. D. Griffin, "Production of $S_{2} F_{10}$ in Sparked $\mathrm{SF}_{6}$, ” J. Phys. D: Appl. Phys., Vol. 21, pp. 1236-1238 (1988).

[24] I. Sauers, M. C. Siddagangappa, G. Harman, R. J. Van Brunt, and J. T. Herron, "Production and Stability of $\mathrm{S}_{3} \mathrm{~F}_{10}$ in $\mathrm{SF}_{6}$ Corona Discharges", Proc. of sixth Int. Symp. on High Voltage Engineering (New Orleans), Vol. 1, paper 23.08 (1989).

[25] J. T. Herron, "S $\mathrm{S}_{2} \mathrm{~F}_{10}$ Formation in Computer Simulation Studies of the Breakdown of SF ${ }_{6}$, IEEE Trans. Elec. Insul., Vol. EI-22, pp. 523-525 (1987).

[26] R. J. Van Brunt, and J. T. Herron, "Fundamental Processes of SF 6 Decomposition and Oxidation in Glow and Corona Discharges", IEEE Trans. Elec. Insul., Vol. 25, pp. 75-94 (1990).

[27] Federal Register, Air Contaminants Final Rule, OSHA, Jan. 19 (1989).

[28] F.J.J.G. Janssen, "Measurement at sub-ppm Level of Sulfur-Fluoride Compounds Resulting from the Decomposition of $\mathrm{SF}_{6}$ by Arc Discharges", Kema Sci. Tech. Reports, Vol. 2, pp. 9-18 (1984).

[29] F.J.J.G. Janssen, Decomposition of $\mathrm{SF}_{6}$ by Arc Discharge and the Determination of the Reaction Product $\mathrm{S}_{2} \mathrm{~F}_{10}$ ", Proceedings of the 5th International Symposium on Gaseous Dielectrics V, (L. G. Christophorou and D. W. Bouldin, Eds.) Pergamon Press, pp. 153-162 (1987).

[30] B. Cohen and A. G. MacDiarmid, Inorga. Chem., Vol. 1, pp. 754-756 (1962).

[31] J. K. Olthoff, R. J. Van Brunt, I. Sauers, "Electron-Energy Dependence of the $\mathrm{S}_{2} \mathrm{~F}_{10}$ Mass Spectrum", J. Phys. D: Appl. Phys., Vol. 22, pp. 1399-1401 (1989).

[32] M. Farber, S. P. Cooper, and M. Khazei, " Mass Spectrometric Determination of $\mathrm{S}_{2} \mathrm{~F}_{10}$ Resulting from High-Voltage Arcing of $\mathrm{SF}_{6}$ ", J. Phys. D: Appl. Phys., Vol. 22, pp. 233-234 (1989).

[33] J. M. Hamraham and A. R. Patterson, "Adsorption-Desorption Gas Chromatographic Infrared Determination of Trace Sulfur Decafluoride in Sulfur Hexafluoride", J. Chromat., Vol. 193, pp. 265-275 (1980).

[34] R. J. Van Brunt, "Production Rates for Oxyfluorides $\mathrm{SOF}_{2}, \mathrm{SO}_{2} \mathrm{~F}_{2}$, and $\mathrm{SOF}_{4}$ in $\mathrm{SF}_{6}$ Corona Discharges", J. Res. Nat. Bur. Stand., Vol. 90, pp. 229-253 (1985).

[35] R. J. Van Brunt and M. C. Siddagangappa, "Identification of Corona DischargeInduced $\mathrm{SF}_{6}$ Oxidation Mechanisms Using $\mathrm{SF}_{6} /{ }^{18} \mathrm{O}_{2} / \mathrm{H}_{2}^{16} \mathrm{O}$ and $\mathrm{SF}_{6} /{ }^{16} \mathrm{O}_{2} / \mathrm{H}_{2}^{18} \mathrm{O}$ Gas Mixtures, Plasma Chem. Proc., Vol. 8, pp. 207-223 (1988). 
[36] R. Hergli, J. Casanovas, A Derouri, R. Grob, and J. Mathieu, "Study of the Decomposition of $\mathrm{SF}_{6}$ in the Presence of Water, Subjected to Gamma Irradiation or Corona Discharges", IEEE Trans. Elec. Insul., Vol. 23, p. 451 (1988).

[37] V. H. Dibeler and F. L. Mohler, J. Res. Nat. Bur. Stand., Vol. 40, pp. 25-33 (1948).

[38] A. Cornu and R. Massot, Compilation of Mass Spectra Data, 2nd Edition, (Heyden and Sons, NY) p. 77A (1975).

[39] W. R. Trost and R. L. McIntosh, "The Kinetics of the Thermal Decomposition of Disulfur Decafluoride”, Can. J. Chem., Vol. 29, pp. 508-525 (1952).

[40] S. W. Benson and J. Bott, Int. J. Chem. Kinetics, Vol. 1 pp. 451-458 (1969).

[41] J. T. Herron, "A Critical Review of the Chemical Kinetics of $\mathrm{SF}_{4}, \mathrm{SF}_{5}$, and $\mathrm{SF}_{2} \mathrm{~F}_{10}$ in the Gas Phase", Int'l. J. Chem. Kinet., Vol. 19, pp. 129-142 (1987).

[42] .J. C. Tait and J. A. Howard, Can. J. Chem., Vol. 53, pp. 2361-2362 (1975).

[43] R. J. Van Brunt and I. Sauers, "Gas-Phase Hydrolysis of SOF 2 and $\mathrm{SOF}_{4}$ ", J. Chem. Phys., Vol. 85, pp. 4377-4380 (1986).

[44] M. D. Vorbev, A. S. Filatov, and M. A. Englin, J. Gen. Chem. USSR, Vol. 44, pp. 2677-2679 (1975).

[45] H. L. Roberts, J. Chem. Soc., pp. 3183-3185 (1962).

[46] H. W. Sidebottom, J. M. Tedder, and J. C. Walton, Trans. Faraday Soc., Vol. 65, pp. 2103-2109 (1969).

[47] B. Cohen and A. G. MacDiarmid, Inorgan. Chem., Vol. 4, pp. 1782-1785 (1965).

[48] R. Bartnikas, "Detection of Partial Discharges (Corona) in Electrical Apparatus," IEEE Trans. Elec. Insul., Vol. EI-25, pp. 111-124 (1990).

[49] L. B. Loeb, “Electrical Coronas-Their Basic Physical Mechanisms," University of California Press, pp. 26-40 (1965).

[50] R. S. Sigmond, "Corona Discharges," in Electrical Breakdown of Gases, Ed. by J. M. Meek and J. D. Craggs, John Wiley and Sons, pp. 319-384 (1978).

[51] R. Bartnikas, "A Commentary on Partial Discharge Measurement and Detection," IEEE Trans. Elec. Insul., Vol. EI-22, pp. 629-653 (1987).

[52] F. H. Kreuger, "Discharge Detection in High Voltage Equipment," Elsevier, New York (1965). 
[53] R. Bartnikas, "Use of Multichannel Analyzers for Corona Pulse Height Distribution Measurements in Cables and Other Electrical Apparatus," IEEE Trans. on Instrum. and Measurement, Vol. 22, pp. 403-407 (1973).

[54] R. Bartnikas, "Corona Pulse Counting and Pulse Height Analysis Techniques," in Engineering Dielectrics, Vol. 1, eds. R. Bartnikas and E. J. McMahon, STP 669; ASTM Press, Philadelphia, pp. 285-326 (1979).

[55] R. J. Van Brunt and D. Leep, "Characterization of Point-Plane Corona Pulses in $\mathrm{SF}_{6}$," J. Appl. Phys., Vol. 52, pp. 6588-6600 (1981).

[56] S. V. Kulkarni and R. S. Nema, "Broad Band Pulse Detection Studies of Corona and Breakdown in Air, $\mathrm{N}_{2}, \mathrm{O}_{2}, \mathrm{CO}_{2}, \mathrm{SF}_{6}$, and $\mathrm{SF}_{6}-\mathrm{N}_{2}$ Mixtures," in Gaseous Dielectrics V, Ed. by L. G. Christophorou and D. W. Bouldin, Pergamon Press, New York, pp. 637-642 (1987).

[57] R. J. Van Brunt and M. Misakian, "Mechanisms for Inception of DC and $60 \mathrm{~Hz}$ AC Corona in SF 6 ," IEEE Trans. Elec. Insul., Vol. EI-17, pp. 106-120 (1982).

[58] H. Hikita, Y. Yamada, A. Nakamura, T. Mizutani, A. Oohasi, and M. Ieda, "Measurement of Partial Discharges by Computer and Analysis of Partial Discharge Distribution by the Monte Carlo Method," IEEE Trans. Elec. Insul., Vol. 25, pp. 453-468 (1990).

[59] J. Fuhr, M. Haessig, B. Fruth, and T. Kaiser, "P-D-Fingerprints of Some High Voltage Apparatus," Conference Record of the 1990 IEEE International Symposium on Electrical Insulation, Toronto, Canada, pp. 129-132 (1990).

[60] R. J. Van Brunt and S. V. Kulkarni, "Method for Measuring the Stochastic Properties of Corona and Partial-Discharge Pulses," Rev. Sci. Instrum., Vol.60, pp. 3012-3023 (1989).

[61] R. J. Van Brunt and S. V. Kulkarni, "New Method for Measuring the Stochastic Properties of Corona and Partial Discharge Pulses," Conference Record of the 1988 IEEE International Symposium on Electrical Ins?! ation, New York, NY pp. 233-237 (1988).

[62] S. V. Kulkarni and R. J. Van Brunt, "Stochastic Properties of Negative Corona (Trichel) Pulses in $\mathrm{SF}_{6} / \mathrm{O}_{2}$ Mixtures," Proceedings Ninth International Conference on Gas Discharges and Their Applications, Benetton, Editore, Padova, pp. 227-230 (1988).

[63] D. L. Snyder, Random Point Processes, John Wiley and Sons, New York (1975).

[64] J. P. Steiner, "Digital Measurement of Partial Discharge," Ph.D. Thesis, Purdue University, W. Lafayette, IN, May (1988). 
[65] G. W. Trichel, "The Mechanism of the Negative Point to Plane Corona Near Onset," Phys. Rev., Vol. 54, pp. 1078-1084 (1938).

[66] L. B. Loeb, A. F. Kip, G. G. Hudson, and W. H. Bennett, "Pulses in Negative Point-to-Plane Corona," Phys. Rev., Vol. 60, pp. 714-722 (1941).

[67] R. Morrow, "Theory of Stepped Pulses in Negative Corona Discharges," Phys. Rev. A, Vol. 32, pp. 3821-3824 (1985).

[68] J. A. Cross, R. Morrow and G. N. Haddad, "Negative Point-Plane Corona in Oxygen," J. Phys. D: Appl. Phys., Vol. 19, pp. 1007-1017 (1986).

[69] D. A. Scott and G. N. Haddad, "Negative Corona in Nitrogen-Oxygen Mixtures," J. Phys. D: Appl. Phys., Vol. 20, pp. 1039-1044 (1987).

[70] W. L. Lama and C. F. Gallo, "Systematic Study of the Electrical Characteristics of the 'Trichel' Current Pulses from Negative Needle-to-Plane Coronas," J. Appl. Phys., Vol. 45, pp. 103-113 (1975).

[71] C. Mayoux and M. Goldman, "Partial Discharges in Solid Dielectrics and Corona Discharge Phenomena," J. Appl. Phys., Vol. 44, pp. 3940-3944 (1973).

[72] J. W. Mason and B. Young, "Some Physical Mechanisms Affecting the Distribution of Corona Pulse Intervals", Proc. 7th Int. Conf. on Gas Discharges and Their Applications, Peter Peregrinus, London, pp. 196-199 (1982).

[73] R. E. Vosteen, " DC Electrostatic Voltmeters and Fieldmeters", Conference Record of the 9th Annual Meeting of the IEEE Industry Applications Society, October (1974).

[74] IEEE Standard 1227-1990, "IEEE Guide for the Measurement of DC ElectricField Strength and Ion Related Quantities", IEEE, New York, NY (1990).

[75] A. K. Jonscher and E. F. Owede, "Time and Frequency-resolved Surface Currents on Insulators", IEEE Trans. Elec. Insul., Vol. 25, pp. 1077-1084 (1990).

[76] R. E. Hebner, "Measurement of Electrical Breakdown in Liquids," in The Liquid State and Its Electrical Properties, editors E. E. Kunhardt, L. G. Christophorou, and L. H. Luessen, New York, NY: Plenum Press, pp. 519-537 (1988).

[77] R. E. Tobazeon, "Streamers in Liquids," in The Liquid State and Its Electrical Properties. Edited by E. E. Kunhardt, L. G. Christnphorou, and L. H. Luessen, New York: Plenum Press, pp. 465-500 (1988).

[78] R. Kattan, A. Denat, and O. Lesaint, "Generation, Growth, and Collapse of Vapor Bubbles in Hydrocarbon Liquids under a High Divergent Field," J. Appl. Phys., Vol. 66, pp. 4062-4066 (1989). 
[79] S. M. Korobejnikov and E. F. Yanshin, "Model of Prebreakdown Processes in Liquids under Pulse Voltage," Conference Record, Ninth International Conference on Conduction and Breakdown in Dielectric Liquids, Salford, UK, pp. 398-402 (1.987).

[80] I. Alexeff, M. O. Pace, T. V. Blalock, and A. L. Wintenberg, "Possible Models for the Earliest Prebreakdown Events in dc Stressed Hexane," Conference Record, Tenth International Conference on Conduction and Breakdown in Dielectric Liquids, Grenoble, France, pp. 387-391 (1990).

[31] P. K. Watson and W. G. Chadband, "The Dynamics of Pre-Breakdown Cavities in Viscous Silicone Fluids in Negative Point-Plane Gaps," IEEE Trans. Elect. Ins., Vol. EI-23, pp. 729-738 (1988).

[82] E. F. Kelley, M. Nehmadi, R. E. Hebner, M. O. Pace, A. L. Wintenberg, T. V. Blalock, and J. V. Foust, "Measurement of Partial Discharges in Hexane under dc Voltage," IEEE Trans. Elect. Ins., Vol. EI-24, pp. 1109-1119 (1989).

[83] M. O. Pace, A. L. Wintenberg, T. V. Blalock, E. F. Kelley, G. J. FitzPatrick, C. Fenimore, and H. Yamashita, "Pressure Effects on Partial Discharge in Hexane under dc Voltage," Annual Report, Conference on Electrical Insulation and Dielectric Phenomena, Leesburg, VA; pp. 87-92 (1989).

[84] H. Yamashita, H. Kawai, K. L. Stricklett, and E. F. Kelley, "The Effect of High Pressure on Prebreakdown Phenomena in n-Hexane," Conference Record, Tenth International Conference on Conduction and Breakdown in Dielectric Liquids, Grenoble, France, pp. 404-409 (1990).

[85] K. L. Stricklett, E. F. Kelley, H. Yamashita, C. Fenimore, M. O. Pace, T. V. Blalock, A. L. Wintenberg, and I. Alexeff, "Observation of Partial Discharge in Hexane under High Magnification," Conference Record, Tenth International Conference on Conduction and Breakdown in Dielectric Liquids, Grenoble, France, pp. 381-386 (1990).

[86] E. F. Kelley, "An Image-Preserving Optical Delay for High-Speed Photography," Conference Record, 42nd Annual Conference of the Society for Imaging Science and Technology, Boston, MA, pp. 293-296 (1989).

[87] G. J. FitzPatrick, E. O. Forster, R. E. Hebner, and E. F. Kelley, "Prebreakdown Cathode Processes in Liquid Hydrocarbons," IEEE Trans. Elect. Ins., Vol. EI22, pp. 453-458 (1987).

[88] R. E. Hebner, E. F. Kelley, E. O. Forster, and G. J. FitzPatrick, "Observation of Prebreakdown and Breakdown Phenomena in Liquid Hydrocarbons," J. Electrostatics, Vol. 12, pp. 265-283 (1982). 
[89] R. E. Hebner, E. F. Kelley, G. J. FitzPatrick, and E. O. Forster, "The Effect of Pressure on Streamer Initiation in n-Hexane," Conference Record of the 1986 IEEE International Symposium on Electrical Insulation, Washington, DC, pp. 66-68 (1986).

[90] G. J. FitzPatrick and E. O. Forster, "The Effect of Pressure on the Prebreakdown Events in Toluene," Annual Report, Conference on Electrical Insulation and Dielectric Phenomena, pp. 299-303 (1986).

[91] P. J. McKenny, E. O. Forster, E. F. Kelley, and R. E. Hebner, "Effect of Pressure on the Development of Prebreakdown Streamers," Annual Report, Conference on Electrical Insulation and Dielectric Phenomena, pp. 263-268 (1988).

[92] R. E. Hebner, E. F. Kelley, E. O. Forster, and G. J. FitzPatrick, "Observation of Prebreakdown and Breakdown Phenomena in Liquid Hydrocarbons II. NonUniform Field Conditions," IEEE Trans. Elect. Ins., Vol. EI-20, pp. 281-292 (1985).

[93] G. J. FitzPatrick, P. J. McKenny, and E. O. Forster, "The Effect of Pressure on Streamer Inception and Propagation In Liquid Hydrocarbons," Trans. Elect. Ins., Vol. EI-25, pp. 672-682 (1990).

[94] H. Yamashita and H. Amano, "Pre-Breakdown Current and Light Emission in Transformer Oil," IEEE Trans. Elect. Ins., Vol. EI-20, pp. 247-255 (1985).

[95] H. Yamashita and H. Amano, "Prebreakdown Phenomena in Hydrocarbon Liquids," IEEE Trans. Elect. Ins., Vol. EI-23, pp. 739-750 (1988).

[96] K. C. Kao and J. P. C. McMath, "Time-Dependent Pressure Effect in Liquid Dielectrics," IEEE Trans. Elect. Ins., Vol. EI-5, pp. 64-68 (1970).

[97] J. L. Blue, "B2DE - A Program for Solving Systems of Partial Differential Equations in Two Dimensions," National Bureau of Standards NBSIR-3411 (1986).

[98] A. L. Wintenberg, T. V. Blalock, and M. O. Pace, "High-Bandwidth Measurement of Low-Level Prebreakdown Currents in Liquid Dielectrics," Conference Record of the 1990 IEEE International Symposium on Electrical Insulation, Toronto, Canada, pp. 422-426 (1990).

[99] A. L. Wintenberg, "Development of a Measurement System Optimized for Detecting Prebreakdown Currents in Liquid Dielectrics," Ph.D. Thesis, University of Tennessee, Knoxville, TN, (Dec. 1989).

[100] E. V. Yanshin, K. V. Yanshin, and S. M. Korobejnikov, "Space Charge and Pre-Breakdown Bubble Formation near Point Electrodes under Pulse Voltage," Conference Record, Eighth International Conference on Conduction and Breakdown in Dielectric Liquids, Pavia, Italy, pp. 194-198 (1984). 
[101] E. F. Kelley and R. E. Hebner, "Electro-Optic Field Measurement at a Needle Tip and Streamer Initiation in Nitrobenzene," Annual Report, Conference on Electrical Insulation and Dielectric Phenomena, Claymont, DL, pp. 272-277 (1986).

[102] R. Coelho and J. Debeau, "Properties of the Tip-Plane Configuration," J. Phys. D: Appl. Phys., Vol. 4, pp. 1266-1280 (1971).

[103] International Electrutechnical Commission, High Voltage Test Techniques, IEC Publication 60-1,2,3,4, (1973-1977).

[104] IEEE/ANSI Standard Techniques for High Voltage Testing, IEEE Standard 4, (1978).

[105] Qi Qing-Cheng and W. S. Zaengl, "Investigations of Errors Related to the Measured Virtual Front Time T1 of Lightning Impulses", IEEE Transactions on Power Apparatus and Systems, Vol. PAS-102, pp. 2379-2390 (1983).

[106] Y. X. Zhang, R. H. McKnight, and R. E. Hebner, "Interaction Between Two Dividers Used in Simultaneous Comparison Measurements", IEEE Transactions on Power Delivery, Vol. PD-4, pp. 1586-1594 (1989).

[107] Draft-High Voltage Test Techniques Revision of Publication 60-3 and 60-4; Prepared by WG7 of IEC/TC42 (Feb. 1990).

[108] G. J. FitzPatrick, J. K. Olthoff, E. D. Simmon, and C. P. Fenimore, Jr., "Metrology for Space Power: Metrology Development and Survey of SpaceBased Measurements," NISTIR 4422, (September, 1990), pp. 2-11.

[109] T. R. McComb, R. C. Hughes, H. A. Lightfoot, K. Schon, R. Schulte, R. H. Mcknight and Y. X. Zhang, "International Comparison of HV Impulse Measuring Systems", IEEE Transactions on Power Delivery, Vol. PD-4, pp. 906-915 (1989).

[110] Y. X. Zhang and Y. J. Yang, "The Waveform adjusting of $5400 \mathrm{KV}$ Impulse Generator", High Voltage Engineering, No. 1 (1985).

[111] R. J. Van Brunt, Ed. "Research for Electric Energy Systems-An Annual Report," (1989). 


\begin{tabular}{|c|c|c|}
\hline \multirow{2}{*}{\multicolumn{2}{|c|}{$\begin{array}{l}\text { U.S. DEPARTMENT OF COMMERCE } \\
\text { NATIONAL INSTITUTE OF STARIDARDS AND TECHNOLOGY }\end{array}$}} & $\begin{array}{l}\text { 1. PUELCATION ON AEPOAT NUMEEA } \\
\text { VISTIR } 4691 \\
2 \text { PEAFOAMING OACANIZATION AEPOAT NUMBEA }\end{array}$ \\
\hline & & TION DATE \\
\hline \multicolumn{3}{|c|}{$\begin{array}{l}\text { A. Truamosumirn } \\
\text { Research for Electric Energy Systems - An Annual Reoprt }\end{array}$} \\
\hline \multicolumn{3}{|c|}{$\begin{array}{l}\text { S. ATrHon(s) } \\
\text { William E. And }\end{array}$} \\
\hline \multicolumn{2}{|r|}{ 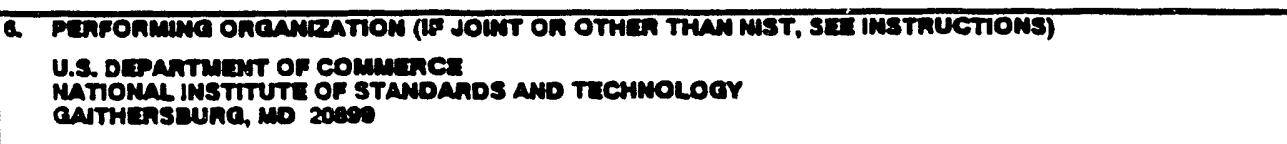 } & 7. CONTRACT/GRANT MUMERA \\
\hline \multicolumn{3}{|c|}{ 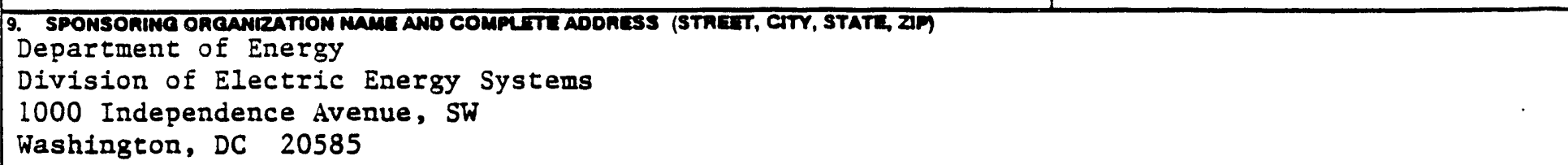 } \\
\hline \multicolumn{3}{|c|}{ 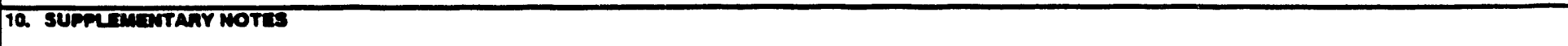 } \\
\hline \multicolumn{3}{|c|}{ 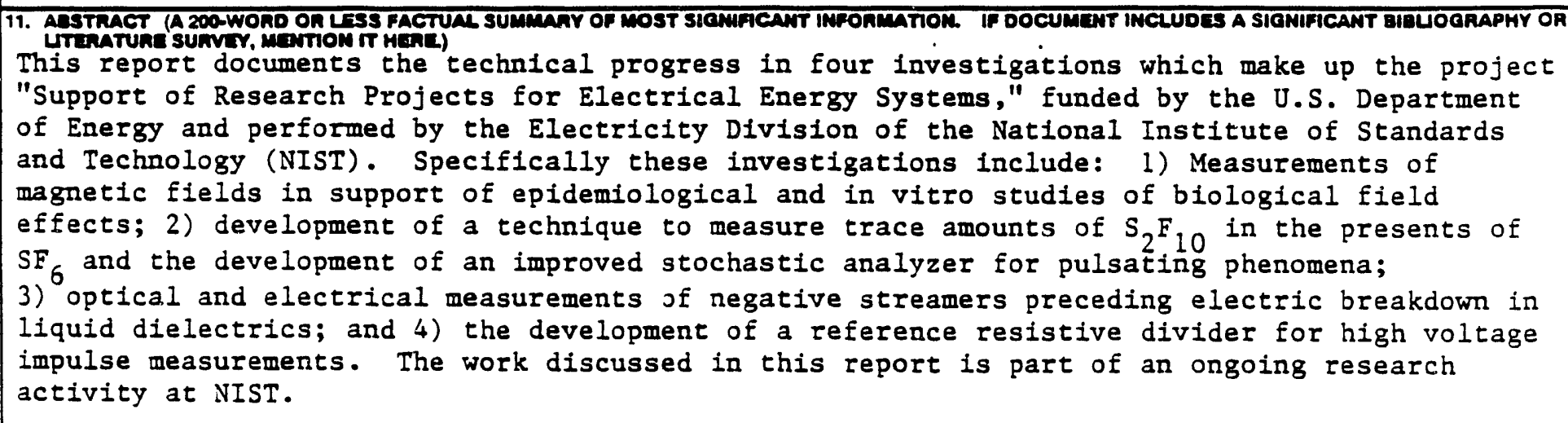 } \\
\hline \multicolumn{3}{|c|}{ 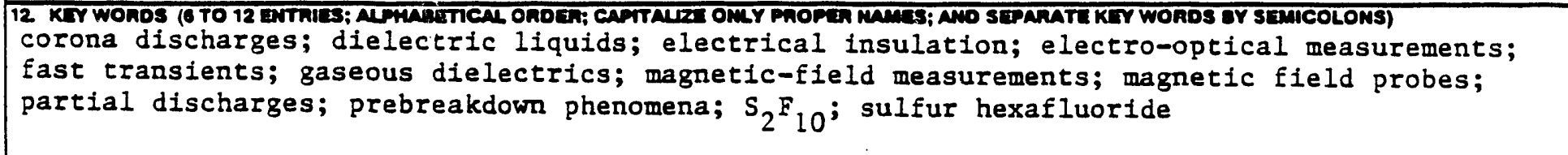 } \\
\hline \multicolumn{3}{|r|}{ 14. NUMBEA OF PAINTED PACES } \\
\hline \multirow[t]{2}{*}{$\mathrm{XX}$} & 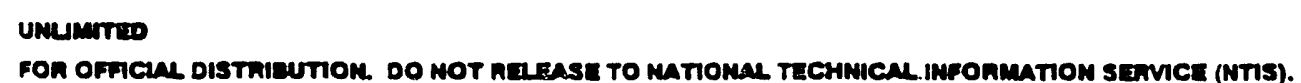 & 105 \\
\hline & 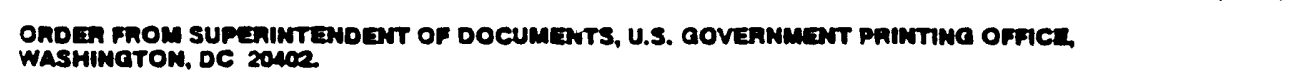 & 15. PAICE \\
\hline $\mathrm{x}$ & 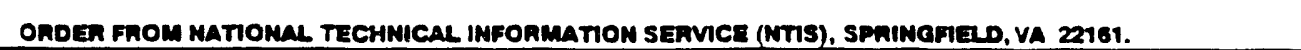 & A06 \\
\hline
\end{tabular}

ELECTRONIC FORM 

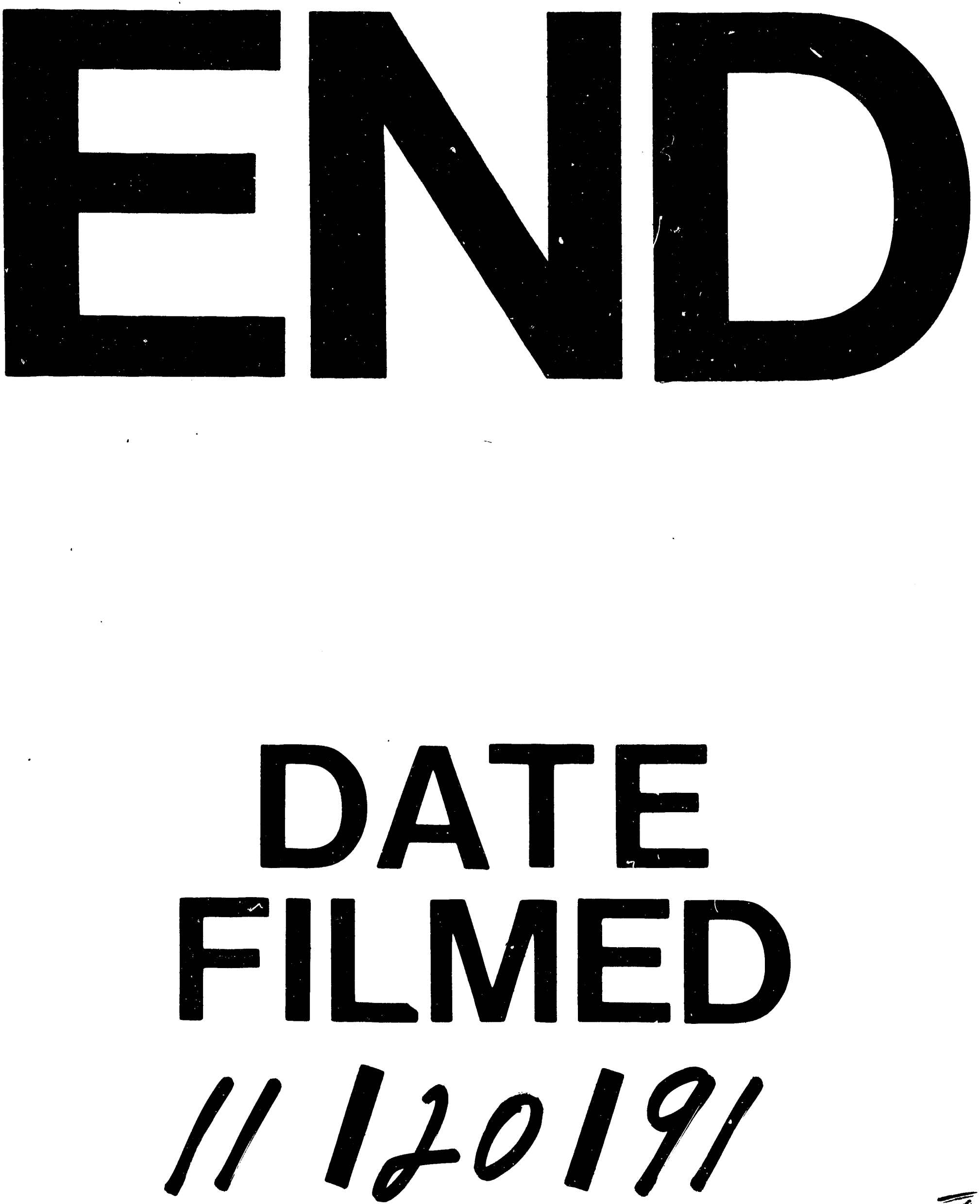

r 
